\title{
Geometric structures on Riemannian manifolds
}

\author{
Naichung Conan Leung
}

\begin{abstract}
In this article, we describe various geometries on Riemannian manifolds via a unified approach using normed division algebras and vector cross products. They include Kähler geometry, Calabi-Yau geometry, hyperkähler geometry, $G_{2}$-geometry and geometry of Riemannian symmetric spaces.
\end{abstract}

\section{Contents}

1. Introduction 162

2. Topology of manifolds 165

2.1. Cohomology and geometry of differential forms 165

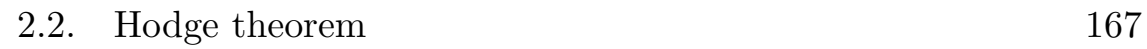

2.3. Witten-Morse theory 171

$\begin{array}{ll}\text { 2.4. Vector bundles and gauge theory } & 172\end{array}$

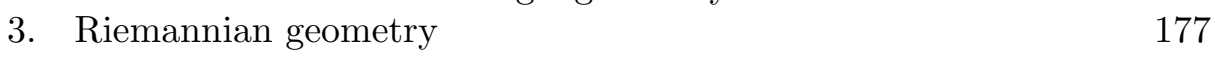

3.1. Torsion and Levi-Civita connections 177

3.2. Classification of Riemannian holonomy groups 178

3.3. Riemannian curvature tensors 178

3.4. Flat tori 180

3.5. Einstein metrics 182

3.6. Minimal submanifolds 183

3.7. Harmonic maps 184

4. Oriented four manifolds 186

4.1. Gauge theory in dimension four 187

4.2. Riemannian geometry in dimension four 188

5. Kähler geometry 190

5.1. Kähler geometry - complex aspects 191

5.2. Kähler geometry—Riemannian aspects 195

5.3. Kähler geometry - symplectic aspects 198

5.4. Gromov-Witten theory 202

(C)2011 International Press 
6. Calabi-Yau geometry 204

6.1. Calabi-Yau manifolds 204

6.2. Special Lagrangian geometry 205

6.3. Mirror symmetry 208

6.4. K3 surfaces 214

7. Calabi-Yau 3-folds 217

7.1. Moduli of CY threefolds 217

7.2. Curves and surfaces in Calabi-Yau threefolds 219

7.3. Donaldson-Thomas bundles over Calabi-Yau threefolds 222

7.4. Special Lagrangian submanifolds in $\mathrm{CY}^{3} \quad 223$

7.5. Mirror symmetry for Calabi-Yau threefolds 223

8. $G_{2}$-geometry 225

8.1. $G_{2}$-manifolds $\quad 225$

8.2. Moduli of $G_{2}$-manifolds 226

8.3. (Co-)associative geometry 227

8.4. $G_{2}$-Donaldson-Thomas bundles 229

8.5. $G_{2}$-dualities, trialities and M-theory 230

9. Geometry of vector cross products 231

9.1. VCP manifolds 232

9.2. Instantons and branes 233

9.3. Symplectic geometry on higher dimensional knot spaces 234

9.4. $\quad \mathbb{C}-$ VCP geometry 235

9.5. Hyperkähler geometry on isotropic knot spaces of CY 236

10. Geometry over normed division algebras 237

10.1. Manifolds over normed algebras 237

10.2. Gauge theory over (special) $\mathbb{A}$-manifolds 240

10.3. A-submanifolds and (special) Lagrangian submanifolds 240

11. Quaternion geometry 242

11.1. Hyperkähler geometry 243

11.2. Quaternionic-Kähler geometry 247

$\begin{array}{ll}\text { 12. Conformal geometry } & 247\end{array}$

13. Geometry of Riemannian symmetric spaces 250

13.1. Riemannian symmetric spaces 250

13.2. Jordan algebras and magic square $\quad 252$

13.3. Hermitian and quaternionic symmetric spaces 255

14. Conclusions 257

$\begin{array}{ll}\text { References } & 258\end{array}$

Acknowledgement: The research of the author is partially supported by a RGC grant from the Hong Kong Government. The author thanks the referee for many useful suggestions for improving the presentation.

\section{Introduction}

Many naturally arising Riemannian manifolds admit rich geometric structures. Projective manifolds in complex algebraic geometry are Kähler 
manifolds. Namely they are Riemannian manifolds with compatible parallel complex structures. Kähler manifolds are also the target spaces for two dimensional $\sigma$-models with $N=2$ supersymmetry (SUSY) in physics.

Using the metric $g$, every orthogonal complex structure $J$ determines a symplectic structure $\omega$ via

$$
\omega(u, v)=g(J u, v)
$$

Symplectic structures are the natural structures on the phase spaces in classical mechanics. Complex geometry studies objects like complex submanifolds and (stable) holomorphic vector bundles and symplectic geometry studies objects likes (special) Lagrangian submanifolds and unitary flat bundles over them. The modern symplectic geometry incorporates holomorphic curves to quantum correct the classical symplectic geometry.

The mirror symmetry conjecture says roughly that the complex geometry and the quantum symplectic geometry can be transformed to each other between any mirror pair of Calabi-Yau manifolds. Calabi-Yau manifolds are Kähler manifolds with $\mathbb{C}$-orientations. Yau proved that every compact Kähler manifold with vanishing first Chern class admits a Calabi-Yau structure. Calabi-Yau manifolds with real dimension six arise in string theory as internal spaces when compactifying ten dimensional spacetimes to our usual spacetime $\mathbb{R}^{3,1}$, i.e. writing ten dimensional spacetimes as products of Calabi-Yau manifolds with $\mathbb{R}^{3,1}$. Similarly, Riemannian manifolds with real dimension 7 (resp. 8) with holonomy $G_{2}$ (resp. Spin (7)) arise in M-theory (resp. F-theory) as internal spaces.

$G_{2}$-manifolds or $\operatorname{Spin}(7)$-manifolds are $\mathbb{O}$-manifolds with or without $\mathbb{O}$-orientations. Recall that the octonion $\mathbb{O}$ is the largest normed division algebra. These two exceptional geometries can also be interpreted as the geometries of 2 -fold or 3 -fold vector cross product (VCP). Note that 1 -fold VCPs are simply orthogonal complex structures. Namely, Kähler manifolds are Riemannian manifolds with parallel 1-fold VCPs. Lagrangian submanifolds and holomorphic curves in Kähler manifolds are branes and instantons on VCP-manifolds. Thus we obtain a unified description of various geometries of calibrated submanifolds, including (co-)associative submanifolds and Cayley submanifolds.

Kähler manifolds with $\mathbb{C}$-VCPs are either Calabi-Yau manifolds or hyperkähler manifolds. For $\mathbb{C}-V C P s$, instantons depend on a phase angle and there are also two types of branes: D-branes and N-branes, corresponding to Dirichlet and Neumann boundary conditions. Again this gives a unified description of the geometries of special Lagrangian submanifolds, complex hypersurfaces and complex Lagrangian submanifolds.

The space of a certain type of geometric structures modulo symmetries is called a moduli space. For manifolds (resp. bundles), the symmetries are diffeomorphisms (resp. gauge transformations). A typical example is the moduli space of complex structures. 
Some important moduli spaces $\mathcal{M}$ have several complex structures and they are hyperkähler manifolds. Studies of moduli spaces had proven to be a very useful approach to understand the underlying geometries. For instance, moduli spaces play essential roles in Donaldson/Seiberg-Witten theory, Gromov-Witten theory and mirror symmetry.

Note that hyperkähler manifolds are $\mathbb{H}$-oriented $\mathbb{H}$-manifolds. Describing various geometries in terms of normed division algebras $\mathbb{A}$ gives us yet another beautiful way to unify many special geometric structures. For instance, when $\mathbb{A}=\mathbb{C}, \mathbb{H}$ and $\mathbb{O}$, (i) $\mathbb{A}$-bundles are holomorphic bundles, Bbundles and Donaldson-Thomas Spin (7)-bundles and (ii) special $\mathbb{A}$-bundles are Hermitian Yang-Mills bundles, hyperholomorphic bundles and Donaldson-Thomas $G_{2}$-bundles. Similarly, (i) $\mathbb{A} / 2$-Lagrangian submanifolds are Lagrangian submanifolds, hyperlagrangian submanifolds and Cayley submanifolds and (ii) special $\mathbb{A} / 2$-Lagrangian submanifolds are special Lagrangian submanifolds, complex Lagrangian submanifolds and (co-)associative submanifolds. Through these unified descriptions, we have established new links among different kinds of geometries and uncovered new results from known facts in other geometries.

As a matter of fact, there are not too many other geometric structures on Riemannian manifolds. Accordingly to the Berger's classification of reduced irreducible Riemannian holonomy groups, they must be one of the following: $S O(n), U(n), S U(n), S p(n) S p(1), S p(n), S p i n(7)$ or $G_{2}$, unless $M$ is a locally symmetric space.

As we will discuss in this survey, these seven possible holonomy groups and their corresponding geometries can be nicely described in terms of normed division algebras (or $\mathbb{A}-\mathrm{VCPs}$ ). Furthermore, using two normed division algebras $\mathbb{A}$ and $\mathbb{B}$, we could also interpret compact Riemannian symmetric spaces as Grassmannians of complex/Lagrangian subspaces in $(\mathbb{A} \otimes \mathbb{B})^{n}$.

In this article, we will first explain the topology and geometry of Riemannian manifolds. Then we explain the complex geometry and the symplectic geometry of Kähler manifolds and their mirror symmetry on Calabi-Yau manifolds. After that, we study the geometry of Calabi-Yau threefolds, $G_{2}$-manifolds and their dualities. After we have familiarized ourselves with these geometries, we give two unified approaches to describe them, namely geometry over normed division algebras and VCP-geometry. In the last section, we explain how Riemannian symmetric spaces can also be understood in a similar fashion by using two normed division algebras.

This unification of all geometries of special holonomy will enhance our understanding of each individual geometry and uncover hidden links among them, as well as among different physical theories. There are several excellent sources on the subject of special holonomy, including [13] [23][24][39][59] $[\mathbf{7 1}][\mathbf{9 5}][\mathbf{1 1 4}]$. Of course, this is a huge subject and the author must apologize that many important topics are inevitably missed in this article. The choices of topics are due to the personal taste and the limited knowledge of the author. The readers should be warned that the list of references is 
unfortunately not incomplete as it is not practical to provide explicit references to all the results mentioned in the paper.

\section{Topology of manifolds}

\subsection{Cohomology and geometry of differential forms.}

Singular and deRham cohomology groups. Inside any given manifold ${ }^{1} M$ of dimension $n$, the most basic cycles are submanifolds $C$. In order to allow $C$ to be singular and to have multiplicities, we sometimes use the parametrized version, for example singular cycles. $k$ dimensional singular cycles modulo homologous equivalences form a finitely generated Abelian group $H_{k}(M, \mathbb{Z})$, the singular homology.

A dual version of this is the singular cohomology $H^{k}(M, \mathbb{Z})$. Modulo torsions, $H^{k}(M, \mathbb{Z})$ equals $\operatorname{Hom}\left(H_{k}(M, \mathbb{Z}), \mathbb{Z}\right)$. There is a cup product

$$
\cup: H^{k}(M, \mathbb{Z}) \otimes H^{l}(M, \mathbb{Z}) \rightarrow H^{k+l}(M, \mathbb{Z}),
$$

giving a ring structure on $H^{*}(M, \mathbb{Z})$. Since $M$ is orientable, we have $H^{n}$ $(M, \mathbb{Z}) \cong \mathbb{Z}$ and Poincaré duality says that $\cup$ is a perfect pairing when $k+l=n$.

The DeRham theorem says that $H^{k}(M, \mathbb{R})$ can be described using differential forms: Let $\Omega^{k}(M)$ be the space of differential forms of degree $k$, i.e. $\Omega^{k}(M)=\Gamma\left(M, \Lambda^{k} T_{M}^{*}\right)$. We consider the deRham complex

$$
0 \rightarrow \Omega^{0}(M) \stackrel{d}{\rightarrow} \Omega^{1}(M) \stackrel{d}{\rightarrow} \cdots \stackrel{d}{\rightarrow} \Omega^{n}(M) \rightarrow 0 \text { with } d^{2}=0,
$$

then

$$
\left.H^{k}(M, \mathbb{R}) \cong \frac{\operatorname{Ker}(d)}{\operatorname{Im}(d)}\right|_{\Omega^{k}(M)} .
$$

Moreover the cup product is simply the wedge product of differential forms and the isomorphism $H^{n}(M, \mathbb{R}) \stackrel{\simeq}{\rightrightarrows} \mathbb{R}$ is given by the integration of the top degree forms.

Volume geometry and symplectic geometry. The existence of a nowhere vanishing $n$-form $v_{M}$ on $M$, called the volume form, is equivalent to the orientability of $M$. Note that the existence of a nowhere vanishing 1-form on $M$ is equivalent to the vanishing of the Euler characteristic, $\chi(M)=0$. For 2 -forms $\omega$, the nowhere vanishing should be replaced by the nondegeneracy, i.e. the map $v \rightarrow \iota_{v} \omega=\omega(v, \cdot)$ gives an isomorphism between $T_{M}$ and $T_{M}^{*}$. This forces $M$ to be of even dimension $n=2 m$ and in this case nondegeneracy is equivalent to the nonvanishing of $\omega^{m} / m$ !, i.e. $\omega^{m} / m$ ! is a volume form on $M$. A nondegenerate two form $\omega$ is called a symplectic form on $M$ if it is also a closed form. Symplectic geometry originated from classical mechanics and now it has become an indispensable part of modern mathematics and physics. For higher degree forms, the natural generalizations of

\footnotetext{
${ }^{1}$ All manifolds are connected compact oriented smooth manifolds unless specified otherwise.
} 
nondegeneracy are either stable forms, as studied by Hitchin [65] or vector cross product forms (section 9 ).

Moser's lemma says that if two volume forms $v_{M}$ and $v_{M}^{\prime}$ have the same volume, i.e. $\int_{M} v_{M}=\int_{M} v_{M}^{\prime}$, then they are the same up to diffeomorphisms. Namely, there exists a diffeomorphism $f \in D i f f^{0}(M)$ satisfying $v_{M}^{\prime}=f^{*} v_{M}$. Thus the moduli space of volume forms on an oriented manifold $M$ is $\mathbb{R} \backslash\{0\}$. If we require the volume forms to be compatible with a fixed orientation on $M$, then the moduli space becomes $\mathbb{R}^{+}$.

Similarly, if two symplectic forms $\omega_{M}$ and $\omega_{M}^{\prime}$ can be connected through symplectic forms representing the same cohomology class in $H^{2}(M, \mathbb{R})$, then $\omega_{M}^{\prime}=f^{*} \omega_{M}$ for some $f \in D i f f^{0}(M)$. Thus the moduli space of symplectic forms on $M$ is locally given by $H^{2}(M, \mathbb{R})$.

The standard volume form (resp. symplectic form) on $\mathbb{R}^{n}$ is $d x^{1} \wedge d x^{2} \wedge$ $\cdots \wedge d x^{n}$ (resp. $\left.d x^{1} \wedge d x^{2}+d x^{3} \wedge d x^{4}+\cdots+d x^{n-1} \wedge d x^{n}\right)$. In fact every volume (resp. symplectic) form on a manifold $M$ is locally standard, the so-called Darboux lemma.

We will denote the group of diffeomorphisms of $M$ preserving a tensor $t$ as $\operatorname{Diff}(M, t)$ and its Lie algebra of vector fields as $\operatorname{Vect}(M, t)$, i.e. $X \in$ $V e c t(M, t)$ if and only if $L_{X} t=0$. Examples of such $t$ 's include volume forms $v$, symplectic forms $\omega$ and metric tensors $g$.

Contracting vector fields with a volume form $v_{M}$ induces an isomorphism between Vect $(M)$ and $\Omega^{n-1}(M)$. By the Cartan formula of the Lie derivative on $\Omega^{*}(M), L_{X}=d \circ \iota_{X}+\iota_{X} \circ d$, we have

$$
L_{X} v_{M}=0 \text { if and only if } d\left(\iota_{X} v_{M}\right)=0 .
$$

Hence

$$
\operatorname{Vect}\left(M, v_{M}\right) \cong \Omega^{n-1}(M) \cap \operatorname{Ker}(d) .
$$

Similarly, for a symplectic form $\omega_{M}$, we have

$$
\operatorname{Vect}\left(M, \omega_{M}\right) \cong \Omega^{1}(M) \cap \operatorname{Ker}(d) .
$$

Given any $m$-dimensional manifold $S$, the total space of the line bundle $\Lambda^{m} T_{S}^{*}$ has a canonical volume form $v_{\text {can }}$. In terms of any local coordinate system $\left(x^{1}, \ldots, x^{m}, y d x^{1} \wedge \cdots \wedge d x^{m}\right)$, we have

$$
v_{\text {can }}=d x^{1} \wedge \cdots \wedge d x^{m} \wedge d y
$$

Similarly, the total space of the cotangent bundle $T_{S}^{*}$ has a canonical symplectic form $\omega_{\text {can }}$. Actually there is a canonical one form $\alpha$ with $\omega_{\text {can }}=$ $-d \alpha$. Concretely, given any point $p=(x, y) \in T^{*} M$ with $y \in T_{x}^{*} M$ and any tangent vector $v$ at $p$ we have $\alpha(v)=y\left(\pi_{*} v\right)$ where $\pi: T^{*} M \rightarrow M$ is the canonical projection $(x, y) \rightarrow x$. In terms of local coordinates $\left(x^{1}, \ldots, x^{m}\right.$, $\left.y_{1} d x^{1}, \ldots, y_{m} d x^{m}\right)$, we have

$$
\begin{gathered}
\alpha=y_{1} d x^{1}+\cdots+y_{m} d x^{m} \text { and } \\
\omega_{\text {can }}=d x^{1} \wedge d y_{1}+\cdots+d x^{m} \wedge d y_{m} .
\end{gathered}
$$


The natural class of submanifolds $S$ in a symplectic manifold $\left(M, \omega_{M}\right)$ consists of Lagrangian submanifolds which are defined as

$$
\operatorname{dim} S=\frac{1}{2} \operatorname{dim} M \quad \text { and }\left.\quad \omega_{M}\right|_{S}=0 .
$$

By an extension of the Darboux lemma, the neighborhood of any Lagrangian submanifold $S \subset\left(M, \omega_{M}\right)$ is symplectomorphic to a neighborhood of $S \subset$ $\left(T_{S}^{*}, \omega_{\text {can }}\right)$. Furthermore, nearby Lagrangian submanifolds are given by graphs of closed one forms on $S$.

To rigidify a symplectic manifold $(M, \omega)$, we choose a compatible metric $g$, i.e. if we define $J: T_{M} \rightarrow T_{M}$ via

$$
g(J u, v)=\omega(u, v),
$$

then $J$ is an orthogonal complex structure on each tangent space. $J$-holomorphic curves $C$ in $M$ are important tools in modern symplectic geometry. For instance, they play important roles in the Gromov-Witten theory (section 5.4) and mirror symmetry (section 6.3). Suppose $\partial C \neq \phi$, then the natural free boundary condition is to require $\partial C \subset L$ for a fixed Lagrangian submanifold $L \subset M$.

Corresponding notions and results for any volume manifold $\left(M, v_{M}\right)$ also hold true and are easier, where Lagrangian submanifolds and $J$-holomorphic curves are replaced by hypersurfaces and domains. Indeed they are parts of the theory of the geometry of vector cross products, including $G_{2}$-geometry.

Given any smooth manifold $\Sigma$, we consider the evaluation map

$$
\begin{gathered}
e v: \Sigma \times \operatorname{Map}(\Sigma, M) \rightarrow M \\
(x, f) \rightarrow f(x) .
\end{gathered}
$$

Suppose $v_{M}$ is a volume form on $M$. By integrating $e v^{*}\left(v_{M}\right)$ over $\Sigma$, we obtain a differential form $\omega_{\mathcal{K}_{\Sigma}(M)}$ on $\operatorname{Map}(\Sigma, M)$ which descends to the space of knots

$$
\mathcal{K}_{\Sigma}(M)=\operatorname{Map}_{\text {emb }}(\Sigma, M) / \operatorname{Diff}(\Sigma) .
$$

When $\Sigma$ has codimension two in $M, \omega_{\mathcal{K}_{\Sigma}(M)}$ is a symplectic form on $\mathcal{K}_{\Sigma}(M)$. We can relate the volume geometry on $M$ with the symplectic geometry on $\mathcal{K}_{\Sigma}(M)$. Such an interpretation is particularly fruitful when we study the geometry of other vector cross products.

2.2. Hodge theorem. A Riemannian metric $g$ on $M$ is a smooth family of positive definite inner products $g_{x}$ on tangent spaces $T_{x} M$. When $M$ is compact and oriented, the Hodge theorem says that we can find a canonical representative for each cohomology class in $H^{k}(M, \mathbb{R})$, namely the form with the smallest norm. Equivalently, $H^{k}(M, \mathbb{R})$ can be identified with the orthogonal complement to $\operatorname{Im}(d)$ inside $\operatorname{Ker}(d)$. That is

$$
\begin{aligned}
H^{k}(M, \mathbb{R}) & =\operatorname{Ker}(d) \cap \operatorname{Ker}\left(d^{*}\right) \\
& =\operatorname{Ker}\left(d d^{*}+d^{*} d\right),
\end{aligned}
$$


where $d^{*}$ is the adjoint to $d$ and $\Delta=d d^{*}+d^{*} d$ is a second order elliptic differential operator, called the Laplacian. Hodge theory says that every deRham cohomology class has a unique representative $\phi$ satisfying $\Delta \phi=0$, called a harmonic form. To better understand the Hodge theorem, we need to introduce a few notions.

Riemannian volume forms. Suppose $M$ is oriented, then there is a consistent way to choose the sign of the square root $\sqrt{\operatorname{det} g_{i j}}$ and define a volume form

$$
d v_{g}=\sqrt{\operatorname{det} g_{i j}} d x^{1} \wedge d x^{2} \wedge \cdots \wedge d x^{n} .
$$

We call it the Riemannian volume form of $(M, g)$. Having a volume form allows us to integrate functions on $M$. In particular $\operatorname{vol}(M)=\int_{M} d v_{g}$ is an important invariant of $(M, g)$. It also allows us to define an inner product on the space of differential forms and other tensors on $M$ as

$$
\|\phi\|^{2}=\int_{M}|\phi(x)|_{g}^{2} d v_{g}
$$

and the Hodge star operator, $*: \Omega^{k}(M) \rightarrow \Omega^{n-k}(M)$ with the defining equation

$$
\phi \wedge * \eta=g(\phi, \eta) d v_{g}
$$

It satisfies $*^{2}=(-1)^{k(n-k)}$.

Let $d^{*}$ be the formal adjoint to $d$ with respect to the above $L^{2}$-inner product $\|\cdot\|$, that is

$$
\int_{M}\langle d \phi, \eta\rangle_{\Lambda^{k+1} T^{*}}=\int_{M}\left\langle\phi, d^{*} \eta\right\rangle_{\Lambda^{k} T^{*}}
$$

for any $\phi \in \Omega^{k}(M)$ and $\eta \in \Omega^{k+1}(M)$. We have suppressed the volume form $d v_{g}$ and we will continue to do this. Using $\|\cdot\|$ on $\operatorname{Ker}(d) \subset \Omega^{k}(M)$, one expects to be able to split the exact sequence

$$
0 \rightarrow \operatorname{Im}(d) \rightarrow \operatorname{Ker}(d) \rightarrow H^{k}(M) \rightarrow 0
$$

and get

$$
\begin{aligned}
H^{k}(M) & \cong \operatorname{Ker}(d) \cap(\operatorname{Im} d)^{\perp} \\
& =\operatorname{Ker}(d) \cap \operatorname{Ker}\left(d^{*}\right) \subset \Omega^{k}(M) .
\end{aligned}
$$

By direct computations, we have

$$
d^{*}=(-1)^{n k+1} * d *: \Omega^{k+1}(M) \rightarrow \Omega^{k}(M) .
$$

We define the second order self-adjoint differential operator Laplacian as follows,

$$
\Delta=\left(d+d^{*}\right)^{2}=d d^{*}+d^{*} d: \Omega^{k}(M) \rightarrow \Omega^{k}(M) .
$$

This operator is elliptic, i.e its symbol is an invertible algebraic operator. Ellipticity is an important notion, for instance its eigenspaces are of finite dimension and the eigenspace decomposition holds on a suititable Hilbert space completion. 
For instance, $\Delta=-\sum_{j}\left(\frac{\partial}{\partial x^{j}}\right)^{2}$ on $\mathbb{R}^{n}$ and for functions on a general manifold, we have

$$
\Delta f=\frac{-1}{\sqrt{g}} \frac{\partial}{\partial x^{i}}\left(\sqrt{g} g^{i j} \frac{\partial f}{\partial x^{j}}\right)
$$

where $g=\operatorname{det} g_{i j}$.

It is clear that

$$
\int_{M}\langle\Delta \phi, \phi\rangle=\int_{M}|d \phi|^{2}+\int_{M}\left|d^{*} \phi\right|^{2} .
$$

This implies that $\Delta$ is a self-adjoint (i.e. $\Delta=\Delta^{*}$ ) non-negative operator and

$$
\operatorname{Ker}(\Delta)=\operatorname{Ker}(d) \cap \operatorname{Ker}\left(d^{*}\right) .
$$

The harmonic equation $\Delta \phi=0$ is also the Euler-Lagrange equation for minimizing $\int|\phi|^{2}$ for closed forms representing a fixed cohomology class.

When $M=S^{1}=\mathbb{R} / 2 \pi \mathbb{Z}$, we have $\Delta=-\frac{d^{2}}{d \theta^{2}}$. The Fourier series expresses any smooth function $f(\theta)=f(\theta+2 \pi)$ on $S^{1}$ as

$$
f(\theta)=\sum_{\nu \in \mathbb{Z}} \hat{f}(\nu) e^{i \nu \theta} .
$$

Note that $e^{i \nu \theta}$ is an eigenfunction of $\Delta$ with eigenvalue $\nu^{2}$. The Hodge theorem is a generalization of the Fourier series on $S^{1}$ to any compact oriented Riemannian manifold.

The Hodge theorem says that $\Delta$ has an eigenvalue decomposition similar to a symmetric matrix. More precisely (i) each eigenspace

$$
\Omega_{\lambda}^{k}(M):=\left\{\phi \in \Omega^{k}(M): \Delta \phi=\lambda \phi\right\}
$$

is finite dimensional; (ii) If we denote $\operatorname{Spec}(M)=\left\{\lambda \in \mathbb{R}: \Omega_{\lambda}^{k}(M) \neq 0\right\}=$ $\left\{\lambda_{0}<\lambda_{1}<\cdots\right\} \subset \mathbb{R}_{\geq 0}$, the spectrum of $\Delta$, then $\operatorname{Spec}(M)$ is a unbounded set and without any accumulation point; (iii) Every $\phi \in \Omega^{k}(M)$ can be expressed as

$$
\phi=\sum_{i=0}^{\infty} \phi_{i} \quad \text { with } \phi_{i} \in \Omega_{\lambda_{i}}^{k}
$$

uniquely as a convergent power series in $L_{1}^{2}$-norm. That is

$$
\Omega^{k}(M)_{L_{1}^{2}}=\overline{\bigoplus_{i=1}^{\infty} \Omega_{\lambda_{i}}^{k}(M) .}
$$

The Hodge theorem immediately implies that the dimension of $H^{k}(M)$ is finite. Since $*: \Omega^{k}(M) \rightarrow \Omega^{n-k}(M)$ preserves harmonicity, $*$ induces an isomorphism between $H^{k}(M)$ with $H^{n-k}(M)$. This gives the Poincaré duality. However, the wedge product of harmonic forms is usually not harmonic (unless one of them is parallel). 
Existence of harmonic representatives for cohomology classes has many important applications. For instance, using the Bochner-Weitzenbock formula, one shows that harmonic one forms on manifolds with positive Ricci curvature must vanish and therefore $H^{1}(M)=0$ by the Hodge theorem.

The Hodge theorem applies equally well to any elliptic complex besides the deRham complex. These include the deRham complex coupled with a flat unitary connection; the Dolbeault complex of $\bar{\partial}$-operator for any complex manifold which can also be coupled with a holomorphic vector bundle and similar elliptic complexes for manifolds with other special geometric structures.

The eigenvalue decomposition of $\Delta$ on $\Omega^{k}(M)$ is compatible with the deRham complex in the sense that $d\left(\Omega_{\lambda}^{k}\right) \subset \Omega_{\lambda}^{k+1}$. Hence, for any $\lambda$, we have a finite dimensional complex,

$$
0 \rightarrow \Omega_{\lambda}^{0}(M) \stackrel{d}{\rightarrow} \Omega_{\lambda}^{1}(M) \stackrel{d}{\rightarrow} \cdots \stackrel{d}{\rightarrow} \Omega_{\lambda}^{n}(M) \rightarrow 0,
$$

When $\lambda=0$ this complex is trivial, i.e. $d=0$, and otherwise it is an exact complex, i.e.

$$
\left.(\operatorname{Ker}(d) / \operatorname{Im}(d))\right|_{\Omega_{\lambda}^{*}}=0 \text { for } \lambda \neq 0
$$

In particular,

$$
\left.\left.H^{k}(M) \cong \frac{\operatorname{Ker}(d)}{\operatorname{Im}(d)}\right|_{\Omega^{k}(M)} \cong \Omega_{0}^{k}(M) \cong \operatorname{Ker}(\Delta)\right|_{\Omega^{k}(M)}
$$

The exactness of $\left(\Omega_{\lambda}^{*}, d\right)$ implies that $\sum_{k}(-1)^{k} \operatorname{dim} \Omega_{\lambda}^{k}=0$ when $\lambda \neq 0$. Thus

$$
\begin{aligned}
\chi(M) & :=\sum_{k}(-1)^{k} \operatorname{dim} H^{k}(M)=\sum_{k}(-1)^{k}\left(\sum_{\lambda \geq 0} e^{-t \lambda} \operatorname{dim} \Omega_{\lambda}^{k}(M)\right) \\
& =\sum_{\lambda \geq 0} e^{-t \lambda}\left(\sum_{k}(-1)^{k} \operatorname{dim} \Omega_{\lambda}^{k}(M)\right) .
\end{aligned}
$$

Note that the factors $e^{-t \lambda}$ 's are inserted in order to ensure the convergence of the RHS for $t>0$ when we interchange the order of summations. By formally letting $t$ goes to zero, the Euler characteristic $\chi(M)$ can be naturally interpreted as $\sum_{k}(-1)^{k} \operatorname{dim} \Omega^{k}(M)$, the super-dimension of the space of differential forms.

It is interesting to note that for any given $k, \operatorname{dim} \Omega_{\lambda}^{k}(M)$ is the multiplicity of the eigenvalue $e^{-t \lambda}$ for the heat operator $e^{-t \Delta}$ on $\Omega^{k}(M)$. Hence,

$$
\begin{aligned}
\chi(M) & =\sum_{k}(-1)^{k}\left(\sum_{\lambda \geq 0} e^{-t \lambda} \operatorname{dim} \Omega_{\lambda}^{k}(M)\right) \\
& =\sum_{k}(-1)^{k} \operatorname{Tr}_{\Omega^{k}} e^{-t \Delta} .
\end{aligned}
$$


The heat operator $e^{-t \Delta}$ has a nice asymptotic expansion as $t \rightarrow 0^{+}$. Applying some clever tricks, which are motivated from supersymmetry in physics, we can express $\chi(M)$ in terms of the curvature of $M$, or characteristic classes. The above arguments are particularly useful in studying the index problem for first order elliptic complexes and give a short proof [5] for the AtiyahSinger index theorem $[6]$.

2.3. Witten-Morse theory. Suppose $f: M \rightarrow \mathbb{R}$ is a smooth function such that its Hessian $\nabla^{2} f$ is non-degenerate at every critical point. Such an $f$ is called a Morse function. Witten [131] studied a twisted version of the deRham complex $d_{t}:=e^{-t f} d e^{t f}$ using $f$ for any $t \in \mathbb{R}$,

$$
0 \rightarrow \Omega^{0}(M) \stackrel{d_{t}}{\rightarrow} \Omega^{1}(M) \stackrel{d_{t}}{\rightarrow} \cdots \stackrel{d_{t}}{\rightarrow} \Omega^{n}(M) \rightarrow 0 \quad \text { with } d_{t}^{2}=0 .
$$

One can regard this as a non-unitary gauge transformation of the trivial connection on $M \times \mathbb{C}$ and hence it defines the same cohomology group, i.e.

$$
\left.H^{k}(M) \cong \frac{\operatorname{Ker}\left(d_{t}\right)}{\operatorname{Im}\left(d_{t}\right)}\right|_{\Omega^{k}(M)}
$$

Notice that the adjoint of $d_{t}$ is $d_{t}^{*}=e^{t f} d^{*} e^{-t f}$ and

$$
\begin{aligned}
\Delta_{t} & =\left(d_{t}+d_{t}^{*}\right)^{2} \\
& =\Delta+t^{2}|d f|^{2}+t \nabla^{2} f .
\end{aligned}
$$

Here the action of the Hessian $\nabla^{2} f$ on differential forms is given by a combination of interior and exterior multiplications $\left.\left(\nabla^{2} f\right)_{i j} g^{i k}\left(\frac{\partial}{\partial x^{k}}\right\lrcorner\right)\left(d x^{j} \wedge\right)$.

As $t$ becomes large, the quadratic term in $t$ plays the dominant role. Through semi-classical analysis, one shows that (i) small eigenvalues of $\Delta_{t}$ are in one-to-one correspondence with critical points of $f$ and (ii) all other eigenvalues go to infinity.

Recall that the cohomology groups can be computed by a truncated finite dimensional complex $\left(\Omega_{\leq C}^{*}=\bigoplus_{\lambda \leq C} \Omega_{\lambda}^{*}, d_{t}\right)$ for any positive cutoff $C$ for eigenvalues. As $t$ becomes large, one show that

$$
\Omega_{\leq C}^{k} \cong \bigoplus_{\begin{array}{c}
d f(p)=0 \\
\operatorname{index}(p)=k
\end{array}}^{\bigoplus} \mathbb{R}\langle p\rangle \text { and } \quad d_{t}\langle p\rangle=\sum_{\begin{array}{c}
d f(q)=0 \\
\operatorname{index}(q)=k+1
\end{array}} n(p, q)\langle q\rangle
$$

where $n(p, q)$ is the (algebraic) number of gradient flow lines of $f$ joining $p$ to $q$. Thus $H^{*}(M)$ can be computed by counting gradient flow lines between critical points. Therefore Morse theory is just Hodge theory twisted by $f$ !

We remark that the Witten-Morse theory is particularly important in studying the middle dimensional topology of various infinite dimensional function spaces. In these cases, the index of any critical point is infinite but their differences are finite. Examples include the Floer theory for the ChernSimons functional on the space of connections over three manifolds (section 2.4); the Floer theory for the symplectic area functional on the loop spaces 
of symplectic manifolds (section 5.4) and so on. These structures are parts of topological field theories when we include appropriate boundary theories.

2.4. Vector bundles and gauge theory. A rank $r$ vector bundle over $M$ is a smooth family of $r$ dimensional vector spaces $E_{x}$ parametrized by $x \in M$. We write

$$
\mathbb{R}^{r} \rightarrow E \stackrel{\pi}{\rightarrow} M
$$

with $E_{x}=\pi^{-1}(x)$. The tangent bundle $T_{M}$ is such an example. We can also consider complex vector bundles when each $E_{x}$ is a complex vector space. By gluing local trivializations of $E$ using a partition of unity, $E$ can always be embedded as a subbundle of a trivial bundle $M \times \mathbb{C}^{N}$. The quotient bundle of the injective homomorphism $E \rightarrow M \times \mathbb{C}^{N}$ could be regarded as the negative of $E$. The construction of integers from natural numbers can be generalized here to give virtual bundles $\left[E_{1}-E_{2}\right]$ and they generate an Abelian group called the topological $K$-theory $K(M)$ of $M$.

Given any complex vector bundle $E$, one can construct cohomology classes which are functorial topological invariants of $E$. Examples includes Chern classes $c(E)$ and the Chern characters $c h(E)$. Furthermore,

$$
\operatorname{ch}: K(M) \rightarrow \bigoplus_{k=0} H^{2 k}(M, \mathbb{Q})
$$

is a ring homomorphism. Modulo torsions, it is an isomorphism. These characteristic classes (modulo torsions) can be described using differential forms via the Chern-Weil theory as described below.

Connections, curvature and Chern classes. First we need to define connections or covariant derivatives, which are first order differential operators $D_{A}$ on $E$-valued differential forms,

$$
D_{A}: \Omega^{0}(M, E) \rightarrow \Omega^{1}(M, E),
$$

satisfying

$$
D_{A}(f s)=d f \otimes s+f \cdot D_{A} s,
$$

where $f$ (resp. $s$ ) is any function on $M$ (resp. section of $E$ ). We can extend $D_{A}$ to $\Omega^{k}(M, E)$ by coupling it with $d$. A section $s$ is called parallel, or a covariant constant, if $D_{A} s=0$. Locally, $E$ is a trivial bundle and $D_{A}=$ $d+A$ for some $A \in \Omega^{1}(M, E n d(E))$. There are plenty of connections and the difference of any two is an element in $\Omega^{1}(M, E n d(E))$. Since $d^{2}=0$,

$$
\left(D_{A}\right)^{2}: \Omega^{0}(M, E) \rightarrow \Omega^{2}(M, E)
$$

can be shown to be a zeroth order operator. Namely there exists $F_{A} \in$ $\Omega^{2}(M$, End $(E))$, called the curvature tensor satisfying

$$
\left(D_{A}\right)^{2} s=F_{A} s
$$

for any section $s$. Bianchi identity says that

$$
D_{A} F_{A}=0 \in \Omega^{3}(M, E n d(E)) .
$$


This implies that

$$
\frac{1}{k !} \operatorname{Tr}\left(\frac{i}{2 \pi} F_{A}\right)^{k} \in \Omega^{2 k}(M, \mathbb{R})
$$

is a closed differential form for any $k$. The cohomology class it represents can be shown to be the $k^{t h}$ Chern character $c h_{k}(E) \in H^{2 k}(M, \mathbb{Q})$. To put them together, we write

$$
\begin{aligned}
\operatorname{ch}(E) & =r+\sum_{k=1}^{n} c h_{k}(E) \\
& =\left[\exp \left(\frac{i}{2 \pi} F_{A}\right)\right]
\end{aligned}
$$

where $r$ is the rank of $E$. Similarly the Chern class of $E$ can be defined as

$$
\begin{aligned}
c(E)_{\mathbb{R}} & =1+c_{1}(E)_{\mathbb{R}}+\cdots+c_{r}(E)_{\mathbb{R}} \\
& =\left[\operatorname{det}\left(I_{E}+\frac{i}{2 \pi} F_{A}\right)\right] \in \bigoplus_{k=0} H^{2 k}(M, \mathbb{R}) .
\end{aligned}
$$

In particular $c_{1}(E)_{\mathbb{R}}=c h_{1}(E)=\left[\frac{i}{2 \pi} F_{A}\right]$.

Geometrically, a connection $D_{A}$ identifies any two fibers $E_{x}, E_{x^{\prime}}$ along any path $\gamma(t)$ joining $x$ and $x^{\prime}$ in $M$. This is done by extending any base $s_{1}(x), \ldots, s_{r}(x)$ of $E_{x}$ to the whole path by solving the ODE $D_{\dot{\gamma}(t)} s_{j}(\gamma(t))=$ 0 . This identification is called the parallel transport along $\gamma$. If we choose a polar coordinates around a given point $x_{0} \in M$ and use parallel transports along radial paths to identify $E_{x}$ with $E_{x_{0}}$ for every $x$ in a small neighborhood $U$ of $x_{0}$, then we obtain a local trivialization

$$
\left.E\right|_{U} \cong U \times \mathbb{C}^{r}
$$

With respect to this trivialization, $D_{A}$ coincides with the trivial connection on $U \times \mathbb{C}^{r}$ up to first order at $x_{0}$, that is

$$
D_{A}=d+O\left(|x|^{2}\right)
$$

Indeed the coefficients of the second order terms are precisely the curvature tensor of $D_{A}$ at the point $x_{0}$.

Holonomy and flat connections. The parallel transport along any closed loop based at $x_{0} \in M$ defines an element in $G L\left(E_{x_{0}}\right)$, called the holonomy $h l_{D_{A}}(\gamma)$ of $D_{A}$ around $\gamma$. This gives a map on the based loop space of $M$,

$$
h_{D_{A}}: \Omega_{x_{0}}(M) \rightarrow G L\left(E_{x_{0}}\right) .
$$

The image of $h o l_{D_{A}}$ in $G L(r, \mathbb{C})$ is independent of $x_{0} \in M$, up to conjugations, called the holonomy group of $D_{A}$. Roughly speaking, curvature is the holonomy for infinitesimal loops. In particular, when the curvature is zero, $\operatorname{hol}_{D_{A}}(\gamma)$ equals the identity for any contractible loop. That is $\operatorname{hol}_{D_{A}}(\gamma)$ depends only on the homotopy class of $\gamma$, thus we obtain a homomorphism

$$
\operatorname{hol}_{D_{A}}: \pi_{1}\left(M, x_{0}\right) \rightarrow G L\left(E_{x_{0}}\right) .
$$


Up to conjugacy, this map is independent of the base point $x_{0} \in M$. Moreover, if we conjugate $D_{A}$ by an automorphism $g \in A u t(E)=\mathcal{G}_{E}$, also called a gauge transformation, $g \cdot D_{A}=g^{-1} \circ D_{A} \circ g$, we have

$$
F_{g \cdot A}=g^{-1} F_{A} g=0 .
$$

Moreover, $D_{A}$ and $g \cdot D_{A}$ give the same holonomy map up to conjugation. Thus we have a natural identification

$$
\left\{D_{A} \mid F_{A}=0\right\} / \mathcal{G} \stackrel{\text { hol }}{\longrightarrow} \operatorname{Hom}\left(\pi_{1}(M), G L(r, \mathbb{C})\right) / A d(G L(r, \mathbb{C})) .
$$

Indeed the universal covering

$$
\pi_{1}(M) \rightarrow \tilde{M} \rightarrow M
$$

is the universal flat connection is the sense that given any flat connection $D_{A}$ over $M$ with

$$
\rho: \pi_{1}(M) \rightarrow G L(r, \mathbb{C})
$$

the corresponding holonomy map, then the trivial connection $d$ on $\tilde{M} \times \mathbb{C}^{r}$ over $\tilde{M}$ descends to the $\mathbb{C}^{r}$-bundle $\tilde{M} \times{ }_{\rho} \mathbb{C}^{r}$ over $M$ and becomes $D_{A}$, up to gauge symmetries.

The curvature of $D_{A}$ being zero is equivalent to the following sequence

$$
0 \rightarrow \Omega^{0}(M, E) \stackrel{D_{A}}{\rightarrow} \Omega^{1}(M, E) \stackrel{D_{A}}{\rightarrow} \cdots \stackrel{D_{A}}{\rightarrow} \Omega^{n}(M, E) \rightarrow 0
$$

being a complex, that is $\left(D_{A}\right)^{2}=0$. Its cohomology group

$$
H_{D_{A}}^{k}(M, E)=\left.\frac{\operatorname{Ker}\left(D_{A}\right)}{\operatorname{Im}\left(D_{A}\right)}\right|_{\Omega^{k}(M, E)}
$$

generalizes the deRham cohomology group.

Cohomology groups of low degrees have important geometric significances. If we denote the symmetry group of $D_{A}$ as

$$
\operatorname{Aut}\left(E, D_{A}\right)=\left\{g \in \operatorname{Aut}(E): g \cdot D_{A}=D_{A}\right\},
$$

then its Lie algebra equals $H_{D_{A}}^{0}(M, E n d(E))$. Moreover, the tangent space of a smooth point $D_{A}$ in the moduli space $\mathcal{M}^{\text {flat }}(M)$ of flat connections on $E$ equals $H_{D_{A}}^{1}(M, E n d(E))$. This is because if $D_{A}+t B$ is a flat connection for all $B$, the flatness $\left(D_{A}+t B\right)^{2}=0$ is equivalent to

$$
D_{A} B+t[B, B]=0 .
$$

Modulo $t$, we have $D_{A} B=0$, i.e. $[B] \in H_{D_{A}}^{1}(M, E n d(E))$. By similar reasonings, we have the obstructions of smoothness of $\mathcal{M}^{\text {flat }}(M)$ lie inside $H_{D_{A}}^{2}(M, \operatorname{End}(E))$.

In order to use the Hodge theorem to represent elements in $H^{k}(M, E)$ by harmonic forms, we need to choose a metric $g$ on $M$ and also a Hermitian 
metric $h$ on $E$ in such a way that $D_{A} h=0$. As in the manifold case, we have

$$
H_{D_{A}}^{k}(M, E)=\left.\operatorname{Ker}\left(D_{A} D_{A}^{*}+D_{A}^{*} D_{A}\right)\right|_{\Omega^{k}(M, E)}
$$

where $D_{A}^{*}$ is the formal adjoint of $D_{A}$.

Geometrically, $D_{A} h=0$ means that parallel transports by $D_{A}$ preserve the inner product $h\left(s_{1}(x), s_{2}(x)\right)$. Similarly, if we treat our complex vector bundle $E$ as a real vector bundle equipped with a real endomorphism $J: E \rightarrow$ $E$ satisfying $J^{2}=-I_{E}$, then $D_{A}$ being a connection of the complex bundle is equivalent to one on a real bundle which satisfies $D_{A} J=0$. We could also consider other fiberwise structures on $E$ and connections that preserve them in a similar fashion. Their curvatures will then preserve these structures infinitesimally. For example, for a complex (resp. Hermitian) connection, its curvature lies inside $\Omega^{2}\left(M, g l_{\mathbb{C}}(E)\right)$ (resp. $\left.\Omega^{2}(M, u(E))\right)$. In general we write $F_{A} \in \Omega^{2}(M, a d(E))$.

When $E$ is a real vector bundle with a metric $g$, for instance the tangent bundle of a Riemannian manifold, then the curvature of any orthogonal connection lies inside

$$
F_{A} \in \Omega^{2}(M, a d(E)) \cong \Omega^{2}\left(M, \Lambda^{2} E^{*}\right)
$$

because $s o(r) \simeq \Lambda^{2} \mathbb{R}^{r *}$.

Yang-Mills connections. When $M$ is an oriented Riemannian manifold, we can measure the length $\left|F_{A}\right|$ and define the Yang-Mills energy functional,

$$
\begin{gathered}
Y M: \mathcal{A}(E, h) / \mathcal{G}_{E} \rightarrow \mathbb{R} \\
Y M\left(D_{A}\right)=\frac{1}{2} \int_{M}\left|F_{A}\right|^{2} d v_{g} .
\end{gathered}
$$

One looks for connections which minimize $Y M$. The Euler-Lagrange equation for critical points of the Yang-Mills functional is given by the following Yang-Mills equation

$$
D_{A}^{*} F_{A}=0 \in \Omega^{1}(M, a d(E)) .
$$

This second order system of differential equations in $D_{A}$ is elliptic modulo gauge symmetries. We can usually reduce it to an easier first order equation when $M$ admits special geometric structures. We will see many such examples in this article.

Certainly flat connections are absolute minima for the Yang-Mills functional. But this is usually an over-determined system of equations unless $\operatorname{dim}_{\mathbb{R}} M \leq 3$. When $\operatorname{dim}_{\mathbb{R}} M=3$, Casson found a way to count the number of flat $S U$ (2)-connections on any homology three spheres and defined the Casson invariant. In this dimension, flat connections are critical points of the Chern-Simons functional: For any connection $D_{A}=d+A$ on a topologically trivial bundle,

$$
C S\left(D_{A}\right)=\int_{M} \operatorname{Tr}\left(A \wedge d A+\frac{2}{3} A \wedge A \wedge A\right) .
$$


This is invariant under gauge transformations in the identity component of $\mathcal{G}_{E}$. Thus $C S$ is only a multi-valued function on $\mathcal{A}(E) / \mathcal{G}_{E}$. A more intrinsic way to define $C S$ is to write $M$ as the boundary $M=\partial Z$ of a four manifold $Z$ and extend $D_{A}$ over $Z$, then

$$
C S\left(D_{A}\right)=\int_{Z} \operatorname{Tr}\left(F_{A}\right)^{2} .
$$

Floer $[\mathbf{4 2}]$ and others developed the infinite dimensional Witten-Morse theory for $C S$ on $\mathcal{A}(E) / \mathcal{G}_{E}$ and defined the Floer cohomology $H F_{C S}(M)$ for three manifolds $M$ whose Euler characteristic is the Casson invariant. Roughly speaking, $H F_{C S}(M) \cong H^{\infty / 2}\left(\mathcal{A}(E) / \mathcal{G}_{E}\right)$ and it measures the middle dimensional topology of $\mathcal{A}(E) / \mathcal{G}_{E}[\mathbf{3}]$.

When $M$ is the boundary of a four dimensional manifold $Z_{1}$, then the Donaldson polynomial invariants (see section 4.1) for differentiable structures on closed four manifolds has a natural generalization for $Z_{1}$ which takes values in $H F_{C S}(M)$ and are called the relative Donaldson invariants. Various gluing formulas allow us to compute the Donaldson invariants for a closed four manifold $Z$ in terms of relative Donaldson invariants for $\left(Z_{i}, M\right)$ 's under connected sum decomposition $Z=Z_{1} \#_{M} Z_{2}$. This defines a three dimensional topological field theory $(T F T)$.

We can further decompose the three manifold $M=M_{1} \#_{\Sigma} M_{2}$ into a connected sum along a Riemann surface $\Sigma$ of genus $g$. It is called a handlebody decomposition if $M$ is a (rational) homology 3-sphere and $\operatorname{dim} H^{1}\left(M_{i}\right)=g$, i.e. half of $H^{1}(\Sigma)$ vanish on $M_{1}$ and the other half vanish on $M_{2}$. The moduli space $\mathcal{M}_{\Sigma}^{\text {flat }}$ of flat $S U(2)$-connections on $\Sigma$ is a symplectic manifold of dimension $6 g-6$ and each $\mathcal{M}_{M_{i}}^{\text {flat }}$ is a Lagrangian submanifold in $\mathcal{M}_{\Sigma}^{\text {flat }}$. The Lagrangian intersection $\mathcal{M}_{M_{1}}^{\text {flat }} \cap \mathcal{M}_{M_{2}}^{\text {flat }}$ is precisely the discrete set of flat connections on the closed three manifold $M$. Thus Atiyah [3] conjectured that $H F_{C S}(M)$ can be identified with another Floer cohomology $H F_{L a g r}^{\mathcal{M}_{\Sigma}^{\text {flat }}}\left(\mathcal{M}_{M_{1}}^{\text {flat }}, \mathcal{M}_{M_{2}}^{\text {flat }}\right)$ for the Lagrangian intersection theory (section 5.4).

Witten [133] considered the path integral of Chern-Simons functional over $\mathcal{A}(E, h) / \mathcal{G}_{E}$ to give a geometric interpretation of the Jones polynomial of knots and also defined new invariants for three manifolds.

A complexified version of this Chern-Simons theory for Calabi-Yau threefolds, and also for $G_{2}$-manifolds, plays an important role in mirror symmetry $[40][91]$.

Second fundamental forms. We remark that if $E=E_{1} \oplus E_{2}$ is a direct sum of vector bundles and we write any connection $D$ on $E$ accordingly

$$
D=\left(\begin{array}{cc}
D_{1} & B_{21} \\
B_{12} & D_{2}
\end{array}\right)
$$

then $D_{i}$ is a connection on $E_{i}$ and we called $B_{21} \in \Omega^{1}\left(M, \operatorname{Hom}\left(E_{2}, E_{1}\right)\right)$ and $B_{12} \in \Omega^{1}\left(M, H o m\left(E_{1}, E_{2}\right)\right)$ the second fundamental forms of $D$. 
If $D$ is an orthogonal connection with respect to some inner product $h$ on $E$, then any subbundle $E_{1}$ in $E$ determine such a decomposition with $E_{2}$ being the orthogonal complement to $E_{1}$ and $B_{21}=-B_{12} *$.

\section{Riemannian geometry}

3.1. Torsion and Levi-Civita connections. Suppose $M$ is a submanifold in $\mathbb{R}^{N}$, the restriction of the Euclidean metric is a Riemannian metric $g$ on $M$. Moreover the usual differential is a trivial connection $d$ on $\mathbb{R}^{N}$ and it restricts to an orthogonal connection on $(M, g)$.

Given any metric $g$ on $M$, such an isometric embedding always exists by the celebrated theorem of Nash. There could be many isometric embeddings of a given $(M, g)$ into Euclidean spaces, but the induced orthogonal connections are always the same. It can be characterized intrinsically as the unique connection $\nabla$ on $T_{M}$ satisfying $\nabla g=0$ and $\operatorname{Tor}(\nabla)=0$, called the Levi-Civita connection of $(M, g)$.

Let us briefly explain the torsion tensor Tor $(\nabla)$ for any connection on the tangent bundle $T_{M}$. Connections on $T_{M}$ are called affine connections. Each $\nabla$ induces a dual connection on $T_{M}^{*}$,

$$
\nabla: \Omega^{0}\left(M, T_{M}^{*}\right) \rightarrow \Omega^{1}\left(M, T_{M}^{*}\right) .
$$

Note that (i) $\Omega^{0}\left(M, T_{M}^{*}\right) \cong \Omega^{1}(M)$ and (ii) $\Omega^{1}\left(M, T_{M}^{*}\right) \stackrel{\wedge}{\rightarrow} \Omega^{2}(M)$. The difference of $\wedge \circ \nabla: \Omega^{1}(M) \rightarrow \Omega^{2}(M)$ and the exterior derivative $d$ is given by a tensor, called the torsion tensor of $\nabla$. Explicitly $\operatorname{Tor}(\nabla) \in \Omega^{2}\left(M, T_{M}\right)$ is given by

$$
\operatorname{Tor}(\nabla)(X, Y)=\nabla_{X} Y-\nabla_{Y} X-[X, Y] .
$$

Geometrically, if $\nabla$ is any connection on $T_{M}^{*}$, then using parallel transports along radial paths from $x_{0} \in M$, we obtain a local trivialization $U \times$ $\mathbb{R}^{n} \stackrel{\simeq}{\rightarrow} T_{U}^{*} M$ given by $\left(x ; a_{1}, \ldots, a_{n}\right) \mapsto a_{1} s^{1}(x)+\cdots+a_{n} s^{n}(x)$ satisfying

$$
\nabla=d+O\left(|x|^{2}\right) \text {. }
$$

Here $x=\left(x^{1}, \ldots, x^{n}\right)$ are local coordinates on $M$ and $\left(s^{1}(x), \ldots, s^{n}(x)\right)$ are bases on fibers of $T^{*} M$. It is natural to require the compatibility that

$$
s^{1}(x)=d x^{1}, \ldots, s^{n}(x)=d x^{n} .
$$

This can be achieved precisely when $\operatorname{Tor}(\nabla)=0$.

In such a coordinate system, $g=\sum_{i, j} g_{i j}(x) d x^{i} \otimes d x^{j}$ has the following property

$$
g_{i j}=g\left(\frac{\partial}{\partial x^{i}}, \frac{\partial}{\partial x^{j}}\right)=\delta_{i j}+O\left(|x|^{2}\right),
$$

for small $|x|$. Namely $g_{i j}(0)=\delta_{i j}$ and $d g_{i j}(0)=0$. This is called a normal coordinate system and it comes handy when we perform tensor calculus on $M$. 
3.2. Classification of Riemannian holonomy groups. The holonomy group of the Levi-Civita connection of a Riemannian manifold is called the Riemannian holonomy group, or simply the holonomy group and we denote it as $\operatorname{Hol}(M, g)$. Up to conjugacy, it is a closed subgroup of $O(n)$. We will only consider the connected component of $\mathrm{Hol}(M, g)$. In this article we simply write $\mathrm{Hol}(M, g)$ for the identity component, sometimes called the reduced holonomy group. Every simply connected Riemannian manifold is a Riemannian product of those with irreducible holonomy groups and they are classified by Berger $[\mathbf{1 1}]$ as follows: Unless $(M, g)$ is a Riemannian symmetric space, $\operatorname{Hol}(M, g)$ must be either $G_{\mathbb{A}}(n)$ or $H_{\mathbb{A}}(n)$ for a normed division algebra $\mathbb{A}$, as given in the following table,

\begin{tabular}{|c||c|c|c|c|}
\hline $\mathbb{A}$ & $\mathbb{R}$ & $\mathbb{C}$ & $\mathbb{H}$ & $\mathbb{O}$ \\
\hline \hline$G_{\mathbb{A}}(n)$ & $O(n)$ & $U(n)$ & $S p(n) \operatorname{Sp}(1)$ & $\operatorname{Spin}(7)$ \\
\hline$H_{\mathbb{A}}(n)$ & $S O(n)$ & $S U(n)$ & $S p(n)$ & $G_{2}$ \\
\hline
\end{tabular}

The corresponding geometries are given as follows:

$\begin{array}{cccc}\text { Riemannian } & \text { Kähler } & \text { Quaternionic-Kähler } & \operatorname{Spin}(7) \\ \text { Volume } & \text { Calabi-Yau } & \text { Hyperkähler } & G_{2}\end{array}$

The geometry of manifolds with holonomy group $G_{\mathbb{A}}(n)$ or $H_{\mathbb{A}}(n)$ can be naturally interpreted as the geometry over the normed division algebra $\mathbb{A}$ and one with an $\mathbb{A}$-orientation respectively [93] (see section 10).

In section 9, we will also give another unified description of all these geometries in terms of real and complex vector cross products. Both approaches are closely related to each other and each has its own advantages.

Riemannian symmetric spaces are classified by Cartan in terms of Lie theory and the list is a bit longer. We will see in section 10 that they can be interpreted as Grassmannians of subspaces in a linear space defined over $\mathbb{A} \otimes \mathbb{B}$ for two normed division algebras $\mathbb{A}$ and $\mathbb{B}$.

3.3. Riemannian curvature tensors. The curvature tensor $R m$ of the Levi-Civita connection $\nabla$ of $(M, g)$ enjoys many nice properties. The condition $\nabla g=0$ implies that $R m \in \Omega^{2}\left(M, \Lambda^{2} T_{M}^{*}\right)$ and we can view it as a linear operator

$$
R m: \Lambda^{2} T_{M} \rightarrow \Lambda^{2} T_{M}
$$

called the curvature operator. This is a symmetric operator because of the torsion freeness of $\nabla$. Thus we can write $R m \in \Gamma\left(M, \operatorname{Sym}^{2}\left(\Lambda^{2} T_{M}^{*}\right)\right)$. 
Explicitly in term of local coordinate $x^{i}$ 's, if we write $g_{i j}=g\left(\frac{\partial}{\partial x^{\iota}}, \frac{\partial}{\partial x^{j}}\right)$, then the Levi-Civita connection $\nabla=d+\Gamma_{j k}^{i} d x^{j} \otimes d x^{k} \otimes \frac{\partial}{\partial x^{i}}$ is given by the formula

$$
\Gamma_{i j}^{k}=\frac{1}{2} g^{k l}\left(\frac{\partial g_{l j}}{\partial x^{i}}+\frac{\partial g_{i l}}{\partial x^{j}}-\frac{\partial g_{i j}}{\partial x^{l}}\right) .
$$

Here $\Gamma_{i j}^{k}$ 's are called the Christoffel symbols. The Riemannian curvature tensor is given by the formula

$$
-R m_{q k l}^{i}=\frac{\partial \Gamma_{q l}^{i}}{\partial x^{k}}-\frac{\partial \Gamma_{q k}^{i}}{\partial x^{l}}+\Gamma_{p k}^{i} \Gamma_{q l}^{p}-\Gamma_{p l}^{i} \Gamma_{q k}^{p},
$$

where $R m\left(\frac{\partial}{\partial x^{k}}, \frac{\partial}{\partial x^{l}}\right) \frac{\partial}{\partial x^{q}}=R m_{q k l}^{i} \frac{\partial}{\partial x^{i}}$ and we also write $R m_{p q k l}=g_{p i} R m_{q k l}^{i}$.

It has the following properties,

$$
\begin{aligned}
& 0=R m_{i j k l}+R m_{i j l k} \\
& 0=R m_{i j k l}-R m_{k l i j} \\
& 0=R m_{j k l}^{i}+R m_{k l j}^{i}+R m_{l j k}^{i} \\
& 0=R m_{i j k l, p}+R m_{i j l p, k}+R m_{i j p k, l} .
\end{aligned}
$$

If $R m$ is identically zero, then the universal cover of $M$ is the Euclidean space $\mathbb{R}^{n}$ with the standard flat metric. More generally, suppose $R m=\lambda I$ as an operator on $\Lambda^{2} T_{M}$, then the universal cover of $M$ must be the sphere or the hyperbolic space according to $\lambda$ being a positive or negative constant respectively. Such $M$ 's are called space forms. These are model spaces in geometry and they are examples of symmetric spaces,

$$
S^{n}=\frac{O(n+1)}{O(n)} \quad \text { and } \quad \mathbb{H}^{n}=\frac{O^{+}(n, 1)}{O(n)} .
$$

If we take the partial trace of $R m: \Lambda^{2} T_{M} \rightarrow \Lambda^{2} T_{M}$ by contracting one of the $T_{M}$-factor, then we obtain the Ricci tensor,

$$
R c: T_{M} \rightarrow T_{M}
$$

That is $R c_{i j}=R c_{j i}=R m_{i k j}^{k}$. The metric is called an Einstein metric if the Ricci curvature is constant. We can take the trace again to obtain the scalar curvature $R \in C^{\infty}(M)$, i.e. $R=g^{i j} R c_{i j}$.

There are a lot of studies on the interplay between curvature and topology of $M$. For instance, the Bochner formula

$$
\Delta \phi=\nabla^{*} \nabla \phi+R c(\phi)
$$

for one forms $\phi$ on $M$ implies that if $M$ has positive Ricci curvature, then there is no nontrivial harmonic one form. Using the Hodge theorem, this implies $H^{1}(M, \mathbb{R})=0$. 


\subsection{Flat tori.}

(Enhanced) moduli of flat tori. A flat torus $T^{n}$ is a quotient of the Euclidean space $\mathbb{R}^{n}$ by a lattice $\Gamma$. For instance $\mathbb{R}^{n} / \mathbb{Z}^{n}$ is the standard flat torus. The natural metric on $T^{n}$ has zero Riemannian curvature and every Einstein metric on $T^{n}$ is flat. The moduli space of flat metrics on $T^{n}$ is

$$
G L(n, \mathbb{Z}) \backslash G L(n, \mathbb{R}) / O(n) .
$$

This is because the space of lattices in $\mathbb{R}^{n}$ is $G L(n, \mathbb{Z}) \backslash G L(n, \mathbb{R})$ and changing a lattice by an orthogonal transformation will only change $T^{n}$ by an isometry. If we fix an isomorphism between $H^{1}\left(T^{n}, \mathbb{Z}\right)$ with $\mathbb{Z}^{n}$, called a marking, then the space of marked flat tori is $G L(n, \mathbb{R}) / O(n)$, which can also be identified as the space of positive definite inner products on $\mathbb{R}^{n}$.

Motivated from physics, we couple a flat metric with a $B$-field $B \in$ $H^{2}\left(T^{n}, U(1)\right)$, then the corresponding moduli space is the quotient of

$$
\frac{G L(n, \mathbb{R})}{O(n)} \times H^{2}\left(T^{n}, \mathbb{R}\right) \simeq \frac{S O^{+}(n, n)}{S O(n) \times S O(n)}
$$

by the discrete subgroup $G L(n, \mathbb{Z}) \times H^{2}\left(T^{n}, \mathbb{Z}\right)$. The above isomorphism is given by

$$
(\Lambda, B) \rightarrow\left(\begin{array}{cc}
\left(\Lambda^{t}\right)^{-1} & 0 \\
0 & \Lambda
\end{array}\right)\left(\begin{array}{cc}
I & -B \\
0 & I
\end{array}\right)
$$

We can further enhance these moduli spaces to the symmetric spaces for the split exceptional Lie groups of type $E_{n+1}$ provided $n+1 \leq 8$,

$$
\frac{E_{6}^{\text {split }}}{S p(4)}, \frac{E_{7}^{\text {split }}}{S U(8)} \text { and } \frac{E_{8}^{\text {split }}}{S O(16)} \text {. }
$$

Bundles over tori. The cohomology groups of $T^{n}=\mathbb{R}^{n} / \Gamma$ have natural isomorphisms,

$$
H^{k}\left(T^{n}, \mathbb{Z}\right) \simeq \bigwedge^{k} \Gamma^{*}
$$

where $\Gamma^{*}$ is the dual lattice to $\Gamma$. Moreover the Chern character map is an isomorphism over $\mathbb{Z}$, i.e.

$$
\operatorname{ch}: K\left(T^{n}\right) \stackrel{\simeq}{\rightarrow} H^{\text {even }}\left(T^{n}, \mathbb{Z}\right) .
$$

Every harmonic form on a flat torus is parallel. Indeed $d x^{i_{1}} \wedge d x^{i_{2}} \wedge \cdots \wedge$ $d x^{i_{k}}$ 's form a base of the space of harmonic forms on $T^{n}=\mathbb{R}^{n} / \Gamma$.

Flat $G$-bundles over $T^{n}$ are particularly easy to describe since $\pi_{1}\left(T^{n}\right) \cong$ $\mathbb{Z}^{n}$, at least when $G=U(r)$ or $n \leq 2$. Recall that the moduli space $\mathcal{M}^{G-\text { flat }}$ $\left(T^{n}\right)$ of flat $G$-bundles on any space can be identified with

$$
\mathcal{M}^{G \text {-flat }}\left(T^{n}\right) \cong \operatorname{Hom}\left(\pi_{1}\left(T^{n}\right), G\right) / A d(G) .
$$

We assume that $G$ is a compact Lie group. When $n=1$, i.e. $T^{n}$ is a circle, then $\mathcal{M}^{G \text {-flat }}\left(S^{1}\right)=G / A d(G)=T_{G} / W$, where $T_{G}$ is a maximal torus in $G$. This is because every element in $G$ can be conjugated to an element in $T_{G}$, unique up to the action of the Weyl group $W$. When $G=U(r)$, this says 
that every unitary matrix can be diagonalized uniquely up to permutations of eigenvalues. In this case, the Weyl group is the permutation group $S_{r}$ of $r$ elements.

Since $\pi_{1}\left(T^{n}\right)=\Gamma \cong \mathbb{Z}^{n}$ is a free Abelian group, $\operatorname{Hom}\left(\pi_{1}\left(T^{n}\right), G\right)$ is the set of commuting $n$-tuples in $G$. When $G=U(r)$, any such $n$-tuple of elements in $U(r)$ can be simultaneously diagonalized and therefore

$$
\mathcal{M}^{U(r) \text {-flat }}\left(T^{n}\right) \cong\left(\prod^{n} T_{G}\right) / S_{r} \simeq S y m^{r} T^{n *}
$$

is the $r^{\text {th }}$-symmetric power of $T^{n *}$.

However, for other $G,\left(\prod^{n} T_{G}\right) / W$ is only the largest connected component of $\mathcal{M}^{G-\text { flat }}\left(T^{n}\right)$. For $n=2$, a result of Borel says that

$$
\mathcal{M}^{G \text {-flat }}\left(T^{2}\right) \cong \frac{T_{G} \times T_{G}}{W}
$$

Suppose $T^{2}$ is given a complex structure, i.e. an elliptic curve $T_{\tau}^{2}$, Looijenga showed that these spaces are always complex weighted projective spaces. This result plays an important role in physics for the duality between heterotic string theory and F-theory.

When $G$ is the compact exceptional Lie group of type $E_{n}$, FriedmanMorgan-Witten and Donagi showed that $\mathcal{M}^{E_{n}-\text { flat }}\left(T_{\tau}^{2}\right)$ can be identified with the moduli space of degree $9-n$ del Pezzo surfaces with $T_{\tau}^{2}$ its anticanonical curve. This result was generalized by Leung-Zhang $[\mathbf{1 0 2}][\mathbf{1 0 3}]$ to compact Lie groups of any type.

$T$-duality. Let us come back to the case of flat $U(1)$-bundles over $T^{n}$. Their moduli space is canonically the dual torus $T^{n *} \simeq \mathbb{R}^{n *} / \Gamma^{*}$. Certainly $\left(T^{n *}\right)^{*}=T^{n}$. In fact, this duality between $T^{n}$ and its dual torus $T^{n *}$ can be applied to other structures on the tori, called the T-duality. The key observation lies in the existence of a universal $U(1)$-bundle $\mathcal{P}$ over the universal moduli space $T^{n} \times \mathcal{M}^{U(1)-\text { flat }}\left(T^{n}\right) \simeq T^{n} \times T^{n *}$, called the Poincaré bundle. It has a universal connection $\mathbf{D}$ in the sense that the restriction of $\mathbf{D}$ to any slice $T^{n} \times\left[D_{A}\right]$ is the flat $U(1)$-connection $D_{A}$ itself. On the level of differential forms, we define the Fourier-Mukai transformation $\mathcal{F}$ as follows,

$$
\begin{gathered}
\mathcal{F}: \Omega^{k}\left(T^{n}\right) \rightarrow \Omega^{n-k}\left(T^{n *}\right) \\
\mathcal{F}(\phi)=\int_{T^{n}} \phi \wedge e^{\frac{i}{2 \pi} \mathbf{F}}
\end{gathered}
$$

where $\mathbf{F}$ is the curvature two form of $\mathbf{D}$. We can rewrite this as

$$
\mathcal{F}(\phi)=\pi_{2 *}\left(\pi_{1}^{*} \phi \wedge e^{\frac{i}{2 \pi} \mathbf{F}}\right)
$$

where $\pi_{i}$ is the projection to the $i^{t h}$-factor in $T^{n} \times T^{n *}$. $\mathcal{F}$ descends to give an isomorphism between cohomology groups of $T^{n}$ and $T^{n *}$. Similar construction also give an isomorphism between their K-groups.

However, this is not an isomorphism on the level of differential forms. One can achieve this by incorporating the most basic duality transformation, 
namely the Fourier series, i.e. every periodic function $f(\theta)$ on $\mathbb{R}$ with period $2 \pi$ is a linear combination of $e^{i n \theta}$ 's,

$$
f(\theta)=\sum_{\nu \in \mathbb{Z}} \hat{f}(\nu) e^{i \nu \theta} .
$$

This can be viewed as a transformation from the function $f$ on $S^{1} \simeq \mathbb{R} / \mathbb{Z}$ to the function $\hat{f}$ on $\mathbb{Z}$. This transformation is an isometry between $L^{2}\left(S^{1}\right)$ and $l^{2}(\mathbb{Z})$. It can be easily generalized to give an isometry

$$
\mathcal{F}: L^{2}\left(T^{n}\right) \rightarrow l^{2}\left(\Gamma^{*}\right) .
$$

One can combine the Fourier-Mukai transformation with the Fourier series to give an isomorphism on the level of differential forms between $T^{n}$ and $T^{n *}[\mathbf{3 1}]$.

Notice that the Fourier-Mukai transformation of a flat $U(r)$-bundle over $T^{n}$ is $r$ points in $T^{n *}$ as $\mathcal{M}^{U(r) \text {-flat }}\left(T^{n}\right) \cong S y m^{r} T^{n *}$. In dimension four, there is another natural class of Yang-Mills connections, namely ASD connections (see section 4). In this case, the Fourier-Mukai transformation of an ASD connection (without flat factor) over $T^{4}$ is an ASD connection over $T^{4 *}[\mathbf{3 8}]$. By applying this transformation again, we get back the original connection.

3.5. Einstein metrics. The Einstein functional for any Riemannian metric $g$ on $M$ with volume one is given by its total scalar curvature,

$$
\int_{M} R d v_{g}
$$

In dimension two, this is a topological quantity, namely the Euler characteristic of the Riemann surface by the Gauss-Bonnet formula. In higher dimension, the Euler-Lagrange equation is the Einstein equation

$$
R c_{i j}=\frac{R}{2} g_{i j}
$$

and its solutions have constant Ricci curvature, i.e. Einstein metrics, which play very important roles in both geometry and general relativity.

In dimension two, Riemannian curvature tensor, Ricci curvature and scalar curvature are all the same. By rescaling a metric at each point, namely a conformal change, we can make its curvature constant. This is the uniformization theorem which identifies conformal structures (or equivalently complex structures) and Einstein metrics in this dimension.

In dimension three, every Einstein metric has constant sectional curvature. The search for them is an important avenue into understanding the Poincaré conjecture and Thurston geometrization conjecture for three dimensional topology and we have the recent breakthrough by Perelman toward solving them.

In dimension four, there are several topological obstructions to the existence of Einstein metrics. This situation is also similar to the Kähler case. In 
dimension five or higher, there are very few examples of Einstein manifolds. However, we do not have any topological constraint for them neither.

3.6. Minimal submanifolds. Given any submanifold $C$ in $M$, its tangent bundle is a subbundle of the restriction of $T_{M}$ to $C$ and the quotient bundle is called the normal bundle $N_{C / M}$, that is

$$
\left.0 \rightarrow T_{C} \rightarrow T_{M}\right|_{C} \rightarrow N_{C / M} \rightarrow 0
$$

Using a metric on $M$, we obtain an orthogonal decomposition

$$
\left.T_{M}\right|_{C}=T_{C} \oplus N_{C / M},
$$

and therefore an induced metric and an induced connection on $C$. This induced connection on $C$ is again torsion free, namely it is the Levi-Civita connection on $C$ with respect to the induced metric. We denote the second fundamental form as

$$
A \in \Omega^{1}\left(C, T_{C}^{*} \otimes N_{C / M}\right)=\Gamma\left(C, T_{C}^{*} \otimes T_{C}^{*} \otimes N_{C / M}\right) .
$$

The torsion freeness of $\nabla$ implies that $A=\sum A_{i j}^{\alpha} d x^{i} \otimes d x^{j} \otimes e_{\alpha}$ is a symmetric tensor,

$$
A \in \Gamma\left(C, \operatorname{Sym}^{2} T_{C}^{*} \otimes N_{C / M}\right),
$$

that is $A_{i j}^{\alpha}=A_{j i}^{\alpha}$ for any $i, j, \alpha$, where $e_{\alpha}$ 's is an orthogonal frame for the normal bundle. The mean curvature vector is the normal vector field obtained by taking trace of $A$ with respect to $g$, that is

$$
\vec{H}=\operatorname{Tr}_{g}(A)=\sum g^{i j} A_{i j}^{\alpha} e_{\alpha} \in \Gamma\left(C, N_{C / M}\right)
$$

The most natural class of oriented submanifolds $C$ in a Riemannian manifold $(M, g)$ consists of those with minimal volumes. Critical points for the volume functional are called minimal submanifolds and they are characterized by the vanishing of the mean curvature vector

$$
\vec{H}=0 \text {. }
$$

This second order quasi-linear system of elliptic differential equations has been extensively studied, especially when $C$ is either of codimension one or of dimension at most two.

When $C$ is an oriented hypersurface in $M$, there is a unique oriented normal vector field of unit length, denoted as $\hat{n}$. The mean curvature vector becomes a scalar function $H$ on $C$, that is $\vec{H}=H \hat{n}$, and the minimal hypersurface equation is a scalar equation which is much easier to handle than the general case. 
Calibrations. In the higher codimension cases, not much is known in general. However in the presence of additional geometric structures, for example Kähler, Calabi-Yau, $G_{2}$ or Spin (7) structure, that certain special classes of minimal submanifolds can be characterized by systems of first order equations. Moreover, their solutions are not just critical points for the volume functional, they are absolute volume minimizers. This is the theory of calibration, developed by Harvey and Lawson [59], based on the Wirtinger formula which gives the volume minimizing properties for complex submanifolds in Kähler manifolds.

A differential form $\phi \in \Omega^{k}(M)$ in a Riemannian manifold $M$ is called a calibration form if $d \phi=0$ and $\phi(P) \leq \operatorname{vol}(P)$ for any oriented $k$-plane $P$ in $T_{M}$. An important observation is that every $k$-dimensional submanifold $S$ in $M$ with $\left.\phi\right|_{S}$ equal its volume form for the induced metric is always a volume minimizing submanifold within its homology class $[S] \in H_{k}(M)$. This is because if $\left[S^{\prime}\right]=[S]$, we have $\int_{S} \phi=\int_{S^{\prime}} \phi$ using integrating by part and $d \phi=0$. Therefore

$$
\operatorname{Vol}(S)=\int_{S} \phi=\int_{S^{\prime}} \phi \leq \operatorname{Vol}\left(S^{\prime}\right) .
$$

Furthermore, any $S^{\prime}$ with the same volume as $S$ is also calibrated by $\phi$.

Examples include complex submanifolds in Kähler manifolds which are calibrated by $\omega^{k} / k$ !, special Lagrangian submanifolds in Calabi-Yau manifolds which are calibrated by $\operatorname{Re} \Omega$, associative (resp. coassociative) submanifolds in $G_{2}$-manifolds which are calibrated by $\Omega$ (resp. $* \Omega$ ) and Cayley submanifolds in $\operatorname{Spin}(7)$-manifolds which are calibrated by $\Theta$. The existence of these calibrations can be explained naturally in terms of vector cross products (see section 9).

3.7. Harmonic maps. Given any smooth map

$$
f: M \rightarrow N
$$

its linearization $d f(x): T_{x} M \rightarrow T_{f(x)} N$ at $x \in M$ determines a section

$$
d f \in \Gamma\left(M, T_{M}^{*} \otimes f^{*} T_{N}\right) .
$$

Given any Riemannian metrics $g_{M}$ and $g_{N}$ on $M$ and $N$ respectively, we can measure its length $|d f|(x) \geq 0$. The harmonic energy of $f$ is defined as follows,

$$
E(f)=\int_{M}|d f|^{2} d v_{g_{M}}
$$

The Euler-Lagrange equation is

$$
\Delta f=0 \in \Gamma\left(M, f^{*} T_{N}\right) .
$$


Here $\Delta f=\operatorname{Tr}_{g} \nabla(d f)$ with

$$
\nabla(d f) \in \Omega^{1}\left(M, T_{M}^{*} \otimes f^{*} T_{N}\right) \cong \Gamma\left(M, T_{M}^{*} \otimes T_{M}^{*} \otimes f^{*} T_{N}\right)
$$

the covariant derivative of the section $d f$.

When $N=\mathbb{R}$, harmonic maps are simply harmonic functions. When $N=$ $S^{1}$, any $f: M \rightarrow S^{1}$ defines a closed one form $f^{*}(d \theta)$ on $M$ representing a class in $H^{1}(M, \mathbb{Z})$. Thus harmonic maps to $S^{1}$ correspond to harmonic one forms representing classes in $H^{1}(M, \mathbb{Z})$.

Recall that (i) Hodge theorem says that every cohomology class has a unique harmonic form representative, which minimizes the energy and (ii) Bochner formula implies that harmonic one forms are zero if $R c_{M}>0$.

Harmonic maps behave like harmonic one forms. The Bochner formula can be generalized to

$$
\Delta|d f|^{2}=\frac{1}{2}|\nabla d f|^{2}+R c^{M}(d f, d f)-R m^{N}(d f, d f, d f, d f) .
$$

Using this formula, Eells-Sampson $[\mathbf{4 1}]$ showed that if $N$ is negatively curved, but not necessary compact, then any homotopy class of map $f: M \rightarrow N$ admits a unique harmonic map representative, which also minimizes the harmonic energy. If, moreover $R c^{M}>0$, then there is no nontrivial harmonic map at all. These Bochner type arguments for harmonic maps were refined by Siu, Yau and others to prove various superrigidity type theorems for locally symmetric spaces.

We note that holomorphic maps between Kähler manifolds are harmonic.

When $M$ is one dimensional, i.e. a circle $S^{1}$, then $f(M)$ is a geodesic in $N$ and the parametrization $f$ has constant speed. In general, harmonic maps and minimal submanifolds are quite different objects as the former one depends on the choice of $g_{M}$.

Sigma model. When $M$ is two dimensional, then the harmonic energy $E(f)$ is unchanged if we scale $g_{M}$ to $e^{u} g_{M}$ with $u$ any smooth function on $M$, i.e. $E(f)$ depends only on the conformal structure on $M$. The sigma model, or $\sigma$-model, in physics considers $E(f)$ as the action functional on the space of maps, or bosonic fields, $\operatorname{Map}(M, N)$ and this gives a conformal field theory. When we couple it with fermionic fields, i.e. spinors of $M$ twisted by $f^{*} T_{N}$, there is a supersymmetry (abbrev. $N=1$ SUSY) between bosons and fermions. When $N$ is a Kähler (resp. hyperkähler) manifold, then we can add more fermionic fields and obtain a $N=2$ (resp. $N=4$ ) SUSY $\sigma$-model.

In the Kähler case, we have

$$
E(f)=\int_{M}|d f|^{2}=\int_{M}|\partial f|^{2}+\int_{M}|\bar{\partial} f|^{2} .
$$

On the other hand,

$$
\int_{M} f^{*} \omega=\int_{M}|\partial f|^{2}-\int_{M}|\bar{\partial} f|^{2} .
$$


Thus

$$
E(f)=\int_{M} f^{*} \omega+2 \int_{M}|\bar{\partial} f|^{2}
$$

Note that $\int_{M} f^{*} \omega=[\omega](f(M))$ depends only on the homotopy class of the map $f$. Thus in a fixed homotopy class, holomorphic maps have the least harmonic energy $\int_{M} f^{*} \omega$. This continues to hold true for any symplectic manifold $M$ with a compatible metric. Similar situations also happen for gauge theory over an oriented Riemannian four manifold (section 4.1).

Witten [134] studied twisted versions of $N=2 \sigma$-models and defined two different models of TFT, called the $A$-model and $B$-model. The A-model describe the quantum symplectic geometry of $N$ and Gromov-Witten invariants (section 5.4) are partition functions in this model. The B-model describe the complex geometry of $N$ and there are no instanton effects.

When $N$ is a Calabi-Yau manifold, the mirror symmetry conjecture says that there should be another Calabi-Yau manifold $N^{\prime}$ such that the A-model and the B-model on $N$ and $N^{\prime}$ got interchanged (section 6.3).

\section{Oriented four manifolds}

Oriented manifolds of dimension two are special as they are one dimensional complex manifolds, i.e. Riemann surfaces. This is because $S O(2) \cong$ $U$ (1). Similarly, oriented manifolds of dimension four are one dimensional quaternionic manifolds because $S O(4)=S p(1) S p(1)$. As $\Lambda^{2} \mathbb{R}^{4} \cong \mathbf{s p}(1) \oplus$ $\mathbf{s p}(1) \cong \mathbf{s o}(4)$, given any oriented Riemannian four manifold $M$, we have a decomposition of its two forms into self-dual (SD) and anti-self-dual (ASD) components,

$$
\Lambda^{2}(M)=\Lambda_{+}^{2}(M) \oplus \Lambda_{-}^{2}(M) .
$$

Indeed this corresponds to the eigenspace decomposition for the Hodge star operator $*$ on the space of two forms. There is a corresponding decomposition for harmonic two forms, via Hodge theory we have

$$
H^{2}(M)=H_{+}^{2}(M) \oplus H_{-}^{2}(M)
$$

This decomposition depends only on the conformal structure of $M$. Note that

$$
\int_{M} \phi \wedge \phi= \pm \int_{M}|\phi|^{2}
$$

for $\phi \in H_{ \pm}^{2}(M)$. Thus the intersection product $q_{M}$ on $H^{2}(M)$ is positive (resp. negative) definite on $H_{+}^{2}(M)$ (resp. $\left.H_{-}^{2}(M)\right)$ The signature $\tau(M)=$ $\operatorname{dim} H_{+}^{2}(M)-\operatorname{dim} H_{-}^{2}(M)$ is a topological invariant of $M$ and it equals to the characteristic number $p_{1}(M) / 3$ by the Hirzebruch signature formula. 
4.1. Gauge theory in dimension four.

Donaldson theory. First we note that the Yang-Mills energy functional

$$
\int_{M}\left|F_{A}\right|^{2}
$$

on an oriented Riemannian four manifold $(M, g)$ depends only on the conformal class of the metric $g$.

As curvature tensors are matrix-valued two forms, for Hermitian connections $D_{A}$ on any complex vector bundle $E$ over an oriented Riemannian four manifold $M$, we have

$$
F_{A}=F_{A}^{+}+F_{A}^{-}
$$

with

$$
F_{A}^{ \pm}:=\frac{1}{2}\left(F_{A} \pm * F_{A}\right) \in \Omega_{ \pm}^{2}(M, a d(E)),
$$

the (anti-)self-dual (abbrev. SD/ASD) components of $F_{A}$. Notice that

$$
\int_{M}\left|F_{A}\right|^{2}=\int_{M}\left|F_{A}^{+}\right|^{2}+\int_{M}\left|F_{A}^{-}\right|^{2}
$$

and when $c_{1}(E)=0$, we also have

$$
\begin{aligned}
c_{2}(E) & =\frac{-1}{8 \pi^{2}} \int_{M} \operatorname{Tr} F_{A} \wedge F_{A} \\
& =\frac{-1}{8 \pi^{2}} \int_{M}\left|F_{A}^{+}\right|^{2}+\frac{1}{8 \pi^{2}} \int_{M}\left|F_{A}^{-}\right|^{2} .
\end{aligned}
$$

Thus the Yang-Mills energy functional satisfies,

$$
\int_{M}\left|F_{A}\right|^{2}=8 \pi^{2} c_{2}(E)+2 \int_{M}\left|F_{A}^{+}\right|^{2} .
$$

Therefore, when $c_{2}(E) \geq 0$, the absolute minimal for the Yang-Mills energy is realized by ASD connections, i.e. $F_{A}^{+}=0$, also called instantons.

For a line bundle over $\mathbb{R}^{3,1}$, the ASD-equation

$$
F_{A}^{+}=0
$$

is the Maxwell equation for electromagnetism. In general, when $E$ is a line bundle, this linear equation is well-understood via Hodge theory. The situation is much more complicated when the rank $r$ of $E$ is higher as the moduli space of solutions is not compact. When $r$ equals two, using foundational works of Uhlenbeck, Taubes and others, Donaldson [38] studied the intersection theory on this moduli space and defined new and powerful invariants for the differentiable structures on four manifolds. When $M$ is a Kähler surface, then the instanton equation is the same as the Hermitian Yang-Mills equation with zero slope. Donaldson [36] showed that this latter equation is solvable precisely when $E$ is a polystable holomorphic vector bundle over $M$. In higher dimensions, this result is proved by Uhlenbeck-Yau [125]. 
Witten $[\mathbf{1 3 2}]$ described the Donaldson invariants as partition functions of a topological field theory (TFT) with $N=2$ supersymmetries. This TFT links the 4-dimensional Donaldson theory and the 3-dimensional ChernSimons Floer theory and 2-dimensional gauge theory as explained by Atiyah $[\mathbf{3}]$.

Seiberg-Witten theory. After much work from both mathematicians and physicists, Witten [132] found that the Donaldson theory should be equivalent to a much simpler theory, the so-called Seiberg-Witten (SW) theory. In this theory, $D_{A}$ is a connection of a line bundle $L$. The $\mathrm{SW}$-equation is

$$
\begin{aligned}
F_{A}^{+} & =\sigma(\phi) \\
\mathcal{D}_{A} \phi & =0 .
\end{aligned}
$$

Here $\phi \in S_{L}^{+}$is a positive spinor field twisted by $L$. The operator $\mathcal{D}_{A}$ is the

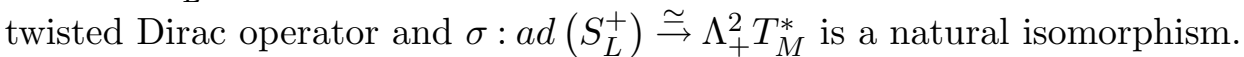

The Weitzenbock formula

$$
\mathcal{D}_{A}^{*} \mathcal{D}_{A} \phi=D_{A}^{*} D_{A} \phi+\frac{R}{4} \phi+F_{A}^{+} \cdot \phi
$$

implies that the SW-equation has no nontrivial solution if $M$ has positive scalar curvature. It can also be used to show that the moduli space of solutions to the SW-equation is compact. This makes the SW theory much simpler than the Donaldson theory. The compactness of the moduli space of SW equation still holds true even if we allow the spinor field $\phi$ to have eigencomponents by Leung-Xu [100] using an eigenvalue estimate by VafaWitten $[\mathbf{1 2 6}]$.

SW theory has many important applications in different branches of four dimensional theory, including (i) differential topology, for instance the Thom conjecture [78], (ii) Kähler geometry, (iii) Riemannian geometry, for instance the study of Einstein metric $[\mathbf{8 0}][\mathbf{8 7}][\mathbf{9 0}]$ and (iv) symplectic geometry, for instance $\mathrm{SW}=\mathrm{GW}$ by Taubes and classification of symplectic four manifolds with $b_{+}=1[\mathbf{1 0 4}][\mathbf{1 1 8}]$.

Vafa-Witten theory. Vafa and Witten also studied a $N=4$ TFT on four manifolds in $[\mathbf{1 2 7}]$ and derived the Vafa-Witten equation

$$
\begin{aligned}
F_{A}^{+}+[B, B]+[C, B] & =0 \\
D_{A}^{*} B+D_{A} C & =0,
\end{aligned}
$$

where $B \in \Omega_{+}^{2}(M, a d(E))$ and $C \in \Omega^{0}(M, a d(E))$. The S-duality in physics predicts that the generating function for the Euler characteristics of these moduli spaces is a modular form. More recently, Vafa-Witten introduced another $N=4$ TFT [127] and conjectured that S-duality for this theory would give a physical explanation of the Langlands program.

4.2. Riemannian geometry in dimension four. Recall that the Riemannian curvature tensor $R m$ is a self-adjoint operator on $\Lambda^{2} T_{M}^{*}$. With 
respect to the decomposition of two forms into SD and ASD components, we have

$$
R m=\left(\begin{array}{cc}
W^{+}+R / 12 & R c^{0} \\
R c^{0} & W^{-}+R / 12
\end{array}\right) .
$$

Here $R$ is the scalar curvature, $R c^{0}$ is the tracefree part of the Ricci curvature and $W=W^{+}+W^{-}$is the Weyl curvature (section 12) and it depends only on the conformal structure on $M$. When $W=0, M$ is conformally flat. $M$ is called an ASD (resp. SD) manifold if $W^{+}=0$ (resp. $W^{-}=0$ ). Note that if we reverse the orientation of $M$, then $W^{+}$and $W^{-}$get interchanged. The signature $\tau(M)=\operatorname{dim} H_{+}^{2}(M)-\operatorname{dim} H_{-}^{2}(M)$ can be expressed as

$$
\tau(M)=\frac{1}{12 \pi^{2}} \int_{M}\left|W^{+}\right|^{2}-\left|W^{-}\right|^{2} .
$$

When $M$ is an Einstein manifold, the Euler characteristic $\chi(M)$ can be computed as follows,

$$
\chi(M)=\frac{1}{8 \pi^{2}} \int_{M}\left|W^{+}\right|^{2}+\left|W^{-}\right|^{2}+R^{2} / 24 .
$$

In particular, we obtain the Hitchin inequality

$$
\tau(M) \leq \frac{2}{3} \chi(M)
$$

where the equality sign holds if and only if $W^{-}=R=0$. When $M$ is Kähler, this inequality can be rewritten as $c_{1}^{2}(M) \leq 4 c_{2}(M)$. In the Kahler-Einstein case, it can be sharpened to

$$
c_{1}^{2}(M) \leq 3 c_{2}(M),
$$

the Miyaoka-Yau inequality. The equality sign holds if and only if the universal cover of $M$ is the complex ball $B_{\mathbb{C}}^{2}=S U(2,1) / U(2)$. Note that Yau showed that every Kähler manifold with $c_{1}(M)=0$ or $<0$ admits a KählerEinstein metric. Thus this inequality is an important tool in the study of the classification problem of complex algebraic surfaces. Lebrun [80] generalized the Miyaoka-Yau inequality to the real case with the help of the Seiberg-Witten invariants. If the Seiberg-Witten invariant is nonzero with respect to the reversed orientation on a Kähler-Einstein surface, then the index $\tau(M)$ is nonnegative, and it is zero if and only if the universal cover of $M$ is covered by the product of complex disks $B_{\mathbb{C}}^{1} \times B_{C}^{1}=(S U(1,1) / U(1))^{2}$. Recall that $B_{\mathbb{C}}^{2}$ and $B_{\mathbb{C}}^{1} \times B_{C}^{1}$ are the only Hermitian symmetric spaces of noncompact type in this dimension [87]. There is also a non-Hermitian symmetric space, namely the hyperbolic ball $B^{4}$, for which one can also find a characterization using Chern number inequality by using the non-Abelian Seiberg-Witten equation [90]. 
Twistor transform. We can associate to every oriented Riemannian four manifold $M$ a 6-dimensional manifold $Z$, called the twistor space of $M$, which is equipped with an almost complex structure $J_{Z}$. It is the total space of a fiber bundle

$$
S^{2} \rightarrow Z \stackrel{\pi}{\rightarrow} M
$$

with fiber over $x \in M$ being the set of all linear orthogonal complex structures on $T_{x} M$, which is a copy of $S O(4) / U(2) \simeq S^{2}$. For instance, when $M=S^{4}$, we have $Z=\mathbb{C P}^{3}$ and the fibration is given by sending a complex line in $\mathbb{C}^{4}$ to the quaternionic line in $\mathbb{H}^{2}$ that it spans as $S^{4}=\mathbb{H} \mathbb{P}^{1}$.

The necessary and sufficient condition for $\left(Z, J_{Z}\right)$ to be a complex manifold is $W^{+}=0$ on $M$.

Every Kähler surface with zero scalar curvature is an ASD 4-manifold. Taubes $[\mathbf{1 1 7}]$ showed that after taking connected sum with sufficiently many copies of $\overline{\mathbb{C P}^{2}}$, every $M$ admits an ASD metric.

Atiyah, Hitchin and Singer [4] showed that if $M$ is an ASD 4-manifold, then the conformal geometry of $M$ can be described in terms of the complex geometry of $Z$, called the twistor transformation. We will discuss a particular interesting class of ASD four manifolds, namely the K3 surfaces, in section 6.4.

\section{Kähler geometry}

A Kähler manifold is a Riemannian manifold $M$ with a compatible complex structure. Recall that $M$ is a complex manifold if it has a covering by complex coordinate charts with holomorphic transition functions. In the linear setting, a complex vector space is equivalent to a real vector space $V$ with a $\mathbb{R}$-linear homomorphism $J: V \rightarrow V$ satisfying $J^{2}=-1$. It is compatible with an inner product $g$ on $V$ if $g$ is Hermitian, i.e. for any $u, v \in V$ we have $g(J u, J v)=g(u, v)$. In this case, we have a non-degenerate two form $\omega \in \Lambda^{2} V^{*}$ defined by

$$
\omega(u, v)=g(J u, v) .
$$

Thus $V$ is a symplectic vector space. Indeed any two of these structures $g$, $J$ and $\omega$ determines the third one, i.e. the intersection of any two of the three subgroups $O(2 n), G L(n, \mathbb{C})$ and $S p(2 n, \mathbb{R})$ in $G L(2 n, \mathbb{R})$ is always the unitary group $U(n)$.

On a manifold $M$, an almost complex structure is a linear complex structure $J_{x}$ on every tangent space $T_{x} M . M$ is called a Kähler manifold if $g$ is Hermitian and $J$ is parallel, $\nabla J=0$. Equivalently a Kähler manifold is a Riemannian manifold with holonomy $U(n)$.

A Kähler manifold is always a complex manifold by the theorem of Newlander and Nirenberg which states that an almost complex structure is integrable, i.e. coming from the linearization of a complex structure on $M$ if and only if the Nijenhuis tensor $N \in \Omega^{2}(M, T M)$ vanishes, where

$$
4 N(u, v)=[u, v]+J[J u, v]+J[u, J v]-[J u, J v]
$$


for any $u, v \in T_{x} M$ and for any $x \in M$. In particular, if $J$ is parallel with respect to some torsion free connection $\nabla$, say the Levi-Civita connection, then $M$ is a complex manifold.

An important observation is that if $M$ is already a Hermitian complex manifold, then the Kählerian condition $\nabla J=0$, or equivalently $\nabla \omega=0 \in$ $\Omega^{2}\left(M, T_{M}\right)$, can be reduced to the closedness of the Kähler form, $d \omega=0 \in$ $\Omega^{3}(M)$. This implies that every complex submanifold of a Kähler manifold is always Kähler. In particular, every projective algebraic manifold $M$ in $\mathbb{C P}^{N}$ is Kähler, thus providing abundant examples of Kähler manifolds. Kodaira embedding theorem gives a necessary and sufficient condition for a compact Kähler manifold $M$ to be projective, namely the Kähler class $[\omega] \in H^{2}(M, \mathbb{R})$ should be defined over $\mathbb{Z}$. As a result, Kähler geometry and complex algebraic geometry are intimately related to each other.

\subsection{Kähler geometry - complex aspects.}

Dolbeault cohomology and Hodge $(p, q)$-decomposition. A linear complex structure on a real vector space $V$ can be rephrased as a decomposition $V \otimes \mathbb{C}=V^{1,0} \oplus V^{0,1}$ satisfying $\overline{V^{0,1}}=V^{1,0}$. Indeed $V^{1,0}$ and $V^{0,1}$ are the $\pm i$ eigenspaces of $J$. Taking tensor powers, we have

$$
\bigwedge^{k} V \otimes \mathbb{C}=\bigoplus_{p+q=k} V^{p, q} \quad \text { with } \overline{V^{q, p}}=V^{p, q} .
$$

Here $V^{p, q}=\bigwedge^{p} V^{1,0} \otimes \bigwedge^{q} V^{0,1}$. We have a corresponding decompositions for differential forms on any almost complex manifold $M$,

$$
\Omega^{k}(M, \mathbb{C})=\bigoplus_{p+q=k} \Omega^{p, q}(M) \quad \text { with } \overline{\Omega^{q, p}(M)}=\Omega^{p, q}(M) .
$$

The exterior differentiation $d: \Omega^{k}(M, \mathbb{C}) \rightarrow \Omega^{k+1}(M, \mathbb{C})$ decomposes into $d=\partial+N+\bar{\partial}+\bar{N}$ with $\partial: \Omega^{p, q}(M) \rightarrow \Omega^{p+1, q}(M)$ and $N: \Omega^{p, q}(M) \rightarrow$ $\Omega^{p+2, q-1}(M)$ being the tensor product with the Nijenhuis tensor. $M$ being a complex manifold, i.e. $N=0$, is equivalent to

$$
\bar{\partial}^{2}=0: \Omega^{p, q}(M) \rightarrow \Omega^{p, q+2}(M) .
$$

That is, we have an elliptic complex

$$
0 \rightarrow \Omega^{0,0}(M) \stackrel{\bar{\partial}}{\rightarrow} \Omega^{0,1}(M) \stackrel{\bar{\partial}}{\rightarrow} \cdots \stackrel{\bar{\partial}}{\rightarrow} \Omega^{0, n}(M) \rightarrow 0 \quad \text { with } \bar{\partial}^{2}=0,
$$

called the Dolbeault complex.

The corresponding cohomology group is called the Dolbeault cohomology, denoted

$$
H_{\bar{\partial}}^{p, q}(M)=\left.\frac{\operatorname{Ker}(\bar{\partial})}{\operatorname{Im}(\bar{\partial})}\right|_{\Omega^{p, q}(M)} .
$$

Its dimensions $h^{p, q}(M)=\operatorname{dim} H_{\bar{\partial}}^{p, q}(M)$ 's are called the $(p, q)$-Hodge numbers of $M$. 
Similarly, on any holomorphic vector bundle $E$ over $M$, there is a twisted $\bar{\partial}$ operator,

$$
\bar{\partial}_{E}: \Omega^{p, q}(M, E) \rightarrow \Omega^{p, q+1}(M, E) \quad \text { with } \bar{\partial}_{E}^{2}=0,
$$

and the corresponding cohomology groups are denoted as $H_{\bar{\partial}}^{p, q}(M, E)$. There is a canonical identification $H_{\bar{\partial}}^{p, q}(M, E) \cong H_{\bar{\partial}}^{0, q}\left(M, \Lambda^{p} T_{M}^{*} \otimes E\right)$ and we simply write it as $H^{q}\left(M, \Lambda^{p} T_{M}^{*} \otimes E\right)$. Analogous to the Poincaré duality, the Serre duality gives the isomorphism

$$
H^{p, q}(M, E) \cong H^{n-p, n-q}\left(M, E^{*}\right)^{*} .
$$

In particular,

$$
H^{q}(M, E) \cong H^{n-q}\left(M, K_{M} \otimes E^{*}\right)^{*} .
$$

If we endow both $M$ and $E$ with Hermitian metrics, then we can define the twisted Laplacian operator

$$
\Delta_{\bar{\partial}_{E}}=\bar{\partial}_{E} \bar{\partial}_{E}^{*}+\bar{\partial}_{E}^{*} \bar{\partial}_{E},
$$

where $\bar{\partial}_{E}^{*}$ is the adjoint of $\bar{\partial}_{E}$ and the Hodge theorem of representing cohomology classes by harmonic forms has a direct generalization here which gives an isomorphism

$$
H_{\bar{\partial}}^{p, q}(M, E) \cong \operatorname{Ker}\left(\left.\Delta_{\bar{\partial}_{E}}\right|_{\Omega^{p, q}(M, E)}\right) .
$$

When $M$ is a Kähler manifold, one can prove that $\Delta=2 \Delta_{\bar{\partial}}$ and this has many important consequences. For instance, we have the following Hodge $(p, q)$-decomposition,

$$
H^{k}(M, \mathbb{C})=\bigoplus_{p+q=k} H_{\bar{\partial}}^{p, q}(M) \text { with } \overline{H_{\bar{\partial}}^{q, p}(M)}=H_{\bar{\partial}}^{p, q}(M) .
$$

In particular, every odd degree Betti number of a compact Kähler manifold is even, $b_{2 l+1}(M) \in 2 \mathbb{Z}$.

This collection of subspaces $H^{p, q}(M)$ 's in $H^{k}(M, \mathbb{Z}) \otimes \mathbb{C}$ is called the Hodge structure of $M$. It depends only on the complex structure on $M$, but not on the choice of Kähler metrics and it carries important informations about the complex structure.

Hard Lefschetz action. Recall that $\omega(u, v)=g(J u, v)$ defines a symplectic structure on $M$. In particular, $[\omega]^{l} \neq 0 \in H^{2 l}(M, \mathbb{R})$ for any $0 \leq l \leq n$. Let

$$
\begin{gathered}
L: \Omega^{k}(M) \rightarrow \Omega^{k+2}(M) \\
L(\phi)=\phi \wedge \omega
\end{gathered}
$$

and $\Lambda$ be its adjoint operator. Then $H=[L, \Lambda]: \Omega^{k}(M) \rightarrow \Omega^{k}(M)$ is a multiple of the identity operator, $H=(n-k)$. The relationships

$$
[H, L]=2 L, \quad[H, \Lambda]=-2 \Lambda \quad \text { and } \quad[L, \Lambda]=H
$$

defines an $\operatorname{sl}(2, \mathbb{R})$-action on $\Omega^{*}(M)$. The closedness of $\omega$ implies that $L$ can be descended to $H^{*}(M, \mathbb{R})$ and the Kähler property of $M$, i.e. $\omega$ is 
parallel, implies that both $L$ and $\Lambda$ commute with $\Delta$ and therefore the above sl $(2, \mathbb{R})$-action can be descended to $H^{*}(M, \mathbb{R})$, called the Hard Lefschetz action. This action allows us to recover $H^{*}(M, \mathbb{R})$ from $\operatorname{Ker}(\Lambda)$, called the primitive cohomology and to reduce the Hodge structure on $H^{*}(M, \mathbb{C})$ to the primitive cohomology and define a polarized Hodge structure.

Kähler identities. First order Kähler identities give relationships between zeroth order operators $L, \Lambda, H$ and first order operators $\partial, \bar{\partial}, \partial^{*}$, $\bar{\partial}^{*}$. They are

$$
\begin{aligned}
{\left[L, \partial^{*}\right] } & =i \bar{\partial}, \quad\left[L, \bar{\partial}^{*}\right]=-i \partial \\
{[\Lambda, \partial] } & =i \bar{\partial}^{*}, \quad[\Lambda, \bar{\partial}]=-i \partial^{*}
\end{aligned}
$$

and all other brackets are zero. Indeed these formulas hold true even when $J$ is only an almost complex structure, namely $M$ is a symplectic manifold with a compatible almost complex structure $J$, sometimes called an almost Kähler structure.

Second order Kähler identities are

$$
\Delta=2 \Delta_{\bar{\partial}}=2 \Delta_{\partial},
$$

and $\Delta$ commutes with $L, \Lambda, H, \partial, \bar{\partial}, \partial^{*}$ and $\bar{\partial}^{*}$. We have seen earlier that the identity $\Delta=2 \Delta_{\bar{\partial}}$ is used to define the Hodge structure on $H^{*}(M, \mathbb{C})$.

Recall that the identities among zeroth order operators $L, \Lambda, H$ define a $\operatorname{sl}(2, \mathbb{R})$-action on $\Omega^{*}(M)$ and there is a canonical identification between Lie algebras $s l(2, \mathbb{R}) \cong s u(1,1)$. Indeed all the above identities together define an action on $\Omega^{*}(M, \mathbb{C})$ by the super Lie algebra

$$
s u(1,1)_{\text {sup }}=s u(1,1) \oplus \mathbb{C}^{1,1} \oplus \mathbb{R}
$$

with $\mathbb{C}^{1,1}$ spanned by $\partial, \bar{\partial}, \partial^{*}, \bar{\partial}^{*}$ and $\mathbb{R}$ spanned by $\Delta$.

Indeed this super Lie algebra action encompasses the hard Lefschetz action and all the Kähler identities. It has natural generalizations to manifolds defined over other normed division algebras (section 10).

Deformation of complex structures. A complex structure on $M$ can be characterized by the $\bar{\partial}$-operator

$$
\bar{\partial}: \Omega^{p, q}(M) \rightarrow \Omega^{p, q+1}(M)
$$

satisfying the integrability condition $(\bar{\partial})^{2}=0$. A different complex structure is then given by

$$
\bar{\partial}+\phi \quad \text { with } \phi \in \Omega^{0,1}\left(M, T_{M}\right) .
$$

The integrability condition becomes

$$
\bar{\partial} \phi+[\phi, \phi]=0 .
$$

For a smooth family of complex structures $\bar{\partial}+\phi(t)$,

$$
\phi(t)=t \phi_{1}+t^{2} \phi_{2}+t^{3} \phi_{3}+\cdots
$$


we have

$$
\begin{aligned}
& \bar{\partial} \phi_{1}=0 \\
& \bar{\partial} \phi_{2}=-\left[\phi_{1}, \phi_{1}\right] \text { and so on. }
\end{aligned}
$$

The first equation says that infinitesimal deformations of complex structures are parametrized by $\Omega^{0,1}\left(M, T_{M}\right) \cap \operatorname{Ker}(\bar{\partial})$. Up to diffeomorphisms, they are parametrized by the cohomology group $H^{1}\left(M, T_{M}\right)$. When $H^{2}$ $\left(M, T_{M}\right)=0$, the second equation for $\phi_{2}$ can always be solved and the same is true for all other $\phi_{j}$ 's. Using the Hodge theory for any given $\phi_{1}$, we can obtain a convergent power series solution $\phi(t)$ for small $t$ and therefore we have an honest family of deformations of the complex structure $\bar{\partial}$. This means that the moduli space of complex structures is smooth and with tangent space $H^{1}\left(M, T_{M}\right)$ at $\bar{\partial}$.

Even when $H^{2}\left(M, T_{M}\right) \neq 0$, the integrability condition can sometimes be solved for any given $\phi_{1}$. For example, this is the case for Calabi-Yau manifolds. In general there is a Kurinishi (nonlinear) map $\kappa$ defined on a neighborhood of the origin,

$$
\kappa: H^{1}\left(M, T_{M}\right) \rightarrow H^{2}\left(M, T_{M}\right),
$$

such that the moduli space of complex structures on $M$ near $\bar{\partial}$ is given by $\kappa^{-1}(0)$. The map satisfies $\kappa(\phi)=[\phi, \phi]+O\left(|\phi|^{3}\right)$. The space $H^{2}\left(M, T_{M}\right)$ is called the obstruction space for the deformations.

For a holomorphic bundle $E$ over a fixed complex manifold $M$, infinitesimal deformations of $E$ are parametrized by $H^{1}(M, E n d(E))$ and the obstruction space is given by $H^{2}(M, E n d(E))$. There are also analogous spaces for deformations of flat bundles.

For a holomorphic map $f: C \rightarrow M$, the space of infinitesimal deformations (resp. obstruction space) of $f$ with fixed $M$ is given by $H^{0}\left(C, N_{f(C) / M}\right)$ (resp. $H^{1}\left(C, N_{f(C) / M}\right)$ )

We remark that the deformation theory only deal with the local structure of moduli spaces. Construction of a global moduli space as a complex variety, or projective variety, is a different matter, which is often dealt with via the geometric invariant theory.

Subvarieties and coherent sheaves. We remark that on any Kähler manifold $M$, the form $\omega^{k} / k ! \in \Omega^{2 k}(M)$ is always a calibration form and those submanifolds calibrated by it are precisely complex submanifolds in $M$. This follows from the Wirtinger formula. As a corollary, complex submanifolds $S$ in Kähler manifolds are volume minimizers and they define nontrivial cohomology classes because $\int_{S} \omega^{k} / k$ ! is the volume of $S$,

$$
P D[S] \in H_{\bar{\partial}}^{p, p}(M) \cap H^{2 p}(M, \mathbb{Z}),
$$

where $p=n-k$ is the complex codimension of $S$. This continues to hold true even when $S$ is singular, namely a subvariety in $M$. The famous Hodge 
conjecture asks that whether every class in $H_{\bar{\partial}}^{p, p}(M) \cap H^{2 p}(M, \mathbb{R})$ is represented a $\mathbb{Q}$-linear combination of subvarieties in a projective manifold $M$.

The Chern classes $c_{p}(E)$ for any holomorphic vector bundle also lie in $H_{\bar{\partial}}^{p, p}(M) \cap H^{2 p}(M, \mathbb{Z})$ and this continues to hold true for any coherent sheaf, which allows $E$ to be singular. Quasi-isomorphism classes of complexes of coherent sheaves form a derived category $D^{b}(M)$. It contains much information about $M$, for instance, it determines $M$ completely when $c_{1}(M)$ is either positive or negative [15].

\subsection{Kähler geometry—Riemannian aspects.}

Hermitian Yang-Mills metrics. Given any Hermitian metric $h_{E}$ on a holomorphic vector bundle $E$ over a complex manifold $M$, there is a unique Hermitian connection $D_{E}$ satisfying $D_{E} h_{E}=0$ and

$$
\left(D_{E}\right)^{0,1}=\bar{\partial}_{E}: \Omega^{0}(M, E) \rightarrow \Omega^{0,1}(M, E) .
$$

In terms of any local holomorphic frame $e_{i}$ 's on $E$, we have

$$
\begin{aligned}
D_{E} & =d+h^{-1} \partial h, \text { and } \\
F_{E} & =\bar{\partial}\left(h^{-1} \partial h\right) \in \Omega^{1,1}(M, a d(E)),
\end{aligned}
$$

where $h=\left(h_{i \bar{j}}\right)$ with $h_{i \bar{j}}=h_{E}\left(e_{i}, e_{j}\right)$. This implies that

$$
c_{1}(E)=\frac{i}{2 \pi}\left[\operatorname{Tr}_{E}\left(F_{E}\right)\right]=\frac{i}{2 \pi}[\bar{\partial} \partial \log \operatorname{det} h]
$$

and Chern classes are of type $(p, p)$, i.e. $c_{p}(E) \in H^{p, p}(M) \cap H^{2 p}(M, \mathbb{Z})$.

For holomorphic bundles over a Kähler manifold, the Yang-Mills equation $D_{E}^{*} F_{E}=0$ is equivalent to $D_{E}\left(\Lambda F_{E}\right)=0$ because of the Kähler identity $[\Lambda, \bar{\partial}]=-i \partial^{*}$. Thus an eigenbundle in $E$ for the bundle endomorphism $\Lambda F_{E}$ is a holomorphic (parallel) subbundle. Thus, unless $E$ is reducible, the Yang-Mills equation reduces to a first order differential equation, called the Hermitian-Yang-Mills equation,

$$
\Lambda F_{E}=\mu_{E} I
$$

where the constant $\mu_{E}$ is called the slope of $E$ and it is given by ${ }^{2}$

$$
\mu_{E}=\frac{1}{r} \int_{M} c_{1}(E) \wedge \frac{\omega^{n-1}}{(n-1) !},
$$

where $r$ is the rank of $E$.

We remark that when $M$ is a Kähler surface, then the equations $F_{E}^{0,2}=$ $\Lambda F_{E}=0$ is the same as the ASD equation for unitary connections on the four manifold $M$. This is because $\Lambda_{+}^{2} \cong \mathbb{R} \omega \oplus \operatorname{Re} \Lambda^{0,2} \oplus \operatorname{Im} \Lambda^{0,2}$.

\footnotetext{
${ }^{2}$ For simplicity, we have normalized the volume of $M$ to be one.
} 
A Hermitian-Yang-Mills connection is not just a critical point for the Yang-Mills functional $\int\left|F_{E}\right|^{2}$, it is an absolute minimizer! This can be seen from the following equality, derived from the Chern-Weil theory,

$$
\begin{aligned}
\int_{M}\left|F_{E}-\frac{1}{r} \operatorname{Tr}\left(F_{E}\right) I\right|^{2} \frac{\omega^{n}}{n !}= & \int_{M}\left|\Lambda F_{E}-\frac{1}{r} \operatorname{Tr}\left(\Lambda F_{E}\right) I\right|^{2} \frac{\omega^{n}}{n !} \\
& -\frac{4 \pi^{2}(r-1)}{r} \int_{M}\left[c_{1}^{2}(E)-\frac{2 r}{r-1} c_{2}(E)\right] \\
& \times \frac{\omega^{n-2}}{(n-2) !} .
\end{aligned}
$$

As a corollary, if a holomorphic bundle $E$ admits a Hermitian-Yang-Mills connection, then it must satisfy the following Chern number inequality,

$$
\int_{M} c_{1}^{2}(E) \omega^{n-2} \leq \frac{2 r}{r-1} \int_{M} c_{2}(E) \omega^{n-2} .
$$

Furthermore, if the equality sign holds, then $F_{E}=\frac{1}{r} \operatorname{Tr}\left(F_{E}\right) I$, i.e. a projectively flat connection and thus $D_{E}$ corresponds to a homomorphism $\rho$ : $\pi_{1}(M) \rightarrow \mathbb{P U}(r)$.

A necessary condition for the existence of Hermitian-Yang-Mills connection is $E$ being a Mumford polystable bundle, i.e. a direct sum of Mumford stable bundle. Recall that $E$ is Mumford stable if every nontrivial coherent subsheaf $S$ in $E$ satisfies the following slope inequality,

$$
\mu_{S}<\mu_{E} .
$$

The celebrated theorem of Donaldson [36], Uhlenbeck and Yau [125] says that the converse is also true. Namely a holomorphic bundle $E$ admits a Hermitian Yang-Mills connection if and only if $E$ is a Mumford polystable bundle.

Mumford stability was introduced to construct projective moduli space of holomorphic bundles over Riemann surfaces via geometric invariant theory (GIT). The correct notion of stability in higher dimensions is the Gieseker stability which replaces the slope inequality by the normalized Hilbert polynomial inequality,

$$
\frac{1}{r k(S)} \chi\left(M, S \otimes L^{\otimes k}\right)<\frac{1}{r k(E)} \chi\left(M, E \otimes L^{\otimes k}\right)
$$

for sufficiently large $k$. Here $L$ is a line bundle over $M$ with $c_{1}(L)$ represented by $\omega$ and $\chi\left(M, E \otimes L^{\otimes k}\right)$ is given by

$\chi\left(M, E \otimes L^{\otimes k}\right)=\sum_{q=0}^{n}(-1)^{q} \operatorname{dim} H^{q}\left(M, E \otimes L^{\otimes k}\right)=\int_{M} \operatorname{Tr}_{E} e^{\frac{i}{2 \pi} F_{E}+k \omega} T d_{M}$,

by the Riemann-Roch formula and the Chern-Weil theory. Notice that the dominating terms for large $k$ is given by the slope. In $[\mathbf{8 8}]$ the author showed 
that Gieseker polystability is a necessary and sufficient condition for the existence of a bounded solution to the following equation,

$$
\left(e^{\frac{i}{2 \pi} F_{E}+k \omega} T d_{M}\right)^{[2 n]}=\frac{\chi\left(M, E \otimes L^{\otimes k}\right)}{r k(E)} \frac{\omega^{n}}{n !} I_{E},
$$

for sufficiently large $k$ on any sufficiently smooth holomorphic bundle $E$. The relationship with the symplectic geometry will be discussed in section 5.3.

Curvature for Kähler metrics. When $h=g$ is a Hermitian metric on the tangent bundle $E=T_{M}$, then the Kählerian condition for $g$ is equivalent to $D_{E}$ being torsion free. Yet another equivalent definition of $g$ being a Kähler metric is the existence of holomorphic normal coordinate, i.e. given any point $p_{0} \in M$, there exists a local holomorphic coordinate $z^{j}=x^{j}+i y^{j}$ 's such that for any nearby point $p$, we have

$$
g_{i \bar{j}}(p)=\delta_{i j}+O\left(\left|p-p_{0}\right|^{2}\right) .
$$

The symmetries for the curvature tensor $R m$ of a Kähler metric are richer than the Riemannian case. First we note that the inclusion $u(n) \subset$ $o(2 n)$ corresponds to $\left(V^{1,1}\right)_{\mathbb{R}} \subset \Lambda^{2} V_{\mathbb{R}}$ for any Hermitian vector space $V$. This implies that $R m_{i \bar{j} k \bar{l}}$ is Hermitian symmetric with respect to $i \bar{j}$ and also to $k \bar{l}$ for any complex coordinates $z^{j}=x^{j}+i y^{j}$ 's. Second the Ricci tensor $R c$ being Hermitian symmetric means that $R c \in u(n) \cong\left(\Lambda^{1,1} T_{M}^{*}\right)_{\mathbb{R}}$ and the corresponding $(1,1)$-form $R c(J \cdot, \cdot)$ will again be denoted as $R c_{i \bar{j}}$. Explicitly we have

$$
\begin{aligned}
R c_{i \bar{j}} & =g^{k \bar{l}} R m_{i \bar{j} k \bar{l}} \\
& =\frac{\partial}{\partial z^{i}} \frac{\partial}{\partial \bar{z}^{j}} \log \operatorname{det}\left(g_{k \bar{l}}\right) .
\end{aligned}
$$

In particular, the first Chern class of $M$ (modulo torsion) is represented by the Ricci form,

$$
c_{1}(M)_{\mathbb{R}}=\frac{i}{2 \pi}[R c]
$$

The scalar curvature is given by $R=g^{i \bar{j}} R c_{i \bar{j}}=\Delta \log \operatorname{det}\left(g_{k \bar{l}}\right)$.

Recall in the Riemannian case that when $R c_{M} \geq 0$, harmonic one forms are parallel and they must be zero if $R c_{M}>0$. In the Kähler case, holomorphic $p$-forms are harmonic. When $M$ has $R c_{M} \geq 0$, they are parallel and zero if $R c_{M}>0$, i.e. $M$ is Fano.

If $R m=\lambda I$ as an endomorphism of $u(n)$, then the universal cover of $M$ must be either the complex projective space $\mathbb{C P}^{n}$, the complex ball or $\mathbb{C}^{n}$ according to $\lambda$ being positive, negative or zero. These are called complex space forms.

If $R c=\lambda \omega$, i.e. $M$ is a Kähler-Einstein manifold, then first of all $c_{1}(M)_{\mathbb{R}}$ must be represented by a multiple of some Kähler form $\omega_{0}$. Unless $c_{1}(M)_{\mathbb{R}}=$ 0 , the canonical line bundle $K_{M}=\Lambda^{n} T_{M}^{*}$ or its inverse is positive and 
therefore $M$ is a projective manifold by the Kodaira's embedding theorem. If we write $R c\left(\omega_{0}\right)=\lambda \omega_{0}+i \bar{\partial} \partial f$ and $\omega=\omega_{0}+i \bar{\partial} \partial \phi$ for some smooth functions $f$ and $\phi$ on $M$ with average one, then the Kähler-Einstein equation is reduced to the following fully nonlinear second order elliptic equation, called the complex Monge-Ampère equation,

$$
\operatorname{det}\left(g_{i \bar{j}}+\frac{\partial^{2} \phi}{\partial z^{i} \partial \bar{z}^{j}}\right)=e^{-\lambda \phi+f} \operatorname{det}\left(g_{i \bar{j}}\right) .
$$

When $c_{1}(M)_{\mathbb{R}}=0$, Yau $[\mathbf{1 3 7}]$ solved this equation and proved that there is a unique metric in every Kähler class with zero Ricci curvature, $R c=0$. In fact, Yau's theorem also solved a conjecture of Calabi which says that every $(1,1)$-form representing $c_{1}(M)_{\mathbb{R}}$ can be represented uniquely as the Ricci form of a metric in any Kähler class on any compact Kähler manifold $M$.

As a corollary of Yau's theorem, $c_{1}(M)_{\mathbb{R}}=0$ implies that

$$
c_{2}(M)[\omega]^{n-2} \geq 0,
$$

for any Kähler class $[\omega]$ and the equality sign holds if and only if the universal cover of $M$ is $\mathbb{C}^{n}$. Zero Ricci curvature means that the canonical line bundle $K_{M}=\Lambda^{n} T_{M}^{*}$ is a flat line bundle. Suppose that $c_{1}(M)=0$ as an integral class, then $K_{M}$ is indeed trivial and its covariant constant section defines a holomorphic volume form on $M$. Thus the holonomy group of $M$ is reduced to $S U(n)$ and such a manifold is called a Calabi-Yau manifold and plays a very important role in string theory.

When $c_{1}(M)_{\mathbb{R}}<0$, Aubin and Yau solved the Monge-Ampère equation, thus proving that there is a unique Kähler-Einstein metric in the Kähler class $-c_{1}(M)_{\mathbb{R}}$. The Chern number inequality becomes

$$
(-1)^{n} c_{1}^{n}(M) \leq \frac{2(n+1)}{n}(-1)^{n} c_{2}(M) c_{1}^{n-2}(M) .
$$

When the equality sign holds, then the universal cover of $M$ must be the complex hyperbolic ball.

When $c_{1}(M)_{\mathbb{R}}>0, M$ is called a Fano manifold and there are nontrivial obstructions to the existence of Kähler-Einstein metrics, for instance the Futaki invariant. Yau conjectured that there should be a notion of stability which relates to the existence of such a canonical metric. This problem has been studied by many mathematicians including Donaldson, Mabuchi, Phong, Tian and others. Donaldson showed that this relationship between stability and the existence of canonical metrics should continue to hold true for constant scalar curvature Kähler metrics, i.e. $R=$ const.

5.3. Kähler geometry-symplectic aspects. Recall that the Kähler form $\omega(u, v)=g(J u, v)$ defines a closed non-degenerate two form, i.e. a symplectic form. On the classical level, symplectic geometry is a much more linear theory than the complex geometry (section 5.3). On the quantum level, it becomes a very rich and challenging subject which includes 
the theories of Gromov-Witten invariants and the Fukaya-Floer category. The mirror symmetry conjecture says roughly that complex geometry and quantum symplectic geometry should be equivalent to each other.

Basic symplectic geometry. Let us start by reviewing some aspects of the classical symplectic geometry. First the Darboux lemma says that every symplectic manifold is locally standard which is $\mathbb{R}^{2 n}$ with

$$
\omega_{0}=\sum_{j=1}^{n} d x^{j} \wedge d y_{j}
$$

We can view this as the cotangent bundle $T^{*} \mathbb{R}^{n}$ of $\mathbb{R}^{n}$ with coordinates $x^{j}$ 's and dual coordinates $y_{j}$ 's along fibers. In fact $\omega_{0}$ defines a canonical symplectic structure on the cotangent bundle $M=T^{*} X$ of any manifold $X$.

A vector field $v$ on $M$ preserves the symplectic form $\omega$ if and only if $\iota_{v} \omega$ is a closed one form. If $\iota_{v} \omega=d f$ is exact, then $v$ is called a Hamiltonian vector field. Moser's lemma says that if $\omega_{t}$ is an one parameter family of symplectic forms in $M$ representing the same cohomology class, then all these $\left(M, \omega_{t}\right)$ 's are symplectomorphic to each other. Thus the moduli space of symplectic structures on $M$ is locally isomorphic to $H^{2}(M, \mathbb{R})$. One can also include $B$-fields $B$ on $M$ and such that the moduli space of symplectic structures with B-fields $B+i \omega$ is a complex space which is locally isomorphic to $H^{2}(M, \mathbb{C})$. This concept is originated in string theory and important in mirror symmetry.

The natural class of submanifolds in $(M, \omega)$ consists of Lagrangian submanifolds $L$ which are $n$-dimensional submanifolds in $M$ satisfying $\left.\omega\right|_{L}=0$. For instance the zero section and every fiber in the cotangent bundle $T^{*} X$ are Lagrangian submanifolds. In general, if we regard a section $L$ in $T^{*} X$ as the graph of an one form $\phi \in \Omega^{1}(X)$, then $L$ is a Lagrangian submanifold if and only if $\phi$ is a closed form, i.e. $d \phi=0$. Indeed a neighborhood of any Lagrangian submanifold $L$ is always symplectically equivalent to the cotangent bundle $T^{*} L$ and therefore the moduli space of Lagrangian submanifolds $L$ in $M$ modulo Hamiltonian equivalences is locally given by $H^{1}(L, \mathbb{R})$. If we consider the moduli space of $A$-cycles $\left(L, D_{E}\right)$, i.e. $L$ is a Lagrangian submanifold in $M$ and $D_{E}$ is a flat $U(1)$-connection over $L$, then it is again a complex manifold and locally isomorphic to $H^{1}(L, \mathbb{C})$.

Lagrangian fibrations. A Lagrangian fibration $\pi: M \rightarrow B$ is called a (singular) real polarization on $M$, or an integrable system. It plays an important role in geometric quantization of the symplectic manifold $M$. Another standard way to obtain geometric quantization is to equip $M$ with a complex structure such that $\omega$ is the corresponding Kähler form, this is called a complex polarization.

Away from singular fibers, we have a surjective bundle homomorphism $T M \rightarrow \pi^{*} T B$ over $M$ and the kernel is called the vertical tangent bundle $T_{\text {vert }} M$. For Lagrangian fibration, we have

$$
T_{\text {vert }} M \cong \pi^{*} T^{*} B
$$


Thus we have $n$ commuting vector fields along fibers which are linearly independent at every point. This determines canonical affine structures on smooth fibers. When $\pi$ is proper, i.e. fibers are compact, then fibers are tori $T_{x}^{*} B / \Lambda_{x}$. This gives a lattice subbundle $\Lambda$ in $T^{*} B$ over $B$ outside the discriminant locus $\operatorname{Disc}(\pi)$. As a result, the base $B \backslash \operatorname{Disc}(\pi)$ also has a $G L(n, \mathbb{Z}) \times \mathbb{Z}^{n}$-affine structure.

Toric varieties $\mathbb{P}_{\Delta}$ are examples of symplectic manifolds with Lagrangian fibrations in which the fibers are orbits of an Hamiltonian torus action (see below) and the base is a convex polytope $\Delta$. The simplest compact toric varieties are certainly complex projective spaces $\mathbb{C P}^{n+1}$.

Hamiltonian action and symplectic reduction. Suppose $(M, \omega)$ is a symplectic manifold with a Hamiltonian action by a compact Lie group $G$ with moment map

$$
\mu: M \rightarrow(L i e G)^{*} .
$$

We recall that a moment map $\mu$ is a $G$-equivariant map such that for any $v \in \operatorname{Lie}(G)$ and $v^{\#}$ the vector field on $M$ that $v$ generates, then $v^{\#}$ is the Hamiltonian vector field for the function $x \rightarrow \mu(x)(v)$, i.e.

$$
\iota_{v} \# \omega=d(\mu(v)) .
$$

If a $2 n$-dimensional symplectic manifold $M$ has an effective Hamiltonian $T^{k}$-action, then $k$ is at most $n$. When $k=n$, the moment map

$$
\mu: M \rightarrow \mathbb{R}^{n}
$$

is a Lagrangian fibration on $M$ and the image is convex polytope $\Delta$ in $\mathbb{R}^{n}$. Such a $M$ is called a toric variety and its geometry is completely dictated by the polytope $\Delta$. For instance, when $\Delta$ is the standard simplex in $\mathbb{R}^{n}, M$ is the complex projective space $\mathbb{C P}^{n}$ with the toric action is induced from $T^{n} \subset\left(\mathbb{C}^{\times}\right)^{n} \subset \mathbb{P}^{n}$.

When $M$ has a Hamiltonian $G$-action, there is a procedure to divide out the symmetry to produce another (possibly singular) symplectic manifold

$$
M / / G=\mu^{-1}(0) / G
$$

called the symplectic quotient or symplectic reduction. We can also replace 0 by other coadjoint orbit.

We assume that $(M, \omega)$ is a Kähler manifold with $\omega$ being defined over $\mathbb{Z}$, thus $[\omega]=c_{1}(L)$ for a positive line bundle $L$. Suppose that the complexification $G^{\mathbb{C}}$ of $G$ acts holomorphically on $M$ and the action can be lifted to $L$. We can apply the Geometric Invariant Theory, developed by Mumford, to construct a quotient space $M / G^{\mathbb{C}}$ within the category of algebraic varieties. In this construction, one needs to remove unstable points in $M$ to ensure that the quotient space is Hausdorff. Kempf-Ness showed that this complex algebraic approach and the symplectic approach of taking quotient are equivalent to each other,

$$
M / G^{\mathbb{C}} \cong M / / G .
$$


This identification is particularly fruitful in many infinite dimensional settings as a guiding principle.

Symplectic geometry and gauge theory. Given a Hermitian complex vector bundle $E$ over a symplectic manifold $(M, \omega)$, the space $\mathcal{A}(E)$ of unitary connections on $E$ has a natural symplectic form $\Omega$ defined as follows: The tangent space of $\mathcal{A}(E)$ at any connection $D_{A}$ can be identified as $\Omega^{1}(M, a d(E))$. Given any tangent vectors $B$ and $C$, we define

$$
\Omega\left(D_{A}\right)(B, C)=\int_{M} \operatorname{Tr}_{E} B \wedge C \wedge \frac{\omega^{n-1}}{(n-1) !} .
$$

The action on $\mathcal{A}(E)$ by the group $\mathcal{G}(E)$ of gauge transformations of $E$ preserves $\Omega$ and its moment map is given by

$$
\begin{aligned}
\mu & : \mathcal{A}(E) \rightarrow \Omega^{2 n}(M, a d(E)) \\
\mu\left(D_{A}\right) & =F_{E} \wedge \frac{\omega^{n-1}}{(n-1) !} .
\end{aligned}
$$

When $M$ is a Riemann surface, both $\Omega$ and $\mu$ are independent of the symplectic form on $M$ and the symplectic quotient

$$
\mathcal{A}(E) / / \mathcal{G}(E)=\left\{D_{A}: F_{A}=0\right\} / \mathcal{G}(E),
$$

is the moduli space of flat connections on $E$, i.e.

$$
\operatorname{Hom}\left(\pi_{1}(M), U(r)\right) / U(r) \text {. }
$$

There are natural generalizations of this to other compact Lie groups. The infinite dimensional analog of the identification between GIT quotient and symplectic quotient suggests that every polystable holomorphic bundle over $M$ admits a unitary flat connection. This was proved by Narishima-Seshadra.

When $M$ is a Kähler manifold and $E$ is a holomorphic vector bundle over it, we can restrict our attention to the subset $\mathcal{A}^{\text {hol }}(E)$ consisting of those connections $D_{A}$ satisfying $F_{A}^{0,2}=0$, namely $\left(D_{A}\right)^{0,1}$ defines a holomorphic structure on $E$. In this case, the suggested isomorphism between $\mathcal{A}^{\text {hol }}(E) / / \mathcal{G}(E)$ and $\mathcal{A}^{\text {hol }}(E) / \mathcal{G}^{\mathbb{C}}(E)$ is the theorem of Donaldson [36] and Uhlenbeck-Yau [125] which says that every Mumford polystable holomorphic bundle admits a unique Hermitian-Yang-Mills connection.

However, in algebraic geometry, the correct stability condition is the Gieseker stability defined using the Hilbert polynomial,

$$
\chi\left(M, E \otimes L^{\otimes k}\right)=\int_{M} \operatorname{Tr}_{E}\left[e^{\frac{i}{2 \pi} F_{A}+k \omega I_{E}} \wedge T d_{M}\right]
$$

The analog of the equivalence between GIT quotient and the symplectic quotient was established in [88] for the existence of solutions to the following equations for large $k$,

$$
\left[e^{\frac{i}{2 \pi} F_{A}+k \omega I_{E}} \wedge T d_{M}\right]^{(n, n)}=\frac{\chi\left(M, E \otimes L^{\otimes k}\right)}{r} I_{E} \frac{\omega^{n}}{n !} .
$$


This equation is also a moment map equation for the $\mathcal{G}(E)$-action on $\mathcal{A}^{\text {hol }}$ $(E)$ with respect to the following nonconstant symplectic form $\Omega_{k}[\mathbf{8 9}]$ :

$$
\Omega_{k}\left(D_{A}\right)(B, C)=\int_{M} \operatorname{Tr}_{E}\left[e^{\frac{i}{2 \pi} F_{A}+k \omega I_{E}} \wedge B \wedge C\right]_{s y m} \wedge T d_{M} .
$$

The Gieseker stability is an asymptotic stability as $k$ goes to infinity. For each finite $k$, Donaldson studied the finite dimensional GIT/symplectic quotients equivalences and conjectured that Gieseker stable bundles should admit balanced metrics for large $k$ 's and they converge to the HermitianYang-Mills metrics if $E$ is also Mumford polystable. This problem was solved by Wang $[\mathbf{1 2 9}]$.

Space of Kähler forms. Donaldson and Semmes showed that the space of Kähler metrics in a fixed Kähler class on $M$ is an infinite dimensional symplectic manifold with a Hamiltonian action by Diff $(M)$. Furthermore, the moment map can be identified with the scalar curvature of a Kähler metric. Thus the GIT/symplectic quotients equivalences should relate the existence of constant scalar curvature Kähler metrics with GIT stability of the manifold $M$.

In the next section, we discuss symplectic geometry on the quantum level.

\subsection{Gromov-Witten theory.}

Gromov-Witten invariants. Given a complex structure $J$ on $M$, we study complex submanifolds $C$ in $M$, namely $T_{x} C$ is $J$-linear in $T_{x} M$ for any $x \in C$. This notion continues to make sense for any almost complex structure and such submanifolds $C$ are called $J$-pseudo holomorphic submanifolds, or simply J-holomorphic submanifolds. Recall that an almost complex structure is simply a complex structure on the tangent bundle $T_{M}$. If we choose a generic almost complex structure on $M$, then it admits no $J$-holomorphic submanifolds $C$ of $\operatorname{dim}_{\mathbb{C}} C \geq 2$, even locally, as the Cauchy-Riemann equation is an over-determined system of differential equations. However, there are always many $J$-holomorphic curves, at least locally. In order to count the number of such curves, it turns out that it is better to use the parametrized version, namely we consider the moduli space $\mathcal{M}^{\text {curve }}(M)$ of $J$-holomorphic maps from genus $g$ Riemannian surfaces $\Sigma$ to $M$,

$$
f: \Sigma \rightarrow M \text {. }
$$

When $M$ is equipped with a compatible symplectic structure $\omega$, then $\mathcal{M}^{\text {curve }}$ $(M)$ has a natural compactification $\overline{\mathcal{M}^{\text {curve }}(M)}$ by stable maps where the domain Riemann surface $\Sigma$ is allowed to have nodal singular points and $f$ is required to have only finite number of automorphisms. Recall that every $J$-holomorphic curve is calibrated by $\omega$, in particular, $f(\Sigma)$ always represents a nontrivial homology class $[f(\Sigma)] \in H_{2}(M, \mathbb{Z}) \backslash\{0\}$.

Infinitesimal deformations of $f$ are parametrized by $H^{0}\left(\Sigma, N_{f(\Sigma) / M}\right)$ (section 5.1). When $H^{1}\left(\Sigma, N_{f(\Sigma) / M}\right)=0$, then $f$ always has unobstructed 
deformations. In this case, $\mathcal{M}^{\text {curve }}(M)$ is smooth and its dimension is determined by the Riemann-Roch formula,

$$
\operatorname{dim}_{\mathbb{C}} \mathcal{M}^{\text {curve }}(M)=\int_{\Sigma} f^{*} c_{1}(M)+(g-1)(3-n) .
$$

In order to count the number of curves in $M$, we impose conditions to cut down the dimension of $\overline{\mathcal{M}^{\text {curve }}(M)}$. For instance, we can require $f(\Sigma)$ to pass through specific points in $M$. These define the Gromov-Witten invariants, or simply $G W$-invariants. These are invariants for the deformation class of symplectic forms on $M$. In particular, it is independent of the choice of compatible almost complex structures on $M$. Even when $\overline{\mathcal{M}^{\text {curve }}(M)}$ is singular, there is a theory using virtual fundamental class to define these invariants.

GW-invariants can be interpreted as the partition functions for the A-model TFT in the $N=2$ SUSY $\sigma$-model on the Kähler manifold $M$.

In general, GW-invariants are difficult to compute. When $\operatorname{dim}_{\mathbb{R}} M=4$, Taubes showed that GW-invariants with no constraints are equivalent to the SW-invariants. This result has far-reaching consequences in four dimensional symplectic geometry. When the symplectic manifold $(M, \omega)$ has a lot symmetries, for instance a toric variety, then GW-invariants can be computed in many instances via Bott localization, at least in the genus zero case. This method can be generalized to complex hypersurfaces of small degrees in Fano toric varieties as well. It was initiated by Kontsevich, motivated from the mirror symmetric conjecture for Calabi-Yau manifolds. The mirror theorem which computes the genus zero GW-invariants for quintic CY threefolds in terms of the variation of Hodge structures of its mirror manifold was proven by developing this approach by Givental [47] and Liu-Lian-Yau [105]. There are other approaches in determining GW-invariants, for example the YauZaslow argument for the number of rational curves on K3 surfaces (section 6.4) and various physical methods coming from dualities in string theory and M-theory.

The genus zero GW-invariants can be used to deform the cup product structure on the cohomology ring $H^{*}(M)$ and results in the quantum cohomology ring $Q H^{*}(M)$ for the symplectic manifold $(M, \omega)$. It is originated from the (closed) string theory. $Q H^{*}(M)$ can be formally interpreted as the middle dimensional cohomology ring of the free loop space $\mathcal{L} M$ via the Witten-Morse theory for the symplectic area functional

$$
\begin{gathered}
A: \mathcal{L} M \rightarrow \mathbb{R} \\
A(\gamma)=\int_{\gamma_{0}}^{\gamma_{1}} \omega .
\end{gathered}
$$

Here $\gamma_{t}$ is an one-parameter family of loops in $M$ connecting $\gamma=\gamma_{1}$ to a background $\gamma_{0}$. Here $\int_{\gamma_{0}}^{\gamma_{1}}$ means the integration over the two dimensional surface $\coprod_{t \in[0,1]} \gamma_{t}$ spanned by the path of loops. Critical points of $A(\gamma)$ are 
constant loops in $M$, thus $M$ can be viewed as the critical set of $A$ inside $\mathcal{L} M$. After choosing a compatible metric on $M$, gradient flow lines in $\mathcal{L} M$ correspond to $J$-holomorphic cylinders in $M$.

Floer theory of Lagrangian intersections. In open string theory, the boundaries of a string, namely a path $\gamma(t)$ in $M$, lies on Lagrangian submanifolds $L_{i}$ in $M$, i.e. $\gamma(0) \in L_{0}$ and $\gamma(1) \in L_{1}$. Similarly, we consider the symplectic area functional $A$ on the space $\mathcal{L}_{L_{0} \rightarrow L_{1}} M$ of all such paths. Critical points of $A$ are constant paths and therefore correspond to intersection points in $L_{0} \cap L_{1}$ and gradient flow lines of $A$ are holomorphic strips between $L_{i}$ 's joining two intersection points. Floer and others developed the Witten-Morse theory in this setting and defined Floer cohomology groups $H F_{\text {Lagr }}^{M}\left(L_{0}, L_{1}\right)$ for Lagrangian intersections. Fukaya and others [45] extended these structures and defined the Fukaya category Fuk $(M)$, which is conjecturally dual to the derived category of coherent sheaves under mirror symmetry.

\section{Calabi-Yau geometry}

6.1. Calabi-Yau manifolds. A Kähler manifold $M$ is called a CalabiYau manifold if it admits a parallel holomorphic volume form $\Omega \in \Omega^{n, 0}(M)$, i.e.

$$
\begin{aligned}
\nabla \Omega & =0 \\
(2 i)^{-n}(-1)^{n(n+1) / 2} \Omega \wedge \bar{\Omega} & =v_{M},
\end{aligned}
$$

where $v_{M}=\omega^{n} / n$ ! is the Riemannian volume form and $n$ is the complex dimension of $M$. Equivalently, $M$ is a Riemannian manifold with holonomy group inside $S U(n)$. If $M$ is compact, then $\Omega$ being parallel can be replaced by holomorphicity, i.e. $\bar{\partial} \Omega=0$. The complex volume form $\Omega$ defines a $\mathbb{C}$ orientation on $M$ and it fits into the unified description of geometries of special holonomy in terms of normed division algebras (see section 10).

Recall that a volume form $v_{M}$ on $M$ defines a symplectic form on $\mathcal{K}_{\Sigma}(M)=\operatorname{Map}_{\text {emb }}(\Sigma, M) / \operatorname{Diff}(\Sigma)$ where $\operatorname{dim} \Sigma=\operatorname{dim} M-2$. One can also use the holomorphic volume form $\Omega$ to define a holomorphic symplectic form on the isotropic knot space $\mathcal{K}_{\Sigma}^{\mathbb{C}}(M)$ where $\operatorname{dim}_{\mathbb{R}} \Sigma=n-2$. Furthermore the special Lagrangian geometry of $M$ can be interpreted as the complex symplectic geometry of $\mathcal{K}_{\Sigma}^{\mathbb{C}}(M)$ (section 9.4 ).

Since the Ricci curvature of a Kähler metric is given by

$$
R c_{i \bar{j}}=-\frac{\partial^{2}}{\partial z^{i} \partial \bar{z}^{j}} \log \operatorname{det}\left(g_{k \bar{l}}\right),
$$

any Calabi-Yau manifold has zero Ricci curvature. In particular, its canonical line bundle $K_{M}$ is trivial and $c_{1}(M)=0$. By Yau's celebrated theorem [137], any compact Kähler manifold $M$ with $c_{1}(M)=0$ admits a unique Calabi-Yau metric in every Kähler class. Thus it is easy to identify which Kähler manifold admits a Calabi-Yau metric. Nevertheless, we still do not 
know how to write down explicitly any nontrivial Calabi-Yau metric on any compact Calabi-Yau manifold. For instance, any smooth projective hypersurface of degree $n+2$ in $\mathbb{C P}^{n+1}$ is a Calabi-Yau manifold. More generally, a hypersurface in a toric variety $X_{\Delta}$ representing the class $c_{1}\left(X_{\Delta}\right)$ is a (possibly singular) Calabi-Yau variety if and only if $\Delta$ is a reflexive polytope, i.e. both $\Delta$ and its polar dual polytope $\nabla$ are integral. This construction can be easily generalized to complete intersections in toric varieties and produces many examples of Calabi-Yau manifolds.

Even though we do not know any explicit Calabi-Yau metric on compact manifolds, there are many such examples on noncompact manifolds, including the total space of the canonical line bundle $K_{\mathbb{C P}^{n-1}}$ of $\mathbb{C P}^{n-1}$ and the cotangent bundle $T_{S^{n}}^{*}$ of the sphere. These metrics are found by utilizing the symmetries of these spaces to reduce the Monge-Ampère equation to an ODE.

Every holomorphic $p$-form in $H^{p, 0}(M)$ is parallel since $R c_{M}=0$ for a Calabi-Yau manifold $M$. The existence of such forms can usually be used to reduce the holonomy group of $M$ to a smaller subgroup. Using the deRham decomposition for holonomy groups, up to a finite cover, $M$ is a product of irreducible factors and they are (i) complex tori $\mathbb{C}^{n} / \Gamma$, (ii) irreducible hyperkähler manifolds, i.e. $h o l=S p(n / 2)$ or (iii) strict Calabi-Yau manifolds, i.e. $h o l=S U(n)$. Only complex tori have nontrivial holomorphic one forms and $h^{p, 0}\left(\mathbb{C}^{n} / \Gamma\right)=\left(\begin{array}{l}n \\ p\end{array}\right)$. Hyperkähler manifolds admit holomorphic symplectic forms, indeed $h^{2 l, 0}=1$ and $h^{2 l+1,0}=0$ for irreducible hyperkähler manifolds. We will discuss more about the hyperkähler geometry in section 11.1. When hol $=S U(n)$, the only nontrivial holomorphic $p$-form is the holomorphic volume form and therefore $h^{p, 0}=0$ for $1 \leq p \leq n-1$. By the Lefschetz hyperplane theorem, any Calabi-Yau hypersurface, or complete intersection, in a Fano toric variety is a strict Calabi-Yau manifold provided that $n \geq 2$.

Since $K_{M}$ is trivial for a Calabi-Yau manifold, we have

$$
H^{q}\left(M, T_{M}\right) \cong H^{q}\left(M, \Lambda^{n-1} T_{M}^{*}\right) \cong H^{n-1, q}(M) .
$$

In particular, the spaces of infinitesimal deformations and obstructions are given by $H^{n-1,1}(M)$ and $H^{n-1,2}(M)$ respectively. Tian [122] and Todorov [124] proved that the Kuranishi map $\kappa: H^{1}\left(M, T_{M}\right) \rightarrow H^{2}\left(M, T_{M}\right)$ is zero. Thus the moduli space of complex structures on $M$ is always smooth and of dimension $h^{n-1,1}(M)$. This moduli space has many nice properties, especially in complex dimension three (section 7 ).

\subsection{Special Lagrangian geometry.}

Special Lagrangian submanifolds. For Kähler manifolds, complex submanifolds are calibrated and therefore they are absolute minimizers for the volume functional. For Calabi-Yau manifolds, there is a natural class of Lagrangian submanifolds which are calibrated. Harvey and Lawson [59] found that for any given phase angle $\theta$, the differential form $\operatorname{Re}\left(e^{i \theta} \Omega\right) \in$ 
$\Omega^{n}(M)$ is a calibrated form. Furthermore a submanifold $L$ in $M$ is calibrated by $\operatorname{Re}\left(e^{i \theta} \Omega\right)$ if and only if

$$
\left.\omega\right|_{L}=0 \text { and }\left.\operatorname{Im}\left(e^{i \theta} \Omega\right)\right|_{L}=0 .
$$

Such a $L$ is called a special Lagrangian submanifold of phase $\theta$.

We remark that for any Lagrangian submanifold $L$ in $M$, the volume form $v_{L}$ for the induced metric satisfies

$$
v_{L}=\left.e^{i \theta(x)} \Omega\right|_{L}
$$

for some function $\theta(x): L \rightarrow \mathbb{R} / 2 \pi \mathbb{Z}$. It is the Hamiltonian function for the mean curvature vector field on $L$, i.e.

$$
\iota_{H} \omega=d \theta(x) .
$$

This implies that the mean curvature flow preserves the Lagrangian property and they satisfy the PDE

$$
\frac{\partial \theta}{\partial t}=\Delta \theta
$$

This is a non-linear equation as the Laplacian is defined with respect to the induced metric on $L$ which is changing in time. Lagrangian mean curvature flow in Calabi-Yau manifolds enjoys many nice properties and the same is true for hyperlagrangian mean curvature flow in hyperkähler manifolds (section 10.3).

Unless specified otherwise, we assume that $\theta=0$ for the calibrating form. When $M=\mathbb{C}^{n}$, a Lagrangian graph $\operatorname{Graph}(d \phi)$ over $\mathbb{R}^{n}$ is special if and only if $\phi(x)$ satisfies

$$
\operatorname{Im} \operatorname{det}\left(\delta_{j k}+i \frac{\partial^{2} \phi}{\partial x^{j} \partial x^{k}}\right)=0 .
$$

Examples of special Lagrangian submanifolds include the real locus of a Calabi-Yau manifold, complex Lagrangian submanifolds in a hyperkähler manifold (section 11.1). Many explicit examples of special Lagrangian submanifolds had been constructed in the noncompact setting by imposing symmetries to reduce the equation to an ODE. There are also various compact examples constructed using singular perturbation method to resolve singular special Lagrangians.

In section 9.5, we show that the isotropic knot space $\mathcal{K}_{\Sigma}^{\mathbb{C}}(M)$ is an infinite manifold with an $\mathbb{H}$-structure such that (i) every special Lagrangian submanifold $L$ of phase 0 in $M$ determines a $J$-complex Lagrangian submanifold $\mathcal{K}_{\Sigma}^{\mathbb{C}}(L)$ in $\mathcal{K}_{\Sigma}^{\mathbb{C}}(M)$ and (ii) certain special Lagrangian submanifold $L^{\prime}$ of phase $\pi / 2$ in $M$ determines a $J$-holomorphic curve in $\mathcal{K}_{\Sigma}^{\mathbb{C}}(M)$. Thus the Calabi-Yau geometry can be interpreted as the hyperkähler geometry in the isotropic knot space $\mathcal{K}_{\Sigma}^{\mathbb{C}}(M)$. 
Moduli of Special Lagrangian submanifolds and A-branes. Recall that given any Lagrangian submanifold $L$ in $M$, its neighborhood is isomorphic symplectically to $T^{*} L$ and nearby Lagrangian submanifolds are graphs of closed one forms on $L$. When $L$ is special, then its infinitesimal deformations are parametrized by harmonic one forms on L. McLean [108] proved that there is no obstruction to extend any infinitesimal deformation to a honest one, thus the moduli space $\mathcal{M}^{\text {SLag }}(M)$ of special Lagrangian submanifolds $L$ in $M$ is smooth with tangent space $H^{1}(L, \mathbb{R})$. Hitchin [61] defined analogs of the (multi-valued) Abel-Jacobi map,

$$
\begin{aligned}
p & : \mathcal{M}^{S L a g}(M) \\
p^{\prime}: \mathcal{M}^{S L a g}(M) & \rightarrow H^{1}\left(L_{0}, \mathbb{R}\right) \text { and }\left(L_{0}, \mathbb{R}\right)
\end{aligned}
$$

and showed that $\left(p, p^{\prime}\right)$ is a Lagrangian immersion of $\mathcal{M}^{S L a g}(M)$ into $T^{*}$ $\left(H^{1}\left(L_{0}, \mathbb{R}\right)\right) \cong H^{1}\left(L_{0}, \mathbb{R}\right) \times H^{n-1}\left(L_{0}, \mathbb{R}\right)$. To define $p$, we consider a path of special Lagrangian submanifolds $L_{t}$ together with a loop $\gamma_{t}$ in each $L_{t}$, then $p\left(L_{1}\right) \in H^{1}\left(L_{0}, \mathbb{R}\right) \cong \operatorname{Hom}\left(H_{1}\left(L_{0}, \mathbb{R}\right), \mathbb{R}\right)$ is given by

$$
p\left(L_{1}\right)\left(\gamma_{0}\right)=\int_{\gamma_{0}}^{\gamma_{1}} \omega .
$$

Similarly, $p^{\prime}$ is defined using $\operatorname{Im} \Omega$ in place of $\omega$.

Note that the moduli space of flat $U(1)$-connections over $L$ is naturally $H^{1}\left(L, S^{1}\right)=i H^{1}(L, \mathbb{R}) / H^{1}(L, \mathbb{Z})$. Thus we could include flat $U(1)$ connections over $L$ to complexify the moduli space of special Lagrangian submanifolds to the moduli space of $A$-branes $\mathcal{M}^{A \text {-brane }}(M)$. It has a natural symplectic structure with a Lagrangian fibration,

$$
H^{1}\left(L, S^{1}\right) \rightarrow \mathcal{M}^{A-\text { brane }}(M) \rightarrow \mathcal{M}^{S L a g}(M) .
$$

Hitchin described $\mathcal{M}^{A-\text { brane }}(M)$ using the symplectic reduction method as follows: Fix a $n$-dimensional volume manifold $\left(L, v_{L}\right)$, then the mapping space $\operatorname{Map}(L, M)$ has a natural $\operatorname{Diff}\left(L, v_{L}\right)$-invariant symplectic form $\omega_{\text {Map }}$ given by

$$
\omega_{M a p}(f)(X, Y)=\int_{L} \omega(X, Y) v_{L}
$$

where $X, Y \in T_{[f]} \operatorname{Map}(L, M) \cong \Gamma\left(L, f^{*} T_{M}\right)$. When $H^{1}(L)=0$, this action is Hamiltonian and the moment map $\mu$ is given by

$$
\mu(f)=[\alpha] \in \Omega^{1}(L) / d \Omega^{0}(L) \text { with } f^{*} \omega=d \alpha .
$$

Thus

$$
\begin{aligned}
\operatorname{Map}(L, M) / / \operatorname{Diff}\left(L, v_{L}\right) & =\mu^{-1}(0) / \operatorname{Diff}\left(L, v_{L}\right) \\
& \cong \mathcal{M}_{[L]}^{L a g}(M)
\end{aligned}
$$

those components containing $f(L)$ in the moduli space of Lagrangian submanifolds in $M$. 
We can also restrict our attention to the complex submanifold $M a p^{\Omega}$ $(L, M)$ in $\operatorname{Map}(L, M)$ consisting of those $f^{\prime}$ 's satisfying $f^{*} \Omega=v_{L}$. Then

$$
\operatorname{Map}^{\Omega}(L, M) / / \operatorname{Diff}\left(L, v_{L}\right) \cong \mathcal{M}_{[L]}^{S \operatorname{Lag}}(M) .
$$

However, this is a discrete set as special Lagrangian submanifolds $L$ with $H^{1}(L)=0$ are rigid. In general, when $H^{1}(L) \neq 0$, the above construction has a natural generalization with the symplectic quotient isomorphic to $\mathcal{M}_{[L]}^{A-\text { brane }}(M)$.

Analogous to representing every deRham cohomology class by a unique harmonic form, we expect that most Hamiltonian equivalent classes of Lagrangian submanifolds in Calabi-Yau manifolds admits a unique special Lagrangian representative. The uniqueness question was studied by Thomas and Yau [120]. The existence part is a hard analytic problem. Schoen and Wolfson studied it using a variational approach while Smoczyk, M.T. Wang and others studied it using the mean curvature flow. Notice that the mean curvature flow preserves the class of Lagrangian submanifolds and its stationary points are given by special Lagrangian submanifolds inside CalabiYau manifolds.

Special Lagrangian fibration is an important ingredient in the SYZ proposal to explain the mirror symmetry phenomenons. Since we do not know how to write down the Calabi-Yau metrics, it is in general very difficult to find such fibrations, with the exception of complex Lagrangian fibrations on hyperkähler manifolds. In the next section, we will explain how we attempt to construct special Lagrangian fibrations on Calabi-Yau hypersurfaces in $\mathbb{C P}^{n+1}$.

6.3. Mirror symmetry. Mirror symmetry is a duality transformation which interchanges symplectic geometry and complex geometry between mirror Calabi-Yau manifolds (see e.g. [30]).

Physical origin, a brief encounter. Mirror symmetry is originated from the physical studies of the superstring theory. The spacetime in superstring theory has dimension ten. In order to reduce to our usual four dimensional spacetime, we need to compactify six dimensions. Furthermore, this six dimensional internal space $X$ must be a compact Calabi-Yau threefold, possibly coupled with a $E_{8} \times E_{8}$-bundle over it depending on the types of string theory under considerations.

There are particular topological sectors of this string theory, called the $A$-model and the $B$-model. From a mathematical point of view, they correspond to the symplectic geometry and the complex geometry of $X$.

Motivated from physical considerations, Greene and Plesser predicted that there should be a conjugate theory in which the A-model and B-model switch to each other, at least near the large complex structure limit (abbrev. LCSL). The corresponding compactified Calabi-Yau threefold $Y$ is called 
the mirror manifold to $X$.

$$
\begin{aligned}
& \text { A-model on } X \\
& \text { (symplectic geometry) } \\
& \overleftrightarrow{\text { mirror symmetry }} \quad \begin{array}{c}
\text { B-model on } Y \\
\text { (complex geometry) }
\end{array}
\end{aligned}
$$

The simplest Calabi-Yau threefold is the zero locus of a degree five homogeneous polynomial $f$ in $\mathbb{C P}^{4}$, the quintic Calabi-Yau threefold. For example if we take

$$
f\left(z_{0}, z_{1}, \ldots, z_{4}\right)=z_{0}^{5}+z_{1}^{5}+\cdots+z_{4}^{5}+\psi\left(z_{0} z_{1} \cdots z_{4}\right)
$$

then $X=\{f=0\}$ is a smooth Calabi-Yau threefold, called the Fermat Calabi-Yau threefold, provided that $\psi$ is any complex number not equal to one.

Candelas et al [29] did a highly nontrivial calculation of this equivalence for the Fermat Calabi-Yau threefolds and showed physically that the number of rational curves of any degree in $X$ can be read off explicitly from the periods of $Y$. This is an astonishing discovery as it relates two very different but equally important subjects in algebraic geometry, namely the enumerative geometry of $X$ and the variation of complex structures of $Y$.

Mirror of A-cycles and B-cycles. In 1994 Kontsevich [76] proposed a more precise conjecture on this duality between symplectic and complex geometries, called the homological mirror symmetry (HMS): If $X$ and $Y$ are mirror manifolds to each other, then the Fukaya-Floer category of Lagrangian intersections in $X$ is equivalent to the bounded derived category of coherent sheaves on $Y$. HMS conjecture works for Calabi-Yau manifolds of any dimension. For K3 surfaces, this conjecture was verified by Seidel [115]. There are also generalizations of this duality for Fano manifolds and general type manifolds.

The mirror symmetry conjecture predicts that (special) Lagrangian submanifolds should behave like (Hermitian Yang-Mills) holomorphic vector bundles, modulo quantum effects. Thomas and Yau [120] formulated a very interesting conjecture on the existence of special Lagrangian submanifolds which is the mirror of the theorem of Donaldson, Uhlenbeck and Yau on the existence of Hermitian Yang-Mills connections.

$S Y Z$ proposal. Strominger, Yau and Zaslow proposed a resolution of mirror symmetry in their groundbreaking paper [116]. They conjectured that (i) mirror Calabi-Yau manifolds $X$ and $Y$ should admit special Lagrangian torus fibrations with sections in the large volume/complex structure limit;

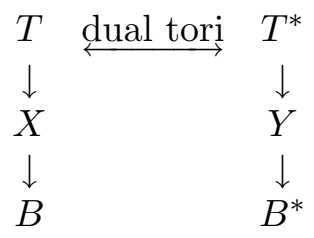

(ii) they are dual torus fibrations to each other; (iii) a fiberwise FourierMukai transformation along fibers interchanges the symplectic (resp. 
complex) geometry on $X$ with the complex (resp. symplectic) geometry on $Y$.

It roughly says that the mysterious duality is simply a Fourier transformation! The quantum corrections coming from holomorphic curves are higher Fourier modes.

A brief reasoning behind SYZ is as follows: From physical considerations, B-branes are complex submanifolds, or more generally derived equivalent classes of complexes of coherent sheaves, and A-branes are special Lagrangian submanifolds coupled with unitary flat bundles. As mirror symmetry should identify the complex geometry of $Y$ with the symplectic geometry of $X$, their moduli spaces of branes should be identified as well, at least at LCSL where quantum corrections had been suppressed. Since any space $Y$ is always the moduli space of points which are complex submanifolds, $Y$ should also be the moduli space of certain A-branes in $X$. Furthermore the underlying Lagrangian submanifolds $L$ of these A-branes should cover $X$ everywhere once, just like what points in $Y$ did. Since deformations of $L$ are parametrized by $H^{1}(L, \mathbb{R})$, we must have $\operatorname{dim} H^{1}(L, \mathbb{R})=n$ and $X$ should admit a special Lagrangian torus fibration

$$
T \rightarrow X \stackrel{\pi}{\rightarrow} B
$$

When we consider the complex submanifold which is $Y$ itself, the moduli space is a single point and the corresponding A-brane in $X$ would be a rigid special Lagrangian in $X$. Since $\int_{Y}[Y] \cup[p t]=1$, this rigid special Lagrangian submanifold should be a section to the above special Lagrangian fibration on $X$.

Next, given any torus fiber $T$ in $X$, its dual torus $T^{*}$ parametrizes flat $U$ (1)-bundles over $T$, namely A-branes in $X$ with support $T$. Under mirror symmetry, this $T^{*}$ also parametrizes corresponding B-branes in $Y$, which are points in $Y$. Thus $T^{*}$ is a subspace in $Y$ and therefore $Y$ also has a torus fibration by such $T^{*}$ 's.

$$
T^{*} \rightarrow Y \stackrel{\pi}{\rightarrow} B^{*}
$$

One can further argue that these two are dual special Lagrangian torus fibrations.

Besides giving dual fibrations on mirror manifolds $X$ and $Y$, we have a transformation between special Lagrangian fibers in $X$ with zero dimensional complex submanifolds in $Y$, namely points. This is a special case of a fiberwise Fourier-Mukai transformation. For more general special Lagrangian submanifolds in $X$, say a section to the above fibration, then the intersection point of it with any fiber $T$ would determine a flat $U(1)$ connection on $T^{*}$ because $\left(T^{*}\right)^{*}=T$. By patching them for various fibers $T$, we obtain a $U(1)$ connection on the whole manifold $Y$. One expects that this determines a holomorphic line bundle on $Y$ which is the mirror to the section 
in $X$. This was verified in $[\mathbf{1 0 1}]$ in the semiflat case. We call this transformation between the symplectic geometry of $X$ and the complex geometry of $Y$ the $S Y Z$ mirror transformation.

The SYZ transformation was generalized to the mirror symmetry for local Calabi-Yau manifolds by Leung-Vafa [97]. In [66], Hori-Vafa gave a physical proof of the mirror symmetry using the SYZ proposal.

Special Lagrangian fibrations. It is difficult to find special Lagrangian fibrations on compact Calabi-Yau manifolds as we do not know their metrics well. For noncompact Calabi-Yau manifolds, there are examples with explicit Calabi-Yau metrics. Most of these examples also admit explicit special Lagrangian fibrations. For instance,

$$
\begin{aligned}
\pi & : \mathbb{C}^{3} \rightarrow \mathbb{R}^{3} \\
\pi\left(z_{1}, z_{2}, z_{3}\right) & =\left(\left|z_{1}\right|^{2}-\left|z_{2}\right|^{2},\left|z_{2}\right|^{2}-\left|z_{3}\right|^{2}, \operatorname{Im} z_{1} z_{2} z_{3}\right)
\end{aligned}
$$

is a special Lagrangian fibration on $\mathbb{C}^{3}$ with generic fibers $T^{2} \times \mathbb{R}$ topologically.

In the following, we explain how we expect special Lagrangian fibrations should appear for hypersurfaces. Suppose that $M$ is a degree $n+2 \mathrm{CY}$ hypersurface in $\mathbb{C P}^{n+1}$

$$
M=\left\{f\left(z_{0}, z_{1}, \ldots, z_{n+1}\right)=0\right\} .
$$

The most singular one is given by the union of coordinate hyperplanes, namely

$$
M_{\infty}=\left\{z_{0} z_{1} \cdots z_{n+1}=0\right\} \subset \mathbb{C P}^{n+1} .
$$

For the family of Calabi-Yau manifolds $M_{t}$ defined by

$$
f\left(z_{0}, z_{1}, \ldots, z_{n+1}\right)+t \cdot z_{0} z_{1} \cdots z_{n+1}=0,
$$

the limit as $t$ goes to infinity is called the large complex structure limit (abbrev. LCSL). It can be characterized in terms of the period and it is also called the maximal unipotent monodromy limit $[\mathbf{2 9}]$. Notice that the smooth part $M_{\infty} \backslash \operatorname{Sing}\left(M_{\infty}\right)$ is a union of $\left(\mathbb{C}^{\times}\right)^{n}=T^{n} \times \mathbb{R}^{n}$ and one expects that these $T^{n}$-fibration can be perturbed and extended to give a special Lagrangian fibration on $M_{t}$ for $t$ large. Without the special condition, this approach was carried out by Gross, Mikhalkin, Ruan and Zharkov. However the question of whether one can make the Lagrangian fibrations on $X$ special is a much more delicate question as we do not understand the behavior of the Calabi-Yau metrics, whose existences are asserted by the celebrated theorem of Yau [137].

This approach can be generalized to Calabi-Yau hypersurfaces $X$ in any Fano toric variety $\mathbb{P}_{\Delta}$. Furthermore, their mirror manifolds $Y$ are Calabi-Yau hypersurfaces in another Fano toric variety $\mathbb{P}_{\nabla}$ whose defining polytope is the polar dual to $\Delta$. Thus we can see that the Lagrangian fibration structures on $X$ and $Y$ should be given by dual tori, at least away from singular fibers. 
The situation is quite different for Calabi-Yau twofolds, namely K3 surfaces, or more generally for hyperkähler manifolds. In this case, the CalabiYau metric on $X$ is Kähler with respect to three complex structures $I, J$ and $K$. When $X$ admits a $J$-holomorphic Lagrangian fibration, then this fibration is a special Lagrangian fibration with respect to the Kähler metric $\omega_{I}$, as well as $\omega_{K}$. Furthermore, SYZ also predicts that mirror symmetry is merely a twistor rotation from $I$ to $K$ in this case. For K3 surfaces, there are plenty of elliptic fibrations and they are automatically complex Lagrangian fibrations because of their low dimension. Furthermore Gross-Wilson [57] described the Calabi-Yau metrics for generic elliptic K3 surfaces by using the singular perturbation method. They used model metrics which were constructed by Greene, Shapere, Vafa and Yau [51] away from singular fibers and by Ooguri-Vafa [111] near singular fibers.

Recall that the base space $B$ of any compact Lagrangian fibration

$$
T \rightarrow X \stackrel{\pi}{\rightarrow} B
$$

admits a canonical integral affine structure, possibly with singularities. This affine structure will dictate the Calabi-Yau geometry at the large complex structure limit.

Outside the preimage of the singular set of $B$, the total space $X$ is given by the quotient of the cotangent bundle $T^{*} B$ by a lattice subbundle symplectically. In order to understand the A-model on $X$, we need to be able to describe rational curves and holomorphic disks on $X$ in terms of the affine structure on $B$. There has been much progress on this by the work of Fukaya, Kontsevich-Soibelman, Siebert-Gross and others. Here tropical geometry plays an important role.

The tangent bundle $T B$ of any affine manifold $B$ admits a canonical complex structure away from its singularities. Kontsevich and Soibelman [77], Gross and Siebert [55] described how to deform this complex structure at the large complex structure limit to nearby complex structures. Recall that the physical calculations of Candelas et al [29] showed that the variation of their Hodge structures should determine the Gromov-Witten invariants of rational curves of the mirror manifold. This important formula was later proven by Givental, Lian, Liu and Yau via a clever computation of Gromov-Witten invariants using localization method. The above program will eventually give a mathematical explanation of this phenomenon.

Explicit SYZ mirror transformation. Leung-Yau-Zaslow [101], [94] used the SYZ transformation to verify various correspondences between symplectic geometry and complex geometry between semi-flat Calabi-Yau manifolds. In this situation, there is no quantum corrections from instantons, namely rational curves or holomorphic disks. To include quantum corrections in the SYZ transformation for Calabi-Yau manifolds is a much more difficult problem. However in the Fano case, there are recent results on 
applying the SYZ transformation with quantum corrections by Auroux [8], Chan-Leung [31][32].

In order to understand the mirror transformation, we look at the simplest example, namely $\mathbb{C}^{n}$. If we view $\mathbb{C}^{n}$ as a tangent bundle over $B \cong \mathbb{R}^{n}$,

$$
M=T B
$$

then it has a canonical complex structure with holomorphic volume form

$$
\Omega_{M}=d z^{1} \wedge d z^{2} \wedge \cdots \wedge d z^{n} .
$$

If we view $\mathbb{C}^{n}$ as a cotangent bundle of $B \cong \mathbb{R}^{n}$,

$$
W=T^{*} B
$$

then it has a canonical symplectic form

$$
\omega_{W}=\sum d x^{j} \wedge d y_{j}
$$

Every linear subspace $P$ in $B$ determines a complex subspace $T P$ in $M$ as well as a Lagrangian subspace $N_{P / B}^{*}$ in $W$. They are fiberwise dual to each other with respect to the natural projections of $T B$ and $T^{*} B$ to $B$. Any Lagrangian section $L$ in $W$ is the graph of a closed one form $\eta$ on $B$. Let $D_{A}=d+i \alpha$ be a flat $U(1)$-connection over $L$, then $\bar{\partial}_{E}=\bar{\partial}+i \alpha+\beta$ defines a new holomorphic structure on the topologically trivial complex line bundle over $M$. These are the simplest Fourier-Mukai transformations, or the mirror transformations.

Any Riemannian metric $g$ on $B \cong \mathbb{R}^{n}$ induces compatible Riemannian metric $g_{M}$ and $g_{W}$ on (co)tangent bundles $M$ and $W$. Suppose $g$ is a Hessian metric, i.e. there is a convex function $\phi: B \rightarrow \mathbb{R}$ such that

$$
g_{i j}=\frac{\partial^{2} \phi}{\partial x^{i} \partial x_{j}} .
$$

Then $g_{M}$ is a CY metric on $M=T B$ if and only if $\phi$ satisfies the real MongeAmpère equation

$$
\operatorname{det}\left(\frac{\partial^{2} \phi}{\partial x^{i} \partial x^{j}}\right)=1
$$

On the other hand, $g_{W}$ is a CY metric on $W=T^{*} B$ if and only if the Legendre transformations $\psi$ of $\phi$ satisfies the real Monge-Ampère equation

$$
\operatorname{det}\left(\frac{\partial^{2} \psi}{\partial x_{i} \partial x_{j}}\right)=1 \text {. }
$$

We recall that the Legendre transformation of a convex function $\phi: B \rightarrow \mathbb{R}$ is another convex function $\psi: B^{*} \rightarrow \mathbb{R}$ satisfying

$$
\phi+\psi=\sum x_{j} x^{j}
$$


where the dual affine spaces $B$ and $B^{*}$ are identified under the map

$$
\begin{aligned}
B & \rightarrow B^{*} \\
x_{j}\left(x^{1}, \ldots, x^{n}\right) & =\frac{\partial \phi}{\partial x^{j}} .
\end{aligned}
$$

From above discussions, it is not surprising that the mirror symmetry transformation should be a fiberwise FM transform coupled with a Legendre transform along base directions.

In simple situations, namely semi-flat CY manifolds $[\mathbf{1 0 1}][\mathbf{9 4}]$, or Fano toric manifolds [32], explicit mirror transformations can be constructed to explain various dualities between complex and symplectic geometries. However, the general situation is still far from having a complete understanding, despite recent progress by Gross-Siebert [56].

\subsection{K3 surfaces.}

K3 surfaces as one dimension $\mathbb{H}$-manifolds. In this section, we study two dimensional Calabi-Yau manifolds $M$ in greater details. Since $S U(2)=$ $S p(1)$, a Calabi-Yau surface is the same as an one $\mathbb{H}$-dimensional hyperkähler manifold (section 10). This is similar to the fact that every oriented surface is a complex curve because of the isomorphism $S O(2)=U(1)$. The volume form on a Riemann surface is always a (Kähler) symplectic form. Similarly, the holomorphic volume form $\Omega_{J} \in \Omega^{2,0}(M)$ on a Calabi-Yau surface is always a holomorphic symplectic form. We decompose $\Omega_{J}$ into real and imaginary parts,

$$
\Omega_{J}=\omega_{I}-i \omega_{K}
$$

and we define $I$ and $K$ by

$$
\omega_{I}(u, v)=g(I u, v) \quad \text { and } \quad \omega_{K}(u, v)=g(K u, v) .
$$

Then just like the original complex structure $J$ on $M$, both $I$ and $K$ are orthogonal complex structures on $M$ with Kähler forms $\omega_{I}$ and $\omega_{K}$ respectively. Furthermore they satisfy the Hamilton relation

$$
I^{2}=J^{2}=K^{2}=I J K=-i d .
$$

This gives an explicit description of the $\mathbb{H}$-structure on any Calabi-Yau surface. See section 11.1 for more discussions on hyperkähler manifolds.

There are only two classes of compact Calabi-Yau surfaces, namely complex tori $\mathbb{C}^{2} / \Lambda$ and $\mathrm{K} 3$ surfaces.

A $K 3$ surface is a simply connected Kähler surface $M$ with $c_{1}(M)=0$. Siu and Todorov proved that the Kählerian property of $M$ is automatic. The cohomology group $H^{2}(M, \mathbb{Z})$ together with the quadratic form $q_{M}$ given by the intersection product is isomorphic to

$$
L_{K 3}=\left(-E_{8}\right) \oplus\left(-E_{8}\right) \oplus\left(\begin{array}{ll}
0 & 1 \\
1 & 0
\end{array}\right) \oplus\left(\begin{array}{ll}
0 & 1 \\
1 & 0
\end{array}\right) \oplus\left(\begin{array}{ll}
0 & 1 \\
1 & 0
\end{array}\right)
$$


where $E_{8}$ is the Cartan matrix for the exceptional Lie group $E_{8}$. In particular, $q_{M}$ has signature $(3,19)$.

Every smooth quartic surface in $\mathbb{C P}^{3}$ is a $\mathrm{K} 3$ surface. Also, given any complex torus $A=\mathbb{C}^{2} / \Lambda$, then $A / \mathbb{Z}_{2}$ has 16 ordinary double points. By blowing them up, we obtain a K3 surface. A particular nice class of K3 surfaces $M$ are elliptic K3 surfaces with sections.

Moduli of K3 surfaces. The moduli space of complex structures on K3 surfaces can be described in terms of the period map $\tau$. Given a K3 surface $M$ with holomorphic symplectic form $\Omega_{J}$, the span of $\left[\operatorname{Re} \Omega_{J}\right]$ and $\left[\operatorname{Im} \Omega_{J}\right]$ in $H^{2}(M, \mathbb{R})$ defines an element, called the period $\tau$, in the period domain $\mathcal{D}$,

$$
\mathcal{D}=S O^{+}(3,19) / S O(2) \times S O(1,19) .
$$

Here we have fixed an isomorphism between $H^{2}(M, \mathbb{Z})$ with a fixed lattice $L_{K 3}$. It is a nontrivial fact that the period map is a global isomorphism, called the global Torelli theorem for K3 surfaces.

If we restrict to K3 surfaces which are projective, then the moduli space of marked projective K3 surfaces is

$$
S O^{+}(2, k) / S O(2) \times S O(k)
$$

with $1 \leq k \leq 18$ depending on the rank of $H^{2}(M, \mathbb{Z}) \cap H^{1,1}(M)$. This is a Hermitian symmetric space of type IV. Note that every K3 surface can be deformed to a projective surface.

Every Einstein metric $g$ on $M$ is automatically Kähler by observations of Hitchin and Todorov. Therefore it determines a $S^{2}$-family of complex structures on $M$. The analog of the period map for Einstein metrics on $M$ associates to each Einstein metric $g$ the span of $\left[\omega_{I}\right],\left[\omega_{J}\right]$ and $\left[\omega_{K}\right]$ in $H^{2}(M, \mathbb{R})$, which coincides with $H_{+}^{2}(M)$ the space of self-dual harmonic two forms on $M$. Thus the moduli space of marked (orbifold) Einstein metrics on $M$ with unit volume is

$$
S O^{+}(3,19) / S O(3) \times S O(19) .
$$

If we allow the volume of $M$ to vary, then this adds a $\mathbb{R}_{+}$-factor to the above moduli space. From physical motivations, we also consider B-fields which are elements in $H^{2}(M, U(1))$. Then this extended moduli space [2] is isomorphic to

$$
\frac{S O^{+}(3,19)}{S O(3) \times S O(19)} \times \mathbb{R}_{+} \times H^{2}(M, \mathbb{R}) \simeq \frac{S O^{+}(4,20)}{S O(4) \times S O(20)} .
$$

The above isomorphism is defined as follows: The lattice of the total cohomology is

$$
H^{*}(X, \mathbb{Z}) \simeq\left(-E_{8}\right) \oplus\left(-E_{8}\right) \oplus\left(\begin{array}{ll}
0 & 1 \\
1 & 0
\end{array}\right) \oplus\left(\begin{array}{ll}
0 & 1 \\
1 & 0
\end{array}\right) \oplus\left(\begin{array}{ll}
0 & 1 \\
1 & 0
\end{array}\right) \oplus\left(\begin{array}{ll}
0 & 1 \\
1 & 0
\end{array}\right)
$$


and it has signature $(4,20)$. Given any $\left(H_{+}, v, B\right)$ we associate to it a spacelike 4-dimensional space in $H^{*}(M, \mathbb{R})$ spanned by $x-q(x, B)[M]$ with $x \in$ $H_{+}$, together with $1+B+\left(v-\frac{1}{2} q(B)\right)[M]$.

There are conjectural dualities between the geometry, or more precisely the physics, of K3 surfaces and flat tori $T^{d}$ coupled with flat $E_{8} \times E_{8}$-bundles for small $d$. Recall that the moduli space of flat tori is

$$
S O^{+}(d, d) / S O(d) \times S O(d) .
$$

When $d$ equals 2 (resp. 3 and 4), the corresponding structures on K3 surfaces for this duality are algebraic elliptic K3 surfaces with sections (resp. Einstein metrics and Einstein metrics with $B$-fields).

Bundles over K3 surfaces. For any vector bundle $E$ over a K3 surface $M$ with a fixed complex structure $J$, the integrability condition $F_{A}^{0,2}=0$ for a connection $D_{A}$ to define a holomorphic structure on $E$ is equivalent to $F_{A} \wedge \Omega_{J}=0$ where $\Omega_{J}=\omega_{I}-i \omega_{K}$. Note that $F_{A}^{0,2}=F_{A} \wedge \omega_{J}=0$ is identical to the ASD equation $F_{A}^{+}=0$ and this system of equations is equivalent to $F_{A}^{0,2}=0$ with respect to $I, J$ and $K$, i.e. a tri-holomorphic bundle. This is also the same as the following system of equations,

$$
F_{A} \wedge \omega_{I}=F_{A} \wedge \omega_{J}=F_{A} \wedge \omega_{K}=0 .
$$

Recall that $F_{A} \wedge \omega_{J}$ is the moment map for a gauge group action on the space of unitary connections on $E$. Therefore, the moduli space $\mathcal{M}^{A S D}$ of ASD connections, or polystable bundles, on $M$ can be regarded as a hyperkähler quotient $\mathcal{A}(E) / / / \mathcal{G}(E)$. In particular, it is a hyperkähler manifold (section $11)$, but not necessarily compact.

Using the algebraic geometry approach, Mukai [109] showed that the moduli space of polystable coherent sheaves on $M$ admits a canonical holomorphic symplectic form. Examples of such include the Hilbert scheme of $n$-points in $M$ and the universal compactified Jacobians of curves in $M$.

Holomorphic curves vs special Lagrangians in K3. Given any real surface $C$ in $M$, it is a $J$-holomorphic curve if and only if $\left.\Omega_{J}\right|_{C}=0$, i.e. $C$ is a $J$-complex Lagrangian submanifold in $\left(M, \Omega_{J}\right)$. In particular, the normal bundle of $C$ is isomorphic to the cotangent bundle of $C$. For instance, every rational curve in $M$ is a $(-2)$-curve in the sense that the degree of the normal bundle is -2 . Since $\Omega_{J}=\omega_{I}-i \omega_{K}, C$ is a Lagrangian submanifold in $M$ with respect to both the symplectic forms $\omega_{I}$ and $\omega_{K}$. Of course, this is also equivalent to

$$
\left.\omega_{K}\right|_{C}=0 \text { and }\left.\operatorname{Im} \Omega_{K}\right|_{C}=0,
$$

i.e. $C$ is a special Lagrangian submanifold in the Calabi-Yau surface $\left(M, K, \omega_{K}, \Omega_{K}\right)$. Therefore, if $(M, J)$ has an elliptic fibration with section, then the same fibration is a special Lagrangian fibration with section on $\left(M, K, \omega_{K}, \Omega_{K}\right)$.

In an elliptic fibration with section on a K3 surface $M$, the section $S$ is always a smooth rational curve, i.e. $\mathbb{C P}^{1}$. In the generic case, singular fibers 
are nodal rational curves with one node. The total number of singular fibers is 24. This is because the Euler characteristic $e(M)=24$ and smooth elliptic fibers have zero Euler characteristic.

Yau-Zaslow formula. Motivated from physical considerations, YauZaslow [138] found an amazing formula for the number of rational curves on K3 surfaces in terms of a quasi-modular form. This conjectural formula was generalized by Göttsche [49] to arbitrary genus (and arbitrary projective surface): Suppose $C$ is a holomorphic curve in $M$ representing a cohomology class $[C]$ with $q(C)=2 d-2$ and its divisibility, or index, as $r$. If $C$ is a smooth curve, then $d$ is equal to the genus of $C$ and also to the dimension of the linear system of $C$. If we denote the number of genus $g$ curves in $X$ representing $[C]$ as $N_{g}(d, r)$. Then the Gottsche-Yau-Zaslow formula says that

$$
\begin{aligned}
\sum_{d \geq 0} N_{g}(d, r) q^{d} & =\left(\sum_{k=1}^{\infty} k\left(\sum_{d \mid k} d\right) q^{k-1}\right)^{g} \prod_{d \geq 1}\left(\frac{1}{1-q^{d}}\right)^{24} \\
& =\left(\frac{d}{d q} G_{2}(q)\right)^{g} \frac{q}{\Delta(q)} .
\end{aligned}
$$

where $G_{2}(q)$ is the Eisenstein series. Notice that a generic Kähler K3 surface has no curve at all. Using the family Gromov-Witten invariants for the twistor family of complex structures on $M$, Bryan-Leung [19][20] gives a well-defined definition of $N_{g}(d, f)$. When $[C]$ is a primitive class, i.e. $r=1$, this formula was proved in $[\mathbf{1 9}]$ and when $g=0$, i.e. the original $\mathrm{YZ}$ conjecture, it was proved by Klemm-Maulik-Pandharipande-Scheidegger [74].

\section{Calabi-Yau 3-folds}

Calabi-Yau manifolds $M$ of complex dimension 3 have particularly rich geometry. It is related to the fact that $M \times S^{1}$ is a $G_{2}$-manifold and their geometries are governed by the largest normed division algebra, namely the octonion $\mathbb{O}$ (see section 10). Physically this is also the most important dimension in superstring theory.

7.1. Moduli of CY threefolds. When $M$ is a Calabi-Yau threefold with a fixed holomorphic volume form $\Omega$, there are natural cubic forms defined on $H^{1}\left(M, T_{M}^{*}\right)$ and $H^{1}\left(M, T_{M}\right)$, called the $A$-Yukawa coupling $\mathcal{Y}_{A}$ and the $B$-Yukawa coupling $\mathcal{Y}_{B}$ defined as follows: Recall that every element $\phi \in H^{1}\left(M, T_{M}^{*}\right) \cong H^{1,1}(M)$ can be represented by an $(1,1)$-form, then

$$
\begin{gathered}
\mathcal{Y}_{A}: \bigotimes^{3} H^{1}\left(M, T_{M}^{*}\right) \rightarrow \mathbb{C} \\
\mathcal{Y}_{A}\left(\phi_{1}, \phi_{2}, \phi_{3}\right)=\int_{M} \phi_{1} \wedge \phi_{2} \wedge \phi_{3} .
\end{gathered}
$$


If we represent $\eta_{i} \in H^{1}\left(M, T_{M}\right)$ by an element in $\Omega^{0,1}\left(M, T_{M}\right)$, then wedging them together and contracting with $\Omega$ gives $\left.\left(\eta_{1} \wedge \eta_{2} \wedge \eta_{3}\right)\right\lrcorner \Omega \in \Omega^{0,3}(M)$. Then

$$
\begin{gathered}
\mathcal{Y}_{B}: \bigotimes^{3} H^{1}\left(M, T_{M}\right) \rightarrow \mathbb{C} \\
\left.\mathcal{Y}_{B}\left(\eta_{1}, \eta_{2}, \eta_{3}\right)=\int_{M} \Omega \wedge\left(\eta_{1} \wedge \eta_{2} \wedge \eta_{3}\right)\right\lrcorner \Omega .
\end{gathered}
$$

The moduli space $\mathcal{M}_{c p x}$ of complex structures on Calabi-Yau 3-folds is a projective special Kähler manifold, as first observed by Bryant-Griffiths [25] (see also [43]). If we include the choice of a holomorphic volume form on $M$, then this extended moduli space $\widetilde{\mathcal{M}}_{c p x}$ is a $\mathbb{C}^{\times}$-bundle over $\mathcal{M}_{c p x}$ and it is a special Kähler manifold.

Let us recall the definition of special Kähler manifold. For any Kähler manifold, its Kähler form is locally determined by a real valued function $\phi$, called the Kähler potential, i.e. $\omega=i \bar{\partial} \partial \phi(z, \bar{z})$, while for special Kähler manifold, it is determined by a holomorphic function $\mathcal{F}(z)$, called the prepotential, which satisfies

$$
\phi=\frac{1}{2} \operatorname{Im}\left(\frac{\partial \mathcal{F}}{\partial z^{i}} \bar{z}^{i}\right)
$$

for suitable holomorphic coordinates $z$ 's. From an intrinsic point of view, a Kähler manifold $(\mathcal{M}, \omega)$ with a torsion free flat symplectic connection $\nabla$ is a special Kähler manifold if $\nabla \wedge J=0$. Such a manifold has a local holomorphic function $\mathcal{F}$, called the prepotential, which governs the special Kähler geometry. In terms of a suitable dual holomorphic Darboux coordinates $z$ 's and $w$ 's, we have

$$
w_{j}=\frac{\partial \mathcal{F}}{\partial z^{j}},
$$

for all $j$. When $X$ is a special Kähler manifold, its cotangent bundle $M=$ $T^{*} X$ admits a natural hyperkähler structure.

To define this structure on $\widetilde{\mathcal{M}}_{c p x}$, we note that the tangent space at $[M] \in$ $\mathcal{M}_{c p x}$ (resp. $\left.[(M, \Omega)] \in \widetilde{\mathcal{M}}_{c p x}\right)$ is naturally identified with $H^{1}\left(M, T_{M}\right) \cong$ $H^{2,1}(M)$ (resp. $\left.H^{3,0}(M) \oplus H^{2,1}(M)\right)$. Notice that the Poincaré pairing on the complex vector space $H^{3}(M, \mathbb{C})$ defines a holomorphic symplectic form and such that $H^{3,0}(M) \oplus H^{2,1}(M)$ is a complex Lagrangian subspace in it. We choose a symplectic basis $A^{i}$ 's, $B_{i}$ 's for the middle homology group $H_{3}(M, \mathbb{Z})$ modulo torsion, i.e.

$$
A^{i} \cap A^{j}=0=B_{i} \cap B_{j} \quad \text { and } \quad A^{i} \cap B_{j}=\delta_{j}^{i} .
$$

Then

$$
z^{i}=\int_{A^{i}} \Omega
$$


defines a local holomorphic coordinate system on $\widetilde{\mathcal{M}}_{c p x}$. Similarly

$$
w_{i}=\int_{B_{i}} \Omega
$$

define another such coordinate and it is related to $z^{i}$ 's by a holomorphic Legendre transformation, i.e. there exists a local holomorphic function $\mathcal{F}\left(z^{i}\right)$ such that

$$
w_{i}=\frac{\partial \mathcal{F}}{\partial z^{i}}
$$

This gives the prepotential function on $\widetilde{\mathcal{M}}_{c p x}$.

In terms of this coordinate $z^{i}$, s on $\widetilde{\mathcal{M}}_{c p x}$, one has

$$
\mathcal{Y}_{B}=\nabla^{3} \mathcal{F} \text {. }
$$

Such structures determine a so-called Frobenius manifold structure on $\widetilde{\mathcal{M}}_{c p x}$.

In general, the cotangent bundle of any special Kähler manifold admits a canonical hyperkähler structure. In our situation, the fibers of the cotangent bundle of $\widetilde{\mathcal{M}}_{c p x}$ can be identified as the universal covering of the intermediate Jacobian $\mathcal{J}(M)$,

$$
\mathcal{J}(M)=H^{3}(M, \mathbb{Z}) \backslash H^{3}(M, \mathbb{C}) /\left(H^{3,0}(M) \oplus H^{2,1}(M)\right) .
$$

Therefore $H^{3}(M, \mathbb{Z}) \backslash T^{*} \widetilde{\mathcal{M}}_{c p x}$ is the universal intermediate Jacobian $\mathcal{J}^{\text {univ }}$ for Calabi-Yau threefolds. It admits a hyperkähler structure with a complex Lagrangian fibration over the extended moduli space of Calabi-Yau threefolds,

$$
\pi: \mathcal{J}^{\text {univ }} \rightarrow \widetilde{\mathcal{M}}_{c p x}
$$

We remark that the second fundamental form of a Lagrangian submanifold $L$ is always a symmetric cubic tensor in $\Gamma\left(L, S y m^{3} T_{L}^{*}\right)$. For a Lagrangian fibration, if we integrate the second fundamental forms for fibers, then we obtain a cubic tensor on the base of the fibration. For our complex Lagrangian fibration $\mathcal{J}^{\text {univ }} \rightarrow \widetilde{\mathcal{M}}_{c p x}$, this cubic tensor is nothing but the B-Yukawa coupling $\mathcal{Y}_{B}$.

7.2. Curves and surfaces in Calabi-Yau threefolds. A real codimension two submanifold $S$ in $M$ is a complex surface if and only if $\left.\Omega\right|_{S}=0$. Given any complex surface $S$ in $M$, its normal bundle $N_{S / M}$ equals to the canonical line bundle $K_{S}$ of $S$. When $S$ is a del Pezzo surface, for instance $\mathbb{C P}^{2}, N_{S / M}$ is negative and therefore $S$ can be contracted to a point. We call $K_{S}$ a local Calabi-Yau manifold. When $S$ is a Calabi-Yau surface, i.e. a K3 surface or an Abelian surface, then $N_{S / M}$ is trivial and $S$ can be deformed and gives a complex surface fibration structure on $M$. Similarly when $C$ is an elliptic curve, it often happens that $C$ is a fiber of an elliptic fibration on $M$. There has been much studies on elliptic Calabi-Yau threefolds, including the geometry of its Kähler cone by Wilson, stable bundles over them by Donagi, Friedman, Morgan and others. 
Flops, smoothing and extremal transitions. When $C$ is a smooth rational curve in $M$, then

$$
N_{C / M} \cong O(-d) \oplus O(d-2)
$$

for some integer $d$. In the generic situation with $N_{C / M} \cong O(-1) \oplus O(-1)$ negative, $C$ is called an $(-1,-1)$-curve, then $C$ can be contracted to a rational double point, i.e. locally given by

$$
\left\{z_{1}^{2}+z_{2}^{2}+z_{3}^{2}+z_{4}^{2}=0\right\} \subset \mathbb{C}^{4} .
$$

There is another small resolution of this rational double point and gives another $(-1,-1)$-curve $C^{\prime}$ as the exceptional set. This birational transformation is called a simple flop. Suppose $M^{\prime}$ is obtained by flopping $C$ in $M$, the cohomology rings of $M$ and $M^{\prime}$ are different, in particular the topology of $M$ changes under flops. Nevertheless, if we consider the quantum product by including contributions from genus zero GW invariants to the classical cup product, then the quantum cohomology ring of $M$ and $M^{\prime}$ are the same [86]. In general, we expect that the quantum geometries, or the string theories, of $M$ and $M^{\prime}$ are the same.

We could also consider the smoothing of the rational double point by deforming the complex structure to

$$
\left\{z_{1}^{2}+z_{2}^{2}+z_{3}^{2}+z_{4}^{2}=t\right\} \subset \mathbb{C}^{4},
$$

for small non-zero $t$. Topologically, this process replaces the singular point by a (Lagrangian) $S^{3}$, which is given by $\left\{z_{1}^{2}+z_{2}^{2}+z_{3}^{2}+z_{4}^{2}=t\right\} \cap \mathbb{R}^{4}$ when $t>0$. This is called a vanishing cycle for the isolated singular point. Indeed this is the mirror to the blowing up process which replaces the singular point by a (holomorphic) $S^{2} \cong \mathbb{C P}^{1}$. The process of contracting a $(-1,-1)$ curve followed by a smoothing is called an extremal transition. The mirror symmetry conjecture has predicted several surprising consequences for such transitions, including a formula relating open GW invariants on $O(-1) \oplus$ $O(-1)$ over $S^{2}$ with the Chern-Simons theory on $S^{3}$.

Thus the rational double point can be resolved in three different ways. One by smoothing (with a $S^{3}$ vanishing cycle) and two by blowing up (with a $S^{2}$ exceptional cycle). When we view these from the $G_{2}$ perspectives, all three look the same and they are related by a triality symmetry (section 8.5).

$G W$-invariants and multiple cover formula for $C Y^{3}$. For any Calabi-Yau 3 -fold $M$, the moduli space of genus $g$ holomorphic curves $C$ in $M$ always has expected dimension zero. Thus we denote the $\mathrm{GW}$-invariant which counts the number of genus $g$ curves in $M$ representing a class $\beta \in H_{2}(M, \mathbb{Z})$ as $N_{g}^{\beta}(M)$. Notice that GW-invariants count the number of stable maps from genus $g$ curves to $M$ and every curve $C \subset M$ representing $\beta \in H_{2}(M, \mathbb{Z})$ will contribute to the GW-invariant for the class $d \beta$ because of a degree $d$ stable map $f: \Sigma \rightarrow M$ could multiply cover its image $f(\Sigma)=C$. Such contributions to the GW-invariants should be subtracted in order to count the number of 
curves in $M$. The multiple cover formulas tell us how each curve in the class $\beta$ contributes to the $\mathrm{GW}$-invariant for the class $d \beta$. In the genus zero case, the contribution is $d^{-3}$ when $C$ is an $(-1,-1)$-curve. There are also multiple cover formula for other contractible curves $[\mathbf{1 7}]$.

Superpotential for curves. Dimension being three plays a special role here as holomorphic curves are critical points of the following Chern-Simons type functional $\Phi$ among real surfaces $\Sigma$ in $M$,

$$
\Phi(\Sigma)=\int_{B} \Omega
$$

where $\Omega \in \Omega^{3,0}(M)$ is the holomorphic volume form on $M$ and $B$ is a real three dimensional singular chain in $M$ with $\partial B=\Sigma-\Sigma_{0}$ for any fixed background surface $\Sigma_{0}$ in $M$.

Thus the universal moduli space $\widetilde{\mathcal{M}}_{\text {curve }}^{\text {univ }}$ of curves in Calabi-Yau 3-folds is expected to be a generic finite cover of $\widetilde{\mathcal{M}}_{c p x}$. We recall the universal Abel-Jacobi map

$$
A J: \widetilde{\mathcal{M}}_{\text {curve }}^{\text {univ }} \rightarrow \mathcal{J}^{\text {univ }} .
$$

To explain it, we fix a background $C_{0}$ and for any holomorphic curve $C$ in $M$ homologous to $C_{0}$ we define $A J(C) \in H^{3}(M, \mathbb{Z}) \backslash H^{3}(M, \mathbb{C}) /\left(H^{3,0}(M)\right.$ $\left.\oplus H^{2,1}(M)\right)$ by evaluating it on any cohomology class $\eta \in H^{3}(M, C)$ as follows,

$$
A J(C)[\eta]=\int_{B} \eta
$$

where $B$ is any singular chain in $M$ with $\partial B=C-C_{0}$. The map $A J$ defines a complex Lagrangian subspace in $\mathcal{J}^{\text {univ }}[\mathbf{1 2 1}]$. Locally, it is given by the graph of an exact holomorphic one form $d \Phi$ on $\widetilde{\mathcal{M}}_{c p x}$, where

$$
\begin{gathered}
\Phi: \widetilde{\mathcal{M}}_{\text {curve }}^{\text {univ }} \rightarrow \mathbb{C} \\
\Phi(M, C)=\int_{B} \Omega .
\end{gathered}
$$

This is called the superpotential in the physics literature.

Gopakumar-Vafa conjecture. A holomorphic curve $C \subset M$ coupled with a holomorphic line bundle $L$ over $C$ is called a $B P S$ state in string theory. The moduli space $\mathcal{M}_{B P S}(M)$ of such pairs $(C, L)$ carries a forgetful map to the moduli space of curve in $M$,

$$
\pi: \mathcal{M}_{B P S}(M) \rightarrow \mathcal{M}_{\text {curve }}(M) .
$$

One would like to find a good compactification of $\mathcal{M}_{B P S}(M)$. For K3 surfaces, Yau and Zaslow [138] studied this space and derived an amazing formula for the generating functions of the number of rational curves in K3 surfaces, the Yau-Zaslow formula. From physical considerations, Gopakumar and Vafa [48] conjectured that the cohomology group $H^{*}\left(\overline{\mathcal{M}}_{B P S}(M)\right)$ should admits an $s l(2, \mathbb{R}) \times s l(2, \mathbb{R})$-action and suitable combinations of 
its multiplicities are called the BPS number denoted $n_{g}^{\beta}(M) \in \mathbb{Z}$ and they determine Gromov-Witten invariants of $M$ of every genus explicitly by the following formula,

$$
\sum_{\substack{g \geq 0 \\ \beta \in H_{2}(M, \mathbb{Z})}} N_{g}^{\beta}(M) u^{2 g-2} q^{\beta}=\sum_{\substack{g \geq 0 \\ \beta \in H_{2}(M, \mathbb{Z})}} n_{g}^{\beta}(M) \sum_{k>0} \frac{1}{k}\left(2 \sin \frac{k u}{2}\right)^{2 g-2} q^{k \beta}
$$

where $N_{g}^{\beta}(M)$ is the GW-invariant and $n_{g}^{\beta}(M)$ is the BPS number.

7.3. Donaldson-Thomas bundles over Calabi-Yau threefolds. Holomorphic bundles $E$ over a Calabi-Yau threefold $M$ is another type of BPS states in string theory. Recall that a connection $D_{A}$ defines a holomorphic structure on $E$ if and only if $\left(F_{A}\right)^{0,2}=0$. Similar to the special feature of flatness $F_{A}=0$ on three manifolds, $\left(F_{A}\right)^{0,2}=0$ is the Euler-Lagrange equation for the holomorphic Chern-Simons functional $C S_{h o l}$ on Calabi-Yau threefolds $M$,

$$
C S_{h o l}\left(D_{A}\right)=\int_{M} \Omega \wedge \operatorname{Tr}\left(A \wedge d A+\frac{2}{3} A \wedge A \wedge A\right),
$$

where $D_{A}=D_{A_{0}}+A$ for some fixed background connection $D_{A_{0}}$. Equivalently,

$$
C S_{h o l}\left(D_{A}\right)=\int_{M \times[0,1]} \Omega \wedge \operatorname{Tr} F_{\bar{A}}^{2}
$$

with $D_{\bar{A}}$ a connection over $M \times[0,1]$ extending $D_{A_{0}}$ and $D_{A}$ on its boundaries. This is similar to the functional $\Phi$ for real surfaces in $M$. We can therefore have an analog of the Abel-Jacobi map $A J_{b d l}: \widetilde{\mathcal{M}}_{b d l}^{\text {univ }} \rightarrow \mathcal{J}^{\text {univ }}$ on the universal moduli space $\widetilde{\mathcal{M}}_{b d l}^{\text {univ }}$ of holomorphic bundle over Calabi-Yau 3 -folds and it is an Lagrangian with superpotential given by

$$
\begin{gathered}
\Phi_{b d l}: \widetilde{\mathcal{M}}_{b d l}^{\text {univ }} \rightarrow \mathbb{C} \\
\Phi\left(M, D_{A}\right)=\int_{M \times[0,1]} \Omega \wedge \operatorname{Tr} F_{\bar{A}}^{2} .
\end{gathered}
$$

Indeed, this is a special case of the special geometry of cycles in $G_{2}$-manifolds [73] (section 8).

For Calabi-Yau threefolds $M$, using techniques from algebraic geometry, Donaldson and Thomas [40] define a count $D T_{M}$ for the number of stable holomorphic bundles over $M$ which is invariant under deformations of complex structures on $M$. Indeed one can allow $E$ to be singular as well, namely a coherent sheaf on $M$. For example every holomorphic curve $C$ in $M$ defines an ideal sheaf $\mathcal{I}_{C}$ on $M$ which is automatically stable and the corresponding 
Donaldson-Thomas invariants $D T_{M}$ are conjectured by Maulik-NekrasovOkounkov-Pandharipande [107] to be related to GW-invariants by

$$
\frac{Z_{D T, \beta}(q)}{Z_{D T, 0}(q)}=Z_{G W, \beta}(u)
$$

after a change of coordinate $e^{i u}+q=0$. Here $Z_{D T, \beta}(q)=\sum_{n} I_{n, \beta} q^{n}$ and $Z_{G W, \beta}(u)=\sum_{g} N_{g, \beta} u^{2 g-2}$. It is proven that $Z_{D T, 0}(q)$ is given by the MacMahon function $M(q)=\prod_{n \geq 1}\left(1-q^{n}\right)^{-n}$.

Recently Pandharipande and Thomas [113] defined a related invariant by counting stable pairs $O_{M} \rightarrow O_{C}$ in the derived category $D^{b}(M)$ instead of $\mathcal{I}_{C}$ and this has the effect of getting rid of the contributions of embedded points in $C$ to the DT-invariants. They are called the PT-invariants and denote $Z_{P T, \beta}(u)$. They conjectured the following relationship

$$
\frac{Z_{D T, \beta}(q)}{Z_{D T, 0}(q)}=Z_{P T, \beta}(u)
$$

Pandharipande and Thomas formally defined BPS count $n_{g}^{\beta}$ in terms of PT-invariants for irreducible curve classes.

7.4. Special Lagrangian submanifolds in $\mathbf{C Y}^{3}$. An A-brane $\left(L, D_{A}\right)$ in a Calabi-Yau manifold $M$ consists of a Lagrangian submanifold $L$ in $M$ together with a flat $U(1)$-connection over $L$. In complex dimension three, A-branes are critical points of the following Chern-Simons type functional

$$
\int_{L_{0}}^{L_{1}}\left(\omega+F_{A}\right)^{2}
$$

where $\left(L_{t}, D_{A_{t}}\right)$ is a smooth path of A-branes connecting a background $\left(L_{0}, D_{A_{0}}\right)$ to $\left(L_{1}, D_{A_{1}}\right)=\left(L, D_{A}\right)$.

The moduli space of A-branes has a natural symplectic structure and admits a Hamiltonian action by the group of gauge transformation $\mathcal{G}=$ $C^{\infty}\left(M, S^{1}\right)$ of the flat line bundle over $L$. The corresponding moment map $\mu$ is given by

$$
\mu\left(L, D_{A}\right)=\left.\operatorname{Im} \Omega\right|_{L}
$$

Therefore the symplectic quotient is the moduli space of special Lagrangian submanifolds in $M[\mathbf{1 1 9}]$.

Because of these special properties in dimension three, one might expect to be able to count the number of special Lagrangian spheres in Calabi-Yau threefolds, as studied by Joyce [72] and other people.

7.5. Mirror symmetry for Calabi-Yau threefolds. General mirror symmetry conjectures are expected to hold true for Calabi-Yau manifolds of any dimension. The conjecture was originated from the studies of string theory in which the Calabi-Yau manifolds always have complex dimension 
three. Indeed there are many mysterious dualities for the geometry/physics of Calabi-Yau threefolds, which are not shared by Calabi-Yau manifolds of higher dimensions. A reason that Calabi-Yau threefold geometry is much richer is due to the fact that $S U(3) \subset G_{2}=A u t(\mathbb{O})$. As a matter of fact, many of these dualities comes from dualities/triality in $G_{2}$-geometry/physics conjecturally.

We have explain that the complex geometry and the symplectic geometry for Calabi-Yau threefolds are expected to be unchanged under flops and (mirror) transformed to each other under extremal transitions. This includes the large $N$ duality for Chern-Simons theory of knots in $S^{3}$. Nekrasov argued physically that the large $N$ Chern-Simons theory on $S^{3}$ can also be described in terms of the geometry of the moduli spaces of ASD connections on $\mathbb{R}^{4}$. Very roughly speaking, the reason is as $S^{3}$ shrinks to a point in its cotangent bundle and creates a conical singularity, the physics of the ten dimensional spacetime $T^{*} S^{3} \times \mathbb{R}^{4}$ can be approximated by the physics of the base manifold $\mathbb{R}^{4}$.

Atiyah-Witten [7] observed that both the $O(-1,-1)$ bundle over $S^{2}$ where ordinary flops take place and the cotangent bundle over $S^{3}$ where extremal transitions take place are quotients of the spinor bundle over $S^{3}$, a seven dimensional $G_{2}$-manifold, by $S^{1}$ in different ways. These different ways are connected by $G_{2}$ triality flops. Thus when lifting to the $G_{2}$-geometry, flops and extremal transitions are the same operation. Only when we quotient it by $S^{1}$ to obtain Calabi-Yau manifolds, then we break the symmetry. We expect that $G_{2}$-flops on $S^{3}$ will preserve both the associative geometry and the coassociative geometry on $G_{2}$-manifolds.

The closed string theory on $M \times \mathbb{R}^{3,1}$ is conjecturally dual to the Mtheory on $\left(S^{1} \times M\right) \times \mathbb{R}^{3,1}$. This led to the conjecture of Gopakumar-Vafa [48] and eventually the many developments of counting curves in Calabi-Yau threefolds, including the Donaldson-Thomas invariants, the PandharipandeThomas invariants and their relationships with the Gopakumar-Vafa BPSinvariants.

For the open string theory on $X \times \mathbb{R}^{3,1}$ with the boundaries of string lying on a special Lagrangian submanifold $L$ in $M$, the conjectural M-theory dual is a $G_{2}$-manifold $X$ which admits a $S^{1}$-fibration to $M$ whose fibers over $L$ degenerate to points. For M-theory on more general $G_{2}$-manifolds $X$, the conjectural duals are Calabi-Yau threefolds $M$ coupled with $E_{8} \times E_{8}$-bundles, as studied by Gukov-Yau-Zaslow [58].

Another link between Calabi-Yau threefolds and $G_{2}$-manifolds comes from the topological M-theory [33] in which the Hitchin volume functional on the space of three forms is being quantized. The natural projectively flat connection over the parameter space can be interpreted as the BCOV anomaly equation [12] for Calabi-Yau threefolds. The BCOV theory was originally arisen from studies of the Kodaira-Spencer theory of gravity and their mirror symmetry between A- and B-models. The BCOV mirror conjecture is a powerful tool to compute higher genus Gromov-Witten invariants 
for Calabi-Yau threefolds as their generating function is the partition function in A-model of this theory.

\section{8. $G_{2}$-geometry}

8.1. $G_{2}$-manifolds. A seven dimensional Riemannian manifold $(M, g)$ is called a $G_{2}$-manifold if it has a parallel vector cross product (VCP) on its tangent spaces.

Recall that a linear homomorphism $J: \mathbb{R}^{m} \rightarrow \mathbb{R}^{m}$ is an orthogonal complex structure if and only if it satisfies (i) $J u \perp u$ and (ii) $|J u|=|u|$ for any vector $u$. The only constraint is $m$ being an even integer. Now we generalize it to a bilinear homomorphism $\times: \mathbb{R}^{m} \otimes \mathbb{R}^{m} \rightarrow \mathbb{R}^{m}$ by requiring (i) $u \times v$ perpendicular to both $u$ and $v$ and (ii) $|u \times v|$ equals the area of the parallelogram spanned by $u$ and $v$. Obviously the standard vector product, or sometimes called the cross product, in $\mathbb{R}^{3}$ is an example of such. One way to express this product is by identifying $\mathbb{R}^{3}$ with $\operatorname{Im} \mathbb{H}$ and defining $u \times v=\operatorname{Im}(u \cdot v)$ with $u \cdot v$ the product of quaternions. The same formula also defines a VCP on the imaginary octonions $\mathbb{R}^{7} \cong \operatorname{Im} \mathbb{O}$. In fact these are all VCPs [50].

The octonion product can also be recovered from the VCP, and thus the group of isometries of $\mathbb{R}^{7}$ preserving $\times$ equals the group of algebra automorphisms of $\mathbb{O}$, which is the exceptional Lie group $G_{2}$. Therefore $G_{2^{-}}$ manifolds do have holonomy groups $G_{2}$. Since $S U(3) \subseteq G_{2}$, the product of any Calabi-Yau threefold with $\mathbb{R}$ is always a $G_{2}$-manifold. Like CY manifolds, $G_{2}$-manifold always Einstein manifolds, i.e. $R c=0$.

The analog of the Kähler form $\omega(u, v)=g(J u, v)$ is the $G_{2}$-form $\Omega \in$ $\Omega^{3}(M)$ given by

$$
\Omega(u, v, w)=g(u \times v, w) .
$$

When $M=X \times \mathbb{R}$ with $X$ being a Calabi-Yau threefold with Kähler form $\omega_{X}$ and holomorphic volume form $\Omega_{X}$, then we have

$$
\Omega=\operatorname{Re} \Omega_{X}-\omega_{X} \wedge d t
$$

$\Omega$ is a three form analog of a symplectic form as for any $x \in M, \Omega_{x} \in \Lambda^{3} T_{x}^{*} M$ lies in an open (positive) $G L(7, \mathbb{R})$-orbit. Unlike a symplectic form, the metric $g$ is determined by $\Omega$,

$$
g(u, v) v_{M}=\iota_{u} \Omega \wedge \iota_{v} \Omega \wedge \Omega .
$$

Furthermore, $\nabla \Omega=0$ can be reduced to $\Delta \Omega=0$. Thus a $G_{2}$-manifold can be characterized as a seven dimensional manifold processing a harmonic positive three form $\Omega$.

Despite such simplifications, there is no general existence result for compact $G_{2}$-manifolds, analogous to Yau's theorem for Calabi-Yau manifolds. Using singular perturbation method to resolve singularities of $T^{7} / \Gamma$ for certain finite group $\Gamma$-action, Joyce [70] constructed the first nontrivial compact examples. Explicit noncompact examples were constructed earlier by Bryant, 
Salamon and others by imposing symmetries to reduce the $G_{2}$-condition to an ODE. This included total spaces of the spinor bundle $S\left(S^{3}\right)$ over $S^{3}$, the self-dual two-form bundles $\Lambda_{+}^{2}\left(S^{4}\right)$ and $\Lambda_{+}^{2}\left(\mathbb{C P}^{2}\right)$.

Analogous to the $(p, q)$-decomposition for Kähler manifolds, in the $G_{2}$ setting, we have

$$
\begin{aligned}
& \Omega^{1}(M)=\Omega_{7}^{1} \\
& \Omega^{2}(M)=\Omega_{7}^{2} \oplus \Omega_{14}^{2} \\
& \Omega^{3}(M)=\Omega_{1}^{3} \oplus \Omega_{7}^{3} \oplus \Omega_{27}^{3},
\end{aligned}
$$

and $\Omega_{d}^{k} \cong \Omega_{d}^{7-k}$ by the Hodge star operator $*$. These components correspond to the irreducible decomposition of $G_{2}$ acting on $\Lambda^{*} \mathbb{R}^{7}$. Here the subscript indicate the dimension of these irreducible components inside $\Lambda^{*} \mathbb{R}^{7}$. We have (i) $\Lambda_{1}^{3}$ is spanned by $\Omega$, (ii) $\Lambda_{7}^{1} \cong \Lambda_{7}^{2} \cong \Lambda_{7}^{3}$ via $T^{*} \stackrel{g}{\rightarrow} T \stackrel{\lrcorner \Omega}{\rightarrow} \Lambda^{2} T^{*}$ and $T^{*} \stackrel{\wedge \Omega}{\rightarrow}$ $\Lambda^{4} T^{*} \stackrel{*}{\rightarrow} \Lambda^{3} T^{*}$, (iii) $\Lambda_{14}^{2}$ corresponds to $\mathbf{g}_{2} \subset$ so $(7) \cong \Lambda^{2} \mathbb{R}^{7}$ and (iv) $\Lambda_{27}^{3} \cong$ $\operatorname{Sym}_{0}^{2} \mathbb{R}^{7}$ corresponds to deformations of the $G_{2}$-metrics with fixed volume.

8.2. Moduli of $G_{2}$-manifolds. Given any $G_{2}$-manifold $M$ with $G_{2^{-}}$ form $\Omega$, we define a $G_{2^{-}}$Yukawa coupling as follows

$$
\begin{gathered}
\mathcal{Y}_{\Omega}: \bigotimes^{3} H^{3}(M) \rightarrow \mathbb{R} \\
\left.\mathcal{Y}_{\Omega}\left(\phi_{1}, \phi_{2}, \phi_{3}\right)=\int * \Omega \wedge\left(\tilde{\phi}_{1} \wedge \tilde{\phi}_{2} \wedge \tilde{\phi}_{3}\right)\right\lrcorner \Omega
\end{gathered}
$$

where for any $\phi_{i} \in \Omega^{3}(M), \tilde{\phi}_{i} \in \Omega^{1}\left(M, T_{M}\right)$ is defined by

$$
\iota_{v_{1} \wedge v_{2}} \phi_{i}=\iota_{v_{1} \wedge v_{2} \wedge \tilde{\phi}_{i}} \Omega \in \Omega^{1}(M)
$$

for any vector $v_{1}$ and $v_{2}$. It satisfies

$$
\mathcal{Y}_{\Omega}(\Omega, \Omega, \Omega)=\mathcal{Y}_{\Omega}(\Omega)=\int|\Omega|^{2}=\int \Omega \wedge * \Omega \text {. }
$$

When $M=X \times S^{1}$, then $\mathcal{Y}_{\Omega}$ is a combination of $\mathcal{Y}_{A}$ and $\mathcal{Y}_{B}$ for the CalabiYau threefold $X$.

Unless a compact $G_{2}$-manifold is a metric product $M=X \times S^{1}$ up to a finite cover, the holonomy group is the full $G_{2}$. Via Bochner argument, we have $H^{1}(M)=0$ and therefore $H_{7}^{k}(M)=0$ for any $k$. Thus $H^{3}(M) \cong$ $H_{1}^{3}(M) \oplus H_{27}^{3}(M)$ parametrizes infinitesimal deformations of $G_{2}$-metrics on $M$.

If we fix a marking, i.e. an isomorphism from $H_{3}(M, \mathbb{Z}) /$ Tor to a fixed lattice, then the moduli space $\tilde{\mathcal{M}}_{G_{2}}$ of marked $G_{2}$-structure on $M$ is locally isomorphic to $H^{3}(M)$ and given by

$$
\begin{aligned}
p: \tilde{\mathcal{M}}_{G_{2}} & \rightarrow H^{3}(M) \\
(M, \Omega) & \rightarrow[\Omega] .
\end{aligned}
$$


If we restrict our attention to $\mathcal{M}_{G_{2}} \subset \tilde{\mathcal{M}}_{G_{2}}$ consisting of those $G_{2}$-metrics with unit total volume, then up to a positive constant, the WeilPetersson metric is equal to $\mathcal{Y}_{\Omega}(\Omega,-,-)$, or $\nabla^{2} \mathcal{Y}_{\Omega}(\Omega)$. In particular, the metric is of Hessian type. This is the real analog to the special Kähler structure on the moduli space of complex structures on Calabi-Yau threefolds. Such a geometry is captured by the cubic form $\nabla^{3} \mathcal{Y}_{\Omega}(\Omega)$, which is nothing but the $G_{2}$-Yukawa coupling $\mathcal{Y}_{\Omega}$ itself.

Similarly to the universal intermediate Jacobian in the Calabi-Yau threefolds situation, we construct the bundle

$$
\mathcal{J}_{G_{2}} \rightarrow \tilde{\mathcal{M}}_{G_{2}}
$$

whose fiber over $(M, \Omega)$ is $H^{3}\left(M, S^{1}\right)[\mathbf{7 3}] . \mathcal{J}_{G_{2}}$ has a natural Kähler structure in such a way that the above map is a Lagrangian fibration.

\section{3. (Co-)associative geometry.}

(Co-)associative submanifolds. The analog of the complex geometry defined by $J$ (resp. symplectic geometry defined by $\omega$ ) is the associative geometry defined by $\times$ (resp. coassociative geometry defined by $\Omega$ ) on the $G_{2}$-manifold $M[84]$.

Since $\mathbb{R}^{m}$ admits a VCP only when $m=3$ or $7, \times$-invariant submanifolds in $M$ must have dimension three and they are called associative submanifolds, or instantons. They are calibrated by $\Omega$ and therefore absolute minimal submanifolds in $M$. Infinitesimal deformations of associative submanifolds are parametrized by twisted harmonic spinors and their deformations could be obstructed.

The $G_{2}$-analog of Lagrangian submanifolds are coassociative submanifolds in $M$, which are four dimensional submanifolds $C$ with $\left.\Omega\right|_{C}=0$. They are calibrated by $* \Omega$, like special Lagrangian submanifolds. The normal bundle $N_{C / M}$ is isomorphic to the bundle $\Lambda_{+}^{2}(C)$ of self-dual two forms on $C$. Infinitesimal deformations of $C$ as coassociative submanifolds are parametrized by harmonic self-dual two forms $H_{+}^{2}(C)$ and their deformations are always unobstructed. In particular, their moduli space $\mathcal{M}^{\text {coass }}(M)$ is smooth. Coassociative submanifolds are natural boundary conditions for the free boundary value problem of associative submanifolds with nonempty boundaries.

In the next section, we will explain that the theory of associative submanifolds and coassociative submanifolds in $G_{2}$-manifolds are special examples of instantons and branes in manifolds with VCP. We could also view a $G_{2}$-manifold $M$, or more precisely $M \times S^{1}$, as an $\mathbb{O}$-oriented $\mathbb{O}$-manifold (see section 10). From this point of view, associative (resp. coassociative) submanifolds are $\mathbb{H}$-Lagrangian submanifolds of type I (resp. type II) in $M$.

When $M=X \times S^{1}$, a submanifold of the form $\Sigma \times S^{1}$ (resp. $\left.L \times\{p\}\right)$ is an associative submanifold in $M$ if and only if $\Sigma$ is a holomorphic curve (resp. $L$ is a special Lagrangian with phase zero) in the Calabi-Yau threefold $X$. Similarly, a submanifold of the form $L \times S^{1}$ (resp. $C \times\{p\}$ ) is a coassociative 
submanifold in $M$ if and only if $L$ is a special Lagrangian with phase $\pi / 2$ (resp. $C$ is a complex surface) in $X$.

Analogous to the roles that holomorphic disks play in the intersection theory of Lagrangian submanifolds in symplectic manifolds, one should consider associative submanifolds bounding the intersections of coassociative submanifolds in the $G_{2}$-setting. This theory is also closely related to the Seiberg-Witten theory of the coassociative submanifold $C[\mathbf{9 9}]$.

$G_{2}-T F T$. It is natural to couple (co-)associative submanifolds $C$ with $U(r)$-connections on $E$ over $C$. We consider the following Chern-Simons type functional

$$
\begin{gathered}
C S_{G_{2}}: \operatorname{Map}(C, M) \times \mathcal{A}(C) \rightarrow \mathbb{R} \\
C S_{G_{2}}\left(f_{1}, D_{A_{1}}\right)=\int_{[0,1] \times C} \operatorname{Tr} \exp \left(f^{*}(\Omega+* \Omega)+F_{A}\right),
\end{gathered}
$$

where $f:[0,1] \times C \rightarrow M$ with $f(t, x)=f_{t}(x)$ is an one parameter family of maps joining $f_{1}$ with a background map $f_{0}$. This map is invariant under the natural action of the connected component of the large gauge group $\tilde{\mathcal{G}}$ consisting of $\tilde{g}: E \rightarrow E$

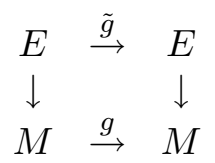

covering a diffeomorphism $g$ of $M$ and linear isomorphisms along fibers. It fits into the following fiber bundle with fiber the usual group of gauge transformations,

$$
\mathcal{G} \rightarrow \tilde{\mathcal{G}} \rightarrow \operatorname{Diff}(C)
$$

When $\operatorname{dim} C=3$,

$$
C S_{G_{2}}\left(f_{1}, D_{A_{1}}\right)=\int_{[0,1] \times C} * \Omega+\frac{1}{2} \operatorname{Tr} F_{A}^{2} .
$$

Critical points are $\left(f_{1}, D_{A_{1}}\right)$ 's with $f_{1}(C)$ as associative submanifold in $M$ and $D_{A_{1}}$ a flat connection over $f_{1}(C)$. Thus the moduli space of critical points,

$$
\left\{d C S_{G_{2}}=0\right\} / \tilde{\mathcal{G}}=\mathcal{M}^{A-c y c l e}(M)
$$

is the moduli space of associative submanifolds coupled with flat bundles, called associative cycles, or simply A-cycles in $M$.

When $\operatorname{dim} C=4$,

$$
C S_{G_{2}}\left(f_{1}, D_{A_{1}}\right)=\int_{[0,1] \times C} \operatorname{Tr} \Omega \wedge F_{A} .
$$

Suppose that $D_{A_{1}}$ is a $U(1)$-connection, then the Euler-Lagrange equations is

$$
\left.\Omega\right|_{f_{1}(C)}=0 \quad \text { and } \quad F_{A_{1}}^{+}=0 .
$$


Thus the moduli space of critical points,

$$
\left\{d C S_{G_{2}}=0\right\} / \tilde{\mathcal{G}}=\mathcal{M}^{C-\text { cycle }}(M)
$$

is the moduli space of coassociative submanifolds coupled with ASD bundles, called coassociative cycles, or simply $C$-cycles in $M$.

Formally, the Witten-Morse theory for $C S_{G_{2}}$ defines topological field theories, called $A$-TFT and $C$-TFT for $G_{2}$-manifolds [91][39].

Moduli of $A$ - and $C$-cycles. The moduli spaces $\mathcal{M}^{A-c y c l e}(M)$ and $\mathcal{M}^{C-\text { cycle }}(M)$ process natural three forms, four forms and cubic tensors, analogous to the $G_{2}$-forms and Yukawa couplings. When $r=1$, the forgetting maps to the moduli spaces of associative and coassociative submanifolds,

$$
\begin{aligned}
& \mathcal{M}^{\text {A-cycle }}(M) \rightarrow \mathcal{M}^{\text {assoc }}(M) \text { and } \\
& \mathcal{M}^{C-\text { cycle }}(M) \rightarrow \mathcal{M}^{\text {coass }}(M)
\end{aligned}
$$

behave somewhat similar to an associative and coassociative fibrations respectively [84].

We consider the universal moduli spaces of A- and C-cycles, i.e.

$$
\begin{aligned}
\mathcal{M}^{A-c y c l e} & =\coprod_{M \in \tilde{\mathcal{M}}_{G_{2}}} \mathcal{M}^{A-c y c l e}(M) \text { and } \\
\mathcal{M}^{C-\text { cycle }} & =\coprod_{M \in \tilde{\mathcal{M}}_{G_{2}}} \mathcal{M}^{C-\text { cycle }}(M) .
\end{aligned}
$$

There are analogs of the Abel-Jacobi maps to the universal $G_{2}$-intermediate Jacobian,

$$
\begin{aligned}
& A J_{G_{2}}: \mathcal{M}^{A-\text { cycle }} \rightarrow \mathcal{J}_{G_{2}} \text { and } \\
& A J_{G_{2}}: \mathcal{M}^{C-\text { cycle }} \rightarrow \mathcal{J}_{G_{2}},
\end{aligned}
$$

whose images give Lagrangian subspaces in the Kähler manifold $\mathcal{J}_{G_{2}}$. Recall that $\mathcal{J}_{G_{2}}$ has a Lagrangian fibration over $\tilde{\mathcal{M}}_{G_{2}}$. Locally, these Lagrangian subspaces are graphs of exact one forms on $\tilde{\mathcal{M}}_{G_{2}}$ and these functions are $G_{2^{-}}$superpotentials as in the physics literatures.

8.4. $G_{2}$-Donaldson-Thomas bundles. Donaldson and Thomas generalized the ASD connections over oriented four manifolds and introduced the DT-connections on vector bundles $E$ over manifolds with holonomy $S U(3), G_{2}$ and $\operatorname{Spin}(7)$. In the $G_{2}$-setting, the DT-equation for the curvature $F_{A}$ is

$$
* F_{A}+F_{A} \wedge \Omega=0
$$

or equivalently,

$$
F_{A} \wedge(* \Omega)=0 \in \Omega^{6}(M, a d(E)) .
$$


DT-connections are absolute minimizers of the Yang-Mills functional $\int\left|F_{A}\right|^{2}$. We can also rewrite the DT-equation as

$$
F_{A} \in \Omega_{14}^{2}(M, a d(E)) .
$$

Recall that $\mathbf{g}_{2} \subset s o(7)$ corresponds to the component $\Lambda_{14}^{2} \subset \Lambda^{2}$. Globally we have the infinitesimal frame bundle $\mathbf{g}_{2}(M) \cong \Lambda_{14}^{2} T_{M}^{*} \subset \Lambda^{2} T_{M}^{*}$. Thus the above equation is the same as

$$
F_{A} \in \mathbf{g}_{2}(M) \otimes a d(E) .
$$

In this form, the $G_{2}$-DT connections are special $\mathbb{O}$-connections over $M$ from the point of view of geometry over normed division algebra (section 10).

We could consider the previous Chern-Simons type functionals to this setting,

$$
\begin{aligned}
C S_{G_{2}} & : \mathcal{A}(M) \rightarrow \mathbb{R} \\
C S_{G_{2}}\left(D_{A_{1}}\right) & =\int_{[0,1] \times M} \operatorname{Tr} \exp \left(f^{*}(\Omega+* \Omega)+F_{A}\right) \\
& =\int_{[0,1] \times M} \operatorname{Tr}\left[* \Omega \wedge \frac{1}{2} F_{A}^{2}+\frac{1}{4 !} F_{A}^{4}\right] .
\end{aligned}
$$

The Euler-Lagrange equation is a perturbation of the $G_{2}$-DT equation,

$$
F_{A} \wedge * \Omega+\frac{1}{3 !} F_{A}^{3}=0 .
$$

The original $G_{2}$-DT equation is the Euler-Lagrangian equation for the functional $\int_{[0,1] \times M} * \Omega \wedge \operatorname{Tr} F_{A}^{2}$.

The moduli space $\mathcal{M}^{D T}(M)$ of $G_{2}$-DT connections over $M$ has many properties similar to those for moduli of (co-)associative cycles. For instance, it has a natural three form, four form and a cubic tensor, similar to the $G_{2^{-}}$ forms and Yukawa couplings.

8.5. $G_{2}$-dualities, trialities and M-theory. The mystery of the nonassociativity of $\mathbb{O}$ is hidden in the triality and we expect that the full symmetry for $G_{2}$-geometry is a mirror triality.

$G Y Z$ duality. For mirror symmetry, mirror Calabi-Yau threefolds $X$ and $Y$ admit dual special Lagrangian $T^{3}$-fibrations. Furthermore, the mirror transformation between the complex geometry and the symplectic geometry is a certain generalization of the Fourier-Mukai transformation along these $T^{3}$ fibers. Gukov-Yau-Zaslow [58] argues physically that if we couple $X$ with a stable $E_{8} \times E_{8}$-bundle, then its string theory is dual to the M-theory on a $G_{2}$-manifold $M$ with a coassociative K3-fibration. On the fiber level, this is the duality that we mentioned in section 6.4. However, it is not clear how to transform the Calabi-Yau geometry on $X$ to the $G_{2}$-geometry on $M$. One is also curious whether this is part of a triality symmetry among $X, Y$ and $M$. 
Triality flops. When we resolve a rational double point singularity in a Calabi-Yau threefold, we could either smooth it by deforming its complex structure, thus producing a vanishing cycle $S^{3}$, or we could blow it up, i.e. deforming its symplectic structure, and producing an exceptional cycle $S^{2}$. The mirror symmetry for this extremal transition has produced many exciting mathematics. Recall that there are two ways to blowup and they are related by a simple flop. It is expected that the quantum symplectic geometry is unchanged under a simple flop.

Atiyah-Witten [7] discovered that the extremal transition from $S^{2}$ to $S^{3}$ and the flop between $S^{2}$ 's can be naturally unified from the $G_{2}$-perspectives. Namely the $G_{2}$-singular cone $C\left(S^{3} \times S^{3}\right)$ admits three different resolutions, each of them is the spinor bundle $S_{S^{3}}$ over $S^{3}$ and they are related by triality $G_{2}$-flops. Only when we quotient by $S^{1}$, three different resolutions give $T^{*} S^{3}$, $O_{S^{2}}(-1,-1), O_{S^{2}}(-1,-1)$ and they are related by extremal transitions and flop. Hence the dualities for Calabi-Yau threefolds become more symmetric from the $G_{2}$-perspectives. It is natural to ask how the various $G_{2}$-TFTs behave under triality $G_{2}$-flops. For instance, it is interesting to ask whether the skein relation for knot invariants is a consequence of the triality for the associative geometry under a $G_{2}$-flop.

In M-theory, physicists study the membrane theory on an eleven dimensional spacetime $\mathbb{R}^{3,1} \times M$ with $M$ a $G_{2}$-manifold. M-theory reduces to the superstring theory when $M$ is the product of a Calabi-Yau threefold with $S^{1}$ whose size goes to zero. As we mentioned above, there are many duality transformations in M-theory. We hope that they can be combined together to form a mirror triality.

We remark that there is a similar story for eight dimensional Spin (7)manifolds, which is related to F-theory in physics. However we will not further discuss it here.

\section{Geometry of vector cross products}

A Kähler structure on a Riemannian manifold $(M, g)$ is a parallel Hermitian complex structure $J: T_{x} M \rightarrow T_{x} M$. $J$ being a linear Hermitian complex structure is equivalent to

$$
J u \perp u \quad \text { and } \quad|J u|=|u| .
$$

Similarly, a $G_{2}$-structure is a parallel product structure

$$
\times: T_{x} M \otimes T_{x} M \rightarrow T_{x} M
$$

satisfying similar defining properties. Their corresponding symplectic 2-form $\omega$ and $G_{2} 3$-form $\Omega$ play important roles in these geometries. Another example is the standard volume form on $\mathbb{R}^{3}$ gives the vector product, or cross product, $u \times v$ on $\mathbb{R}^{3}$. Such product structures coming from volume forms and Kähler forms have particularly nice properties and they are examples of real vector cross products (abbrev. $\mathbb{R}-\mathrm{VCP}$ ) [85]. VCPs are classified and 
the remaining two are $G_{2}$-VCP and $\operatorname{Spin}(7)$-VCP. Manifolds with these structures play important roles in string theory, M-theory and F-theory.

For $\mathbb{C}-\mathrm{VCP}$, there are only two of such and they come from holomorphic volume forms and holomorphic symplectic forms. The corresponding Kähler manifolds are Calabi-Yau manifolds and hyperkähler manifolds. Thus $\mathbb{R}-$ VCP and $\mathbb{C}$-VCP give a nice uniform description of special holonomies.

\subsection{VCP manifolds.}

Riemannian manifolds with VCP. The classical vector product, or cross product, on $\mathbb{R}^{3}$ satisfies the property that $u \times v$ is perpendicular to both $u$ and $v$ and with length equal to the area of the parallelogram spanned by $u$ and $v$. The only such structure in higher dimension is the $G_{2}$-structure. A real vector cross product is such a product structure but we allow the number $r$ of variables to be different from two. When $r=1$, this is a Hermitian complex structure.

We define an $r$-fold vector cross product ( $\mathbb{R}$-VCP or simply VCP) on a Euclidean space $\mathbb{R}^{m}$ to be a skew-symmetric multi-linear form

$$
\times: \mathbb{R}^{m} \otimes \cdots \otimes \mathbb{R}^{m} \rightarrow \mathbb{R}^{m}
$$

which satisfies

$$
\begin{aligned}
& \text { (i) }\left(u_{1} \times \cdots \times u_{r}\right) \perp u_{i} \quad \text { for all } i \\
& \text { (ii) }\left|u_{1} \times \cdots \times u_{r}\right|=\left\|u_{1} \wedge \cdots \wedge u_{r}\right\| \text {. }
\end{aligned}
$$

Here $\|\cdot\|$ is the norm on $r$-forms.

Similar to the Kähler form and the $G_{2}$-form, we define the VCP-form $\Omega \in \Omega^{r+1}\left(\mathbb{R}^{m}\right)$ as follow

$$
\Omega\left(u_{1}, \ldots, u_{r}, u_{r+1}\right)=\left\langle u_{1} \times \cdots \times u_{r}, u_{r+1}\right\rangle .
$$

The condition (i) for $\times$ ensures that $\Omega$ is skew-symmetric and condition (ii) is equivalent to

$$
\left|\iota_{u_{1}} \iota_{u_{2}} \ldots \iota_{u_{r}} \Omega\right|=1
$$

for any orthonormal vectors $u_{i}$ 's.

It turns out that there are very few possibilities of such and they are classified by Gray [50]: Any VCP-form must be one of the following: (1) volume form, (2) symplectic form, (3) $G_{2}$-form and (4) Spin (7)-form. $G_{2}$ and Spin (7) VCPs live in 7 and 8 dimensional spaces respectively and they are both directly linked to the octonion $\mathbb{O}$. In terms of $\mathbb{R}^{7} \simeq \operatorname{Im} \mathbb{O}$ and $\mathbb{R}^{8} \simeq \mathbb{O}$ and octonion multiplications, we have, in the $G_{2}$-case,

$$
\begin{aligned}
u \times v & =\operatorname{Im} u v \\
\Omega_{G_{2}} & =d x^{123}-d x^{167}+d x^{145}+d x^{257}+d x^{246}-d x^{356}+d x^{347},
\end{aligned}
$$


and in the Spin (7)-case,

$$
\begin{aligned}
u \times v \times w= & (u(\bar{v} w)-w(\bar{v} u)) / 2 \\
\Omega_{\operatorname{Spin}(7)}= & -d x^{1234}-d x^{5678}-\left(d x^{21}+d x^{34}\right)\left(d x^{65}+d x^{78}\right) \\
& -\left(d x^{31}+d x^{42}\right)\left(d x^{75}+d x^{86}\right)-\left(d x^{41}+d x^{23}\right)\left(d x^{85}+d x^{67}\right) .
\end{aligned}
$$

Here $d x^{i j k}$ means $d x^{i} \wedge d x^{j} \wedge d x^{k}$ and so on. Note that $G_{2}$ and $\operatorname{Spin}(7)$ are the symmetry groups of the corresponding VCP-forms in $\mathbb{R}^{7}$ and $\mathbb{R}^{8}$ respectively.

A Riemannian manifold $(M, g)$ with a $V C P$ (resp. closed $V C P$ and parallel $V C P$ ) [85] means that it has $\mathrm{VCP} \times$ on each of its tangent space and $\Omega$ is a smooth form (resp. closed form and parallel form). For simplicity, we usually call a Riemannian manifold with a parallel VCP simply a VCPmanifold.

9.2. Instantons and branes. The closedness of $\Omega$ implies that $\Omega \in$ $\Omega^{r+1}(M)$ is a calibrating form. A $(r+1)$-dimensional submanifold $C$ in $M$ is calibrated by $\Omega$ if and only if it is preserved by $\times$ and they are called instantons. For instance, when $M$ is a Kähler manifold, instantons are holomorphic curves; when $M$ is a $G_{2}$-manifold, instantons are associative submanifolds; when $M$ is a Spin (7)-manifold, instantons are Cayley submanifolds.

The following characterization of an instanton is useful in studying the deformation theory of instantons: First we denote the symmetry group of $\times$ as $G \subset O(m)$ and its Lie algebra as $\mathbf{g} \subset \mathbf{o}(m) \cong \Lambda^{2} V^{*}$. Over a VCPmanifold $M$, by putting $\mathbf{g}$ for each tangent space $V=T_{x} M$ together, we have a subbundle $\mathbf{g}_{M} \subset \Lambda^{2} T_{M}^{*}$. We define a homomorphism

$$
\tau: \Lambda^{r+1} V \rightarrow \Lambda^{2} V
$$

as the composition of the VCP of the last $r$ components follows by the wedge product, i.e.

$$
\tau\left(u_{0} \wedge u_{1} \wedge \cdots \wedge u_{r}\right)=u_{0} \wedge\left(u_{1} \times \cdots \times u_{r}\right) .
$$

The image of $\tau$ lies inside $\mathbf{g}^{\perp}$, the orthogonal complement of the Lie algebra of $G$. Over $M$, we have

$$
\tau \in \Omega^{r+1}\left(M, \mathbf{g}_{M}^{\perp}\right)
$$

and $C$ is a instanton in $M$ if and only if

$$
\left.\tau\right|_{C}=0 \in \Omega^{r+1}\left(C, \mathbf{g}_{M}^{\perp}\right) .
$$

Using this, one can show that infinitesimal deformations of an instanton in $M$ are parametrized by twisted harmonic spinors on $C$.

The well-behaved free boundary condition for holomorphic curves $C$ in a Kähler manifold $C$ is by requiring the boundary $\partial C$ to lie inside a Lagrangian 
submanifold $L \subset M$. This notion generalizes to VCP-geometry. A submanifold $L$ in $M$ is called a brane if (i) $\left.\Omega\right|_{L}=0$ and (ii) $\operatorname{dim} L=(n+r-1) / 2$. Indeed this is the largest possible dimension among submanifolds $L$ satisfying $\left.\Omega\right|_{L}=0$. Lagrangian submanifolds in Kähler manifolds, hypersurfaces in volume manifolds and coassociative submanifolds in $G_{2}$-manifolds are examples of branes. However, brane does not exist in any Spin (7)-manifold.

Suppose $L$ is a brane in $M$, VCP determines a homomorphism from $\Lambda^{r} T_{L}$ to the normal bundle $N_{L / M}$. Taking the adjoint of it, $N_{L / M}$ becomes a subbundle of $\Lambda^{r} T_{L}^{*}$ which is spanned by VCP-forms of degree $r$ on $L$. Infinitesimal deformations of a brane are parametrized by such degree $r$ closed VCP-forms on $L$ and they all have unobstructed deformations. In particular, the moduli space of branes in $M$ is always smooth.

Thus the theory of VCP gives a unified approach to describe the geometries of various important geometric objects in Kähler geometry, $G_{2^{-}}$ geometry and Spin(7)-geometry. The following table summarizes all these structures.

\begin{tabular}{|l||l|l|l|l|}
\hline VCP form & Volume form & Sympl. form & $G_{2}$-form & Spin (7)-form \\
\hline Instanton & Domain & Holo. curve & Assoc. submfd. & Cayley submfd. \\
\hline Brane & Hypersurface & Lagr. submfd. & Coass. submfd. & $\mathrm{n} / \mathrm{a}$ \\
\hline
\end{tabular}

9.3. Symplectic geometry on higher dimensional knot spaces. Symplectic forms are 1-fold VCP forms. Indeed the geometry of $r$-fold VCP on $M$ can be described in terms of the symplectic geometry of the infinite dimensional knot space $\mathcal{K}_{\Sigma}(M)[83]$. Here $\mathcal{K}_{\Sigma}(M)$ is the set of all submanifolds in $M$ which are diffeomorphic to a fixed $(r-1)$-dimensional space $\Sigma$, that is $\mathcal{K}_{\Sigma}(M)=\operatorname{Map}_{\text {emb }}(\Sigma, M) / \operatorname{Diff}(\Sigma)$. Here $\operatorname{Map}(\Sigma, M)$ is the space of all embeddings from a fixed $(r-1)$-dimensional manifold $\Sigma$ to $M$. Via transgression, any $(r+1)$-form $\Omega$ on $M$ defines a 2 -form $\omega_{\mathcal{K}}$ on $\mathcal{K}_{\Sigma}(M)$, i.e.

$$
\omega_{\mathcal{K}}(f)(X, Y)=\int_{\Sigma} \iota_{f_{*}(X) \wedge f_{*}(Y)} \Omega
$$

It is clear that $d \omega_{\mathcal{K}}=0$ if and only if $d \Omega=0$. The Riemannian metric on $M$ defines one on $\mathcal{K}_{\Sigma}(M)$. One can show that $\Omega$ is a $r$-VCP form on $M$ if and only if $\omega_{\mathcal{K}}$ is an $1-\mathrm{VCP}$ form on $\mathcal{K}_{\Sigma}(M)$, in particular a symplectic form.

Furthermore, a submanifold $L$ in $M$ is a brane if and only if $\mathcal{K}_{\Sigma}(L)$ is a Lagrangian submanifold in $\mathcal{K}_{\Sigma}(M)$. Given a holomorphic curve $D$ in $\mathcal{K}_{\Sigma}(M)$, it is a family of submanifolds in $M$, i.e. there is a fiber bundle $\Sigma \rightarrow C \rightarrow D$ and a map $C \rightarrow M$, then $C$ is an instanton in $M$ provided that the curve satisfies a normality condition. Thus the geometry of VCP on $M$ is nothing but the symplectic geometry on $\mathcal{K}_{\Sigma}(M)$. For instance, one would expect to have intersection theory of branes defined using instantons, 
similar to the intersection theory of Lagrangian submanifolds defined using $J$-holomorphic discs.

This interpretation of VCP geometry in terms of symplectic geometry on knot spaces is particularly fruitful for $\mathbb{C}$-VCP.

\section{4. $\mathbb{C}$-VCP geometry.}

Kähler manifolds with $\mathbb{C}$-VCP. We can similarly define a $r$-fold complex vector cross product $(\mathbb{C}-\mathrm{VCP})$ on a Hermitian vector space $\mathbb{C}^{m}[\mathbf{8 5}]$. More precisely, a $\mathbb{C}-\mathrm{VCP}$ form $\Omega \in \Omega^{r+1,0}\left(\mathbb{C}^{m}\right)$ must satisfy

$$
\left|\iota_{u_{1}} \iota_{u_{2}} \ldots \iota_{u_{r}} \Omega\right|=1
$$

for any orthonormal complex vector $u_{i}$ 's. There are only two $\mathbb{C}$-VCPs, namely it must be either (i) a complex volume form or (ii) a complex symplectic form.

Suppose $(M, \omega)$ is Kähler manifold with a holomorphic form $\Omega$ defining a $\mathbb{C}$-VCP, then $\Omega$ is automatically parallel. As a result, a Kähler manifold with a holomorphic $\mathbb{C}$-VCP must be either (i) a Calabi-Yau manifold or (ii) a hyperkähler manifold.

Instantons. Since $\Omega$ is a complex valued differential form, we have a $S^{1}$-family of calibrating forms. Namely for any real number $\theta, \operatorname{Re}\left(e^{i \theta} \Omega\right)$ is a calibrating form and those submanifolds $C$ it calibrates are called instantons with phase $\theta$. Instantons can be characterized as those $(r+1)$-dimensional submanifolds $C$ in $M$ satisfying

$$
\left.\operatorname{Im}\left(e^{i \theta} \Omega\right)\right|_{C}=\left.\omega\right|_{C}=0 .
$$

When $M$ is a Calabi-Yau manifold, then $C$ is a special Lagrangian submanifold with phase $\theta$. When $M$ is a hyperkähler manifold, then $C$ is a $J_{\theta}$-holomorphic curve where $J_{\theta}=(\cos \theta) I+(\sin \theta) K$.

$N$-branes and $D$-branes. There are two types of boundary conditions for instantons: Neumann boundary condition and Dirichlet boundary condition. The corresponding branes are called N-branes and D-branes. A $N$-brane is a real $(n+r-1)$-dimensional submanifold $L$ in $M$ satisfying

$$
\left.\Omega\right|_{L}=0 .
$$

A $D$-brane is a middle dimensional submanifold $L$ in $M$ satisfying

$$
\left.\omega\right|_{L}=\left.\operatorname{Re}\left(e^{i \theta} \Omega\right)\right|_{L}=0 .
$$

As in the real case, N-branes are biggest dimension submanifolds where the restriction of $\Omega$ vanishes. This forces $L$ to be a complex submanifold in $M$.

When $M$ is a Calabi-Yau manifold, a N-brane (resp. D-brane) is a complex hypersurface (resp. special Lagrangian submanifold with phase $\theta+\pi / 2$ ) in $M$. Even though complex hypersurfaces and special Lagrangian submanifolds seems to be very different from each other, both of them correspond to complex Lagrangian submanifolds in the isotropic knot space $\hat{\mathcal{K}}_{\Sigma}(M)$ of $M$ 
(as will be explained below). The difference is they are complex Lagrangian with respect to different complex structures in the twistor family of $\hat{\mathcal{K}}_{\Sigma}(M)$.

When $M$ is a hyperkähler manifold, a N-brane (resp. D-brane) is a $J$-complex Lagrangian submanifold (resp. $J_{\theta+\pi / 2}$-complex Lagrangian submanifold) in $M$. The deformation theory of instantons with boundaries lying in N-branes and D-branes was studied by Schoen's school. The following table summarizes these structures.

\begin{tabular}{|l||l|l|}
\hline C-VCP mfd. & Calabi-Yau mfd. & Hyperkähler mfd. \\
\hline Instanton & SLag. w $/ \theta=0$ & $J$-holomorphic curve \\
\hline D-brane & Cpx. hypersurface & $J$-cpx. Lagr. submfd. \\
\hline N-brane & SLag. w $/ \theta=\pi / 2$ & $I$-cpx. Lagr. submfd. \\
\hline
\end{tabular}

9.5. Hyperkähler geometry on isotropic knot spaces of CY. Recall that the $\mathbb{R}-\mathrm{VCP}$ geometry on $M$ can be interpreted as the symplectic geometry on $\mathcal{K}_{\Sigma}(M)$, the space of submanifolds in $M$, i.e.

$$
\mathcal{K}_{\Sigma}(M)=\operatorname{Map}_{\text {emb }}(\Sigma, M) / \operatorname{Diff}(\Sigma) .
$$

We will simply write $\operatorname{Map}(\Sigma, M)$ for $\operatorname{Map}_{\text {emb }}(\Sigma, M)$. For $\mathbb{C}$-VCP we should complexify this picture and consider the quotient of $\operatorname{Map}(\Sigma, M)$ by the complexification of $\operatorname{Diff}(\Sigma)$, which does not exist! So one tries to replace the complex quotient by the symplectic quotient: Suppose $(M, g, J, \omega)$ is a Kähler manifold with a $\mathbb{C}$-VCP form $\Omega \in \Omega^{r+1,0}(M)$. In order to induce a symplectic form $\omega_{\text {Map }}$ on $\operatorname{Map}(\Sigma, M)$ from $\omega$ on $M$, we need to fix a background volume form $\nu_{\Sigma}$ on $\Sigma$, then

$$
\omega_{M a p}(f)(u, v)=\int_{\Sigma}\left(f^{*} \omega\right)(u, v) \nu_{\Sigma}
$$

for any $f \in \operatorname{Map}(\Sigma, M)$ and any $u, v \in T_{f} \operatorname{Map}(\Sigma, M)=\Gamma\left(\Sigma, f^{*} T_{M}\right)$.

Since we have fixed a background volume form on $\Sigma$, only volume preserving diffeomorphisms of $\Sigma$ preserves $\omega_{M a p}$. The moment map for the $\operatorname{Diff}\left(\Sigma, \nu_{\Sigma}\right)$-action on $\operatorname{Map}(\Sigma, M)$ is

$$
\begin{aligned}
\mu: \operatorname{Map}(\Sigma, M) & \rightarrow \Omega^{1}(\Sigma) / d \Omega^{0}(\Sigma) \\
\mu(f) & =[\alpha]
\end{aligned}
$$

where $f^{*} \omega=d \alpha$ (section 6.2). In particular, $\mu^{-1}(0)$ consists of isotropic embeddings of $\Sigma$ into $M$. However, the symplectic quotient

$$
\operatorname{Map}(\Sigma, M) / / \operatorname{Diff}\left(\Sigma, \nu_{\Sigma}\right)=\mu^{-1}(0) / \operatorname{Diff}\left(\Sigma, \nu_{\Sigma}\right)
$$


is too big as we should take the symplectic quotient by the larger group $\operatorname{Diff}(\Sigma)$. In [83], a natural coisotropic foliation $\mathcal{D}$ on $\mu^{-1}(0)$ is constructed and the quotient space

$$
\hat{\mathcal{K}}_{\Sigma}(M)=\mu^{-1}(0) / \operatorname{Diff}(\Sigma)
$$

is a natural substitute for the nonexisting symplectic quotient $\operatorname{Map}(\Sigma, M) / /$ $\operatorname{Diff}(\Sigma)$, called the isotropic knot space. Furthermore, $\hat{\mathcal{K}}_{\Sigma}(M)$ admits almost complex structures $I, J$ and $K$ satisfying the Hamilton relation and a natural 1-fold $\mathbb{C}$-VCP structure. However, in this infinite dimensional setting, we only know that $\hat{\mathcal{K}}_{\Sigma}(M)$ is a holomorphic symplectic manifold, not hyperkähler.

Thus we have constructed an infinite dimensional holomorphic symplectic manifold $\hat{\mathcal{K}}_{\Sigma}(M)$ from any Calabi-Yau manifold $M$. Analogous to the real situation, a normal $J_{\theta}$-holomorphic curve in $\hat{\mathcal{K}}_{\Sigma}(M)$, where $J_{\theta}=$ $(\cos \theta) I+(\sin \theta) K$, corresponds to an instanton, i.e. a special Lagrangian submanifold with phase $\theta$ in $M$.

More interestingly, (i) if $L$ is a complex hypersurface (i.e. a N-brane) in $M$, then $\hat{\mathcal{K}}_{\Sigma}(L)$ is a $J$-complex Lagrangian submanifold in $\hat{\mathcal{K}}_{\Sigma}(M)$ and (ii) if $L$ is a special Lagrangian submanifold with phase $\theta+\pi / 2$ (i.e. a D-brane with phase $\theta$ ), then $\hat{\mathcal{K}}_{\Sigma}(L)$ is a $J_{\theta+\pi / 2}$-complex Lagrangian submanifold in $\hat{\mathcal{K}}_{\Sigma}(M)$. The reason that $\hat{\mathcal{K}}_{\Sigma}(L)$ also defines a complex Lagrangian in $\hat{\mathcal{K}}_{\Sigma}(M)$ is because it is automatically sitting inside $\mu^{-1}(0)$.

For quaternionic-Kähler manifolds, i.e. $\operatorname{Hol}(M, g) \subseteq S p(n) S p(1)$, there is no well-defined $\mathbb{H}-\mathrm{VCP}$ structure because of the noncommutativity of $\mathbb{H}$. In summary, we have explained the source of reduction to every nonsymmetric Riemannian holonomy groups as $M$ carrying different $\mathbb{A}-\mathrm{VCP}$ with $\mathbb{A} \in\{\mathbb{R}, \mathbb{C}, \mathbb{H}\}$.

\begin{tabular}{|l||l|l|l|l|l|}
\hline & none & $r=n-1$ & $r=1$ & $r=2$ & $r=3$ \\
\hline $\mathbb{R}-\mathrm{VCP}$ & $O(n)$ & $S O(n)$ & $U(n)$ & $G_{2}$ & $\operatorname{Spin}(7)$ \\
\hline $\mathbb{C}-\mathrm{VCP}$ & $U(n)$ & $S U(n)$ & $S p(n)$ & & \\
\hline $\mathbb{H}-\mathrm{VCP}$ & $S p(n) S p(1)$ & & & & \\
\hline
\end{tabular}

\section{Geometry over normed division algebras}

\subsection{Manifolds over normed algebras.}

Normed division algebras. We have seen that Riemannian manifolds $(M, g)$ with special holonomy groups have various algebraic structures on their tangent bundles $T M$. It is not surprising that such structures are related to normed algebras $\mathbb{A}$. We recall that a real algebra $\mathbb{A}$ with a norm $\|\cdot\|$ is a normed algebra if it satisfies the obvious compatibility condition:

$$
\|a b\|=\|a\|\|b\|
$$


for any $a, b \in \mathbb{A}$. Note that a normed algebra is always a division algebra. It is a classical fact that $\mathbb{R}, \mathbb{C}, \mathbb{H}$ and $\mathbb{O}$ form the complete list of normed division algebras. In this section, we will see that different holonomy groups correspond to manifolds being defined over various $\mathbb{A}$ and whether it is $\mathbb{A}$-oriented or not.

Each tangent space of a Riemannian manifold $(M, g)$ certainly has a norm. If it also has an $\mathbb{A}$-module structure, then it will reduce the holonomy group of $M$. For instance, a Riemannian manifold with a parallel $\mathbb{C}$-module structure on its tangent spaces is a Kähler manifold. We can give a unified way to describe all Riemannian holonomy groups and their geometries from this point of view, even including Riemannian symmetric spaces (see section 13). There are many advantages to this. It helps us to discover new links between different kinds of geometries and obtain new results. For example the hard Lefschetz $s l(2, \mathbb{R})$-action for compact Kähler manifolds can be naturally generalized to all holonomy groups in $[\mathbf{9 6}]$ and the results of mean curvature flow for Lagrangian submanifolds in Calabi-Yau manifolds was generalized to hyperlagrangian submanifolds in the $\mathbb{H}$ case in [98]. We could even include conformal geometry in the same arena with the help of Jordan algebras (section 12).

The first issue is $\mathbb{O}$-module does not make sense as $\mathbb{O}$ is not an associative algebra. Using Jordan algebras, one can resolve this problem as long as the (O-dimension is not more than three. In fact, we need to use this and its extension via the magic square to describe exceptional symmetric spaces in terms of two normed division algebras. For the time being, we will restrict to the one dimensional case, i.e. $\mathbb{O}$ itself.

$\mathbb{A}$-manifolds and $\mathbb{A}$-orientations. Now we define a Riemannian $\mathbb{A}$ manifold $M$ to be a Riemannian manifold with its holonomy group lies inside the group $G_{\mathbb{A}}(n)$ of twisted isomorphisms of $\mathbb{A}^{n}$, which is defined as follows: Suppose $V$ is a normed linear $\mathbb{A}$-module of rank $n$. A $\mathbb{R}$-linear isometry $\phi$ of $V$ is called a twisted isomorphism if there exists $\theta \in S O(\mathbb{A})$ such that

$$
\phi(v x)=\phi(v) \theta(x)
$$

for any $v \in V$ and $x \in \mathbb{A}$. When $\mathbb{A}$ is $\mathbb{R}$ or $\mathbb{C}, \theta$ is necessarily the identity. The reason of introducing $\theta$ is because a $\mathbb{H}$-structure is given by the twistor $S^{2}$-family of complex structures, rather than a particular choice of the triple $(I, J, K)$. We have the symmetry group $G_{\mathbb{H}}(n)$ is $S p(n) S p(1)$. In the $\mathbb{O}$ case, $G_{\mathbb{O}}(1)$ is the exceptional holonomy group $\operatorname{Spin}(7)$.

Next we introduce the notion of an $\mathbb{A}$-orientation for Riemannian $\mathbb{A}$-manifolds. Orientability requires the notion of determinant. In the complex and quaternionic cases, $\operatorname{det}_{\mathbb{A}}$ corresponds to the projection to the second component in $U(n)=S U(n) \times U(1) / \mathbb{Z}_{n}$ and $S p(n) S p(1)$ respectively. In the octonion case, it is simply the octonion product because we only consider the one dimensional case. Therefore a twisted isomorphism $g \in G_{\mathbb{A}}(n)$ is called special if $\operatorname{det}_{\mathbb{A}}(g)$ fixes $1 \in \mathbb{A}$. That is $g$ is an element in the isotropic 
subgroup of 1 in $G_{\mathbb{A}}(n)$, which we denote $H_{\mathbb{A}}(n)$ and the corresponding manifolds are called special $\mathbb{A}$-manifolds. They are given explicitly in the following table:

\begin{tabular}{|c||c|c|c|c|}
\hline $\mathbb{A}$ & $\mathbb{R}$ & $\mathbb{C}$ & $\mathbb{H}$ & $\mathbb{O}$ \\
\hline$G_{\mathbb{A}}(n)$ & $O(n)$ & $U(n)$ & $S p(n) S p(1)$ & $\operatorname{Spin}(7)$ \\
\hline$H_{\mathbb{A}}(n)$ & $S O(n)$ & $S U(n)$ & $S p(n)$ & $G_{2}$ \\
\hline
\end{tabular}

Their corresponding Riemannian geometries are as follows.

\begin{tabular}{|c||c|c|c|c|}
\hline $\mathbb{A}$ & $\mathbb{R}$ & $\mathbb{C}$ & $\mathbb{H}$ & $\mathbb{O}$ \\
\hline$G_{\mathbb{A}}(n)$ & Riemannian & Kähler & Quaternionic-Kähler & $\operatorname{Spin}(7)$ \\
\hline$H_{\mathbb{A}}(n)$ & Volume & Calabi-Yau & Hyperkähler & $G_{2}$ \\
\hline
\end{tabular}

In the above definition, a $G_{2}$-manifold is a real eight dimensional manifold. However its universal cover is a Riemannian product manifold $M \times \mathbb{R}$ with $M$ a seven dimensional $G_{2}$-manifold as defined previously. This is because $G_{2}=A u t(\mathbb{O})$ fixes $\mathbb{R} \subset \mathbb{O}$ and therefore sits inside $S O(7)$.

$s u_{\mathbb{A}}(1,1)_{\text {sup }}$-action. When $M$ is a Kähler manifold, the hard Lefschetz theorem says that there is a natural $s l_{2}(\mathbb{R})$-action on differential forms $\Omega^{*}(M)$ and the cohomology group $H^{*}(M, \mathbb{R})$. Notice that $s l_{2}(\mathbb{R}) \cong s u(1,1)$. Indeed there is a super Lie algebra

$$
s u(1,1)_{\text {sup }}=s u(1,1) \oplus \mathbb{C}^{1,1} \oplus \mathbb{R}
$$

which acts on the space of differential forms $\Omega^{*}(M)$ extending the hard Lefschetz action. Here $\mathbb{C}^{1,1}$ acts by the first order differential operators $\partial, \bar{\partial}$, $\partial^{*}$ and $\bar{\partial}^{*}$ and $\mathbb{R}$ acts by the second order differential operator, the Laplacian $\Delta$. Having such an action encompass (i) the hard Lefschetz action, (ii) first order Kähler identities and (iii) second order Kähler identities.

Suppose $M$ is only an oriented Riemannian manifold, then $\Omega^{*}(M)$ admits an $s o(1,1)_{\text {sup }}=s o(1,1) \oplus \mathbb{R}^{1,1} \oplus \mathbb{R}$ where $\mathbb{R}^{1,1}$ acts via $d$ and $d^{*}$ and $\mathbb{R}$ acts via $\Delta$.

When $M$ is a hyperkähler manifold, there is a similar action of

$$
s p(1,1)_{\text {sup }}=s p(1,1) \oplus \mathbb{H}^{1,1} \oplus \mathbb{R}
$$

on $\Omega^{*}(M)$ encompassing all the hyperkähler identities.

The existence of such actions can be explained via the geometry over normed algebras point of view. The basic reason is the exterior algebra $\Lambda^{*}\left(\mathbb{A}^{n}\right)$ can be naturally identified with the spinor representation for the vector space $\mathbb{A}^{n, n}$. Such actions have natural generalization to all ( $\mathbb{A}$-oriented) $\mathbb{A}$-manifolds $[\mathbf{9 3}]$. 
10.2. Gauge theory over (special) $\mathbb{A}$-manifolds. When a Riemannian manifold has special holonomy, there is usually a special kind of connections on bundles over them, for instance holomorphic bundles over Kähler manifolds and Donaldson-Thomas bundles over $G_{2^{-}}$and Spin (7)-manifolds. Using normed division algebras to describe different holonomy groups gives us a particular nice unified way to describe all these different types of connections.

If $M$ is a (special) $\mathbb{A}$-manifold, then the Lie algebra of its holonomy group is $\left(\mathbf{h}_{\mathbb{A}}(n) \subset\right) \mathbf{g}_{\mathbb{A}}(n) \subset \mathbf{s o}(m)$, where $m$ and $n$ are dimensions of $M$ over $\mathbb{R}$ and $\mathbb{A}$ respectively. This determines a subbundle in the bundle of 2 -forms $\Lambda^{2} T_{M}^{*}$, denoted

$$
\mathbf{h}_{\mathbb{A}}\left(T_{M}\right) \subset \mathbf{g}_{\mathbb{A}}\left(T_{M}\right) \subset \Lambda^{2} T_{M}^{*} .
$$

Recall that the curvature $F_{A}$ of a connection $D_{A}$ on a bundle $E$ is a $a d(E)$ valued 2-form, $F_{A} \in \Lambda^{2} T_{M}^{*} \otimes a d(E)$. On a (special) $\mathbb{A}$-manifold $M$, it is natural to require $F_{A}$ to lie inside $\Gamma\left(M, \mathbf{g}_{\mathbb{A}}\left(T_{M}\right) \otimes a d(E)\right)\left(\operatorname{resp} . \Gamma\left(M, \mathbf{h}_{\mathbb{A}}\left(T_{M}\right) \otimes\right.\right.$ $a d(E))$ ) and we call such a connection a (special) $\mathbb{A}$-connection. Most of them can be identified with well-known Yang-Mills connections as indicated in the following table.

\begin{tabular}{|c|c|c|}
\hline & $\mathbb{A}$-connections & Special $\mathbb{A}$-connections \\
\hline $\mathbb{C}$ & $\begin{array}{l}F_{E}^{0,2}=F_{E}^{2,0}=0 \\
\text { (Holomorphic bundles) }\end{array}$ & $\begin{array}{l}F_{E}^{0,2}=F_{E}^{2,0}=\Lambda F=0 \\
\text { (Hermitian Yang-Mills bdls.) }\end{array}$ \\
\hline $\mathbb{H}$ & $\begin{array}{l}F \in \mathbf{g}_{\mathbb{H}}\left(T_{M}\right) \otimes a d(E) \\
(\text { B-bundles })\end{array}$ & $\begin{array}{l}F_{I}^{0,2}=F_{J}^{0,2}=F_{K}^{0,2}=0 \\
\text { (ASD or hyperholomorphic bdls.) }\end{array}$ \\
\hline (1) & $\begin{array}{l}* F_{E}+\Theta \wedge F_{E}=0 \\
(\operatorname{Spin}(7) \text {-Donaldson-Thomas bdls. })\end{array}$ & $\begin{array}{l}F \wedge \Theta=0 \\
\left(G_{2} \text {-Donaldson-Thomas bdls. }\right)\end{array}$ \\
\hline
\end{tabular}

10.3. A-submanifolds and (special) Lagrangian submanifolds.

$\mathbb{A}$-submanifolds in $\mathbb{A}$-manifolds. Complex submanifolds form an important class of submanifolds in Kähler manifolds. However there are very few quaternionic submanifolds in a $\mathbb{H}$-manifold as they are always totally geodesic submanifolds. And we have no $\mathbb{O}$-submanifold as $G_{2^{-}}$and $\operatorname{Spin}(7)$ manifolds already have $\mathbb{O}$-dimension one. However, the geometry of (special) Lagrangian submanifolds in (special) $\mathbb{A}$-manifolds is very rich.

(Special) $\mathbb{A} / 2$-Lagrangian submanifolds. When a symplectic vector space $(V, \omega)$ has a compatible complex structure $J$, a Lagrangian subspace $L$ in $V$ gives an orthogonal decomposition $V=L \oplus J L$. When $V$ is an $\mathbb{A}$-module, it has several compatible complex structures $J_{1}, \ldots, J_{2 r-1}$ where $r=\operatorname{dim}_{\mathbb{R}} \mathbb{A} / 2$ and therefore $V$ has several symplectic structure $\omega_{1}, \ldots, \omega_{2 r-1}$. For example, 
when $V=\mathbb{H}^{n}$, a subspace which is both $\omega_{I^{-}}$and $\omega_{K^{-}}$Lagrangian is automatically $J$-linear and therefore a $J$-complex Lagrangian subspace. In general, if $L \subset \mathbb{A}^{n}$ is Lagrangian with respect to half of the symplectic forms, say $\omega_{1}, \ldots, \omega_{r}$, then it is $J_{l}$-linear for the remaining complex structures. In particular, it is a $(\mathbb{A} / 2)$-module. Here we denote $\mathbb{C} / 2=\mathbb{R}, \mathbb{H} / 2=\mathbb{C}$ and $\mathbb{O} / 2=\mathbb{H}$. We call such a $L$ a $(\mathbb{A} / 2)$-Lagrangian subspace in $V$. Globally on $M$, this defines the notion of $(\mathbb{A} / 2)$-Lagrangian submanifolds in any Riemannian $\mathbb{A}$-manifold.

There is also a corresponding notion of orientability for such $L$, i.e. phase angles are constant on $L$, which defines the class of special $(\mathbb{A} / 2)$-Lagrangian submanifold and all of these are calibrated submanifolds. To describe the phase angle concretely, we recall the Grassmannian of $(\mathbb{A} / 2)$-Lagrangian subspaces in $\mathbb{C}^{n}$ (resp. $\mathbb{H}^{n}$ and $\left.\mathbb{O}\right)$ is $U(n) / S O(n)(\operatorname{resp} . S p(n) S p(1) / U(n)$ $U(1)$ and $\left.\operatorname{Spin}(7) / S p(1)^{3}\right)$. There is phase angle map to $S^{1}$ (resp. $S^{2} / \pm 1$ and $\left.S^{4}\right)$ and the fiber over $\theta$ consists of special $(\mathbb{A} / 2)$-Lagrangian subspaces of phase angle $\theta$. Thus giving any $(\mathbb{A} / 2)$-Lagrangian submanifold $L$, there is a phase angle map

$$
\theta: L \rightarrow S^{d}
$$

where $d=\operatorname{dim} \mathbb{A} / 2$ and in the $\mathbb{H}$-case it is well-defined up to the antipodal map. $L$ is special if this is the constant map.

We identify all the them in the following table.

\begin{tabular}{|c||l|l|}
\hline $\mathbb{A}$ & $\frac{\mathbb{A}}{2}$-Lagrangian submfd. & special $\frac{\mathbb{A}}{2}$-Lagr. submfd. \\
\hline \hline $\mathbb{C}$ & Lagrangian submfd. & special Lagr. submfd. \\
\hline $\mathbb{H}$ & hyperlagrangian submfd. & complex Lagrangian submfd. \\
\hline $\mathbb{O}$ & Cayley submfd. & (co-)associative submfd. \\
\hline
\end{tabular}

When $L$ is a Lagrangian submanifold in a Calabi-Yau manifold $M$, the phase angle function $\theta(x)$ satisfies

$$
\iota_{H} \omega=d \theta(x),
$$

where $H$ is the mean curvature vector of $L$. This implies that Lagrangian submanifolds in $M$, or more generally in a Kähler-Einstein manifold, are invariant under the mean curvature flow. And the phase angle $\theta(x)$ satisfies the non-linear PDE

$$
\frac{\partial \theta}{\partial t}=\Delta \theta
$$

where $\Delta$ is the Laplacian operator with respect to the induced metric on $L$ which is changing it $t$. It does not develop type I singularity. Furthermore, if $L$ is minimal, then it must be calibrated, namely a special Lagrangian submanifold. 
Indeed, the same is true for $\mathbb{A} / 2$-Lagrangian submanifolds in any special $\mathbb{A}$-manifold in general. When $\mathbb{A}=\mathbb{O}$, Cayley submanifold is already calibrated and therefore the other interesting situation is for hyperlagrangian submanifolds $L$ in a hyperkähler manifold $M[\mathbf{9 8}]$. In this case, the phase angle function $\theta(x): L \rightarrow S^{2} / \pm 1$ has the following nice property: The target $S^{2}$ is the twistor family of complex structures on $M$ and for any $x \in L$, the tangent space $T_{x} L \subset T_{x} M$ is complex linear with respect to the complex structure $J_{\theta(x)}$. We also have

$$
\iota_{H} \Omega_{J_{\theta(x)}}=\bar{\partial} \theta,
$$

and $\theta(x)$ satisfies the harmonic map flow. These can be used to show that the mean curvature flow preserves the hyperlagrangian property and type I singularity does not appear, just as what happens in the Calabi-Yau case.

\section{Quaternion geometry}

When we go from $\mathbb{R}$ to $\mathbb{C}$ and then to $\mathbb{H}$, their geometries become more and more rigid. For instance, every higher dimensional $\mathbb{H}$-manifold is Einstein. Also every complex submanifold in a Kähler manifold has zero mean curvature, in fact an absolute volume minimizer, and every quaternionic submanifold has zero second fundamental form, i.e. a totally geodesic submanifold. This is because the second fundamental form must be skew-Hermitian with respect to $I$ and $J$ (of course $K$ as well). Using $I J=K$, it becomes Hermitian with respect to $K$ and therefore it must be zero. In general, every quaternionic map from $\mathbb{H}^{m}$ to $\mathbb{H}^{n}$ must be affine $\mathbb{H}$-linear.

Twistor spaces. Recall that a Riemannian $\mathbb{H}$-manifold $M$ has its holonomy group in $S p(n) S p(1)$. It is also called a quaternionic-Kähler manifold. If we also assume $\mathbb{H}$-orientability, then its holonomy group is inside $S p(n)$ and it is a hyperkähler manifold. Every tangent space $T_{x} M$ is modeled on $\mathbb{H}^{n}$, thus it has three complex structures $I, J$ and $K$. Indeed, any element in the unit sphere $S^{2}$ in the three dimensional vector space spanned by $I, J$ and $K$ is a complex structure on $T_{x} M$. They form a $S^{2}$-fiber bundle

$$
S^{2} \rightarrow Z \rightarrow M \text {. }
$$

The total space is called the twistor space. In fact, this is the associated bundle for the $S p(n) S p(1)$-frame bundle over $M$ via the adjoint action of the $S p(1)$-factor on $S^{2} \subset \operatorname{Im} \mathbb{H}$. Equivalently, the inclusion $S p(n) S p(1) \subset$ $S O(4 n)$ defines a subbundle in the bundle of two forms,

$$
\Lambda_{s p(n)}^{2} \oplus \Lambda_{s p(1)}^{2} \subset \Lambda^{2},
$$

and $Z$ is the unit sphere bundle inside $\Lambda_{s p(1)}^{2}$. When $M$ is of dimension four, the above inclusion is the decomposition of any two form into ASD and SD components,

$$
\Lambda_{-}^{2} \oplus \Lambda_{+}^{2}=\Lambda^{2}
$$


The tangent space of $Z$ at any $\left(x, J_{x}\right)$ in $Z$ splits into vertical and horizontal components via the Riemannian metric on $M$. Since each fiber is $S^{2}$, the vertical component has a natural complex structure and $J_{x}$ is a complex structure on the horizontal component, thus $Z$ has a natural almost complex structure. In fact, it is integrable as long as $n \geq 2$, namely the twistor space is always a complex manifold. Moreover every $S^{2}$ fiber is a holomorphic curve in $Z$. When $n=1$, this requires $W^{+}=0$.

Furthermore, the $\mathbb{H}$-geometry on $M$ can be described in terms of the complex geometry on $Z$, the so-called twistor transformation.

11.1. Hyperkähler geometry. On a hyperkähler manifold $M, \mathbb{H}$ orientibility guarantees that each $I, J$ and $K$ can be consistently defined over the whole manifold $M$. In particular $Z=M \times S^{2}$, but non-holomorphically. Indeed, the projection to $S^{2}$ is the $S^{2}$-family of complex structures on $M$, called the twistor family. The three complex structures satisfies the Hamilton relation,

$$
I^{2}=J^{2}=K^{2}=I J K=-i d .
$$

Given any $J$ in the twistor family, $(M, g, J)$ is a Calabi-Yau manifold which corresponds to the embedding $S p(n) \subset S U(2 n)$, analogous to $U(n) \subset$ $S O(2 n)$ in the complex case. The corresponding Kähler form will be denoted as $\omega_{J}$. The parallel form

$$
\Omega_{J}=\omega_{I}-i \omega_{k} \in \Omega_{J}^{2,0}(M)
$$

defines a holomorphic symplectic structure on $(M, J)$. The $J$-holomorphic volume form for the Calabi-Yau structure on $(M, J)$ is simply the top exterior power of $\Omega_{J}$.

Conversely, if a compact Kähler manifold $(M, J)$ admits a holomorphic symplectic form $\Omega_{J}$, then $T_{M}$ is isomorphic to $T_{M}^{*}$ as holomorphic vector bundles and therefore all its odd Chern classes vanish. In particular, $c_{1}(M)=0$ and it admits a Ricci flat Kähler metric $g$ by Yau's theorem. $\Omega_{J}$ is a parallel form with respect to $g$ and both $\operatorname{Re} \Omega_{J}$ and $\operatorname{Im} \Omega_{J}$ define Kähler structures on $M$ which make $M$ into a hyperkähler manifold.

Examples of hyperkähler manifolds. Using this method, Beauville constructed higher dimensional hyperkähler manifolds from four dimensional ones $S$, i.e. K3 surfaces and Abelian surfaces, by resolving the singularities of symmetric powers of $S$, called the Hilbert schemes of points in $S$. Instead of considering moduli space of points in $S$, Mukai [109] showed that the moduli space $\mathcal{M}$ of stable coherent sheaves on $S$ has a canonical holomorphic symplectic form. When there is no strictly semi-stable sheaf, then $\mathcal{M}$ is compact and therefore a hyperkähler manifold. O'Grady [110] studied the resolutions of the compactification of $\mathcal{M}$ and constructed two compact hyperkähler manifolds which are not birational to Beauville's examples.

Recall that the cotangent bundle of any complex manifold $X$ admits a canonical holomorphic symplectic form but we cannot use Yau's theorem to assert that $T^{*} X$ is hyperkähler as it is noncompact. Nevertheless, 
Calabi $[\mathbf{2 8}]$ has constructed explicit hyperkähler metric on $T^{*} \mathbb{P}^{n}$. In fact, the cotangent bundle of any Hermitian symmetric space of compact type is hyperkähler [14].

Hyperkähler manifolds also arises from the geometry of Calabi-Yau threefolds $Y$. The infinite dimensional space of isotropic knots in $Y$ carries a natural $\mathbb{H}$-structure [83]. Moreover, complex surfaces or special Lagrangian submanifolds in $Y$ define complex Lagrangian submanifolds in it.

As we mentioned in section 7.1, if we enlarge the moduli space of complex structures on Calabi-Yau threefolds by including a choice of the holomorphic volume forms, then the universal intermediate Jacobian over it is a hyperkähler manifold. Moreover, under the Abel-Jacobi map, the universal moduli spaces of holomorphic curves define a complex Lagrangian subvariety in it. The underlying structures on this enlarged moduli space of Calabi-Yau manifolds is the special Kähler structure [43].

Moduli of hyperkähler manifolds. The moduli spaces of hyperkähler manifolds have many nice properties. First since $M$ is always a CalabiYau manifold, the moduli space of complex structures on $M$ is smooth, by the Tian-Todorov lemma. Every complex deformation $M_{t}$ of $M$ is again a hyperkähler manifold and the corresponding holomorphic symplectic form $\Omega_{t}$ satisfies the following important property:

$$
q_{0}\left(\Omega_{t}\right)=0 \text { and } q_{0}\left(\operatorname{Re} \Omega_{t}\right)>0,
$$

Here

$$
\begin{aligned}
q_{0} & : H^{2}(M, \mathbb{C}) \rightarrow \mathbb{C} \\
q_{0}(\phi) & =\frac{n}{2} \int \Omega^{n-1} \bar{\Omega}^{n-1} \phi^{2}+(1-n) \int \Omega^{n-1} \bar{\Omega}^{n} \phi \int \Omega^{n} \bar{\Omega}^{n-1} \phi,
\end{aligned}
$$

is called the Beauville-Bogomolov quadratic form. It is a quadratic form of signature $\left(3, b_{2}-3\right)$ on $H^{2}(M, \mathbb{R})$. The local Torelli theorem says that the period map

$$
\tau: \mathcal{T} \rightarrow \mathcal{D}=S O^{+}\left(3, b_{2}-3\right) / S O(2) S O\left(1, b_{2}-3\right)
$$

which sends a marked hyperkähler manifold $M_{t}$ to the real two plane in $H^{2}(M, \mathbb{R})$ spanned by $\operatorname{Re} \Omega_{t}$ and $\operatorname{Im} \Omega_{t}$ is a local isomorphism.

Complex Lagrangian submanifolds. As we mentioned before, any quaternionic submanifold in a hyperkähler manifold is totally geodesic. Thus there are very few of such. On the other hand, the geometry of complex Lagrangian submanifolds is rather rich. Bryant also studied other types of submanifolds in hyperkähler manifolds $[\mathbf{2 6}][\mathbf{2 1}]$. A complex submanifold $L$ of middle dimension in a holomorphic symplectic manifold $M$ is called a complex Lagrangian submanifold if the restriction of $\Omega_{J}$ to $M$ is zero.

Any middle dimensional complex submanifold $L$ in $M$ with $c_{1}(L)$ positive is always a complex Lagrangian submanifold because there is no nontrivial holomorphic two form on $L$ by Bochner arguments. For instance any $\mathbb{C} \mathbb{P}^{n}$ in $M$ is a complex Lagrangian submanifold. As in the real case, the cotangent 
bundle of any complex manifold $X$ is always a holomorphic symplectic manifold with the zero section $X$ being a complex Lagrangian submanifold in it.

Hitchin showed that when $M$ is a hyperkähler manifold and $L$ is a middle dimensional submanifold in $M$, then the vanishing of $\Omega_{J}$ on $L$ already implies that $L$ is a complex submanifold. On the other hand, a complex submanifold $L$ in $M$ being a complex Lagrangian can be characterized by the property that the cohomology class $[L]$ it represents is $\Omega$-primitive [17]. Furthermore, the rational cobordism class of $L$ is completely determined by $[L] \in H^{2 n}(M, \mathbb{Z})$.

Since complex Lagrangian submanifolds are examples of special Lagrangian submanifolds, the moduli space $\mathcal{M}^{\text {cx Lag }}(M)$ of complex Lagrangian submanifolds in $M$ is smooth. Hitchin [62] defined an analog of the Abel-Jacobi map

$$
\tau: \mathcal{M}^{c x L a g}(M) \rightarrow H^{1}\left(L_{0}, \mathbb{C}\right)
$$

by integrating $\Omega$ along a path of 1 -cycles between any complex Lagrangian submanifold $L$ and a fixed one $L_{0}$. Since $L_{0}$ is a Kähler manifold, $H^{1}\left(L_{0}, \mathbb{R}\right)$ is a complex vector space. Hitchin showed that $\tau$ is a local embedding with image a complex Lagrangian subspace in $H^{1}\left(L_{0}, \mathbb{C}\right) \simeq T^{*}\left(H^{1}\left(L_{0}, \mathbb{R}\right)\right)$. On the cotangent space $T^{*} H^{1}$, there is a canonical pseudo Kähler metric. The restriction of it to $\mathcal{M}^{c x L a g}(M)$ is positive definite and of Hessian type. This implies that $\mathcal{M}^{c x L a g}(M)$ is a special Kähler manifold.

From a physical perspective, the $\sigma$-model with a hyperkähler manifold $M$ as a target has $N=4$ SUSY. If we require the domain Riemann surfaces to have boundaries and their images lie inside a fix complex Lagrangian submanifold $L \subset M$, then only half of the SUSY can be preserved. Hence we have a $N=2$ SUSY theory. Moduli spaces of such theories are generally known (physically) to have special Kähler geometry. In our case here, it is simply the moduli space of complex Lagrangian submanifolds in $M$ (possible with $M$ varying). Therefore we expect such a moduli space is special Kähler.

Complex Lagrangian fibrations. There are many examples of hyperkähler manifolds that admit complex Lagrangian fibrations. For instance, the moduli space of curves coupled with line bundles in any K3 surface $S$ has a complex Lagrangian fibration with each fiber consisting of sheaves which support on a fixed curve in $S$. This moduli space plays an important role in the original derivation of the Yau-Zaslow formula (section 6.4). Every elliptic fibration on a K3 surface $S$ is a complex Lagrangian fibration because of the low dimension. This induces complex Lagrangian fibrations on the Hilbert schemes of points in $S$.

Matsushita [106] proved that every holomorphic fibration on an irreducible projective hyperkähler manifold is a complex Lagrangian fibration provided that the base is normal. The base must be a Fano variety with the same Hodge number as $\mathbb{C P}^{n}$ (or simply write $\mathbb{P}^{n}$ ). A folklore conjecture says that the base must always be $\mathbb{P}^{n}$. Note that Huybrechts proved that 
given any compact hyperkähler manifold $M$, a given complex structure is projective if and only if there exists a holomorphic line bundle $L$ over $M$ such that $q_{0}\left(c_{1}(L)\right)>0$ where $q_{0}$ is the Beauville-Bogomolov quadratic form.

For a complex Lagrangian fibration on a projective hyperkähler manifold, every smooth fiber is an Abelian variety. We call such a fibration an algebraically integrable system. In this case, the base has a natural special Kähler structure away from the discriminant locus. Furthermore, the second fundamental forms for the fibers define a symmetric cubic tensor on the base which characterizes this fibration, as studied by Donagi-Markman [34][35].

Hyperkähler flops. There are explicit examples of hyperkähler metrics on cotangent bundles of any compact Hermitian symmetric space, for instance the complex Grassmannian $G r_{\mathbb{C}}(r, n)$ or $\mathbb{P}^{n}$. Using the fact that the cotangent bundles of a vector space $V$ and its dual space $V^{*}$ are both equal to $V \times V^{*}$, there is a canonical identification between $T^{*} \mathbb{P}^{n}$ and $T^{*}\left(\mathbb{P}^{n}\right)^{*}$ outside their zero sections $\mathbb{P}^{n}$ and $\mathbb{P}^{n *}$ respectively. This surgery operator can be done on any holomorphic symplectic manifold $M$ of complex dimension $2 n$. Namely if $M$ contains a complex (Lagrangian) submanifold $\mathbb{P}^{n}$, then we can replace it by $\mathbb{P}^{n *}$ and obtain a new holomorphic symplectic manifold $M^{\prime}$. This is called a hyperkähler flop.

Since $M \backslash \mathbb{P}^{n}$ and $M^{\prime} \backslash \mathbb{P}^{n *}$ are isomorphic, every complex Lagrangian $L$ in $M$ determines one in $M^{\prime}$, called the Legendre transform of $L$, denoted $L^{\vee}$. Note that their intersections with $\mathbb{P}^{n}$ and $\mathbb{P}^{n *}$ are dual varieties. The usual Plücker formula for dual varieties was generalized in $[\mathbf{9 2}]$ and give

$$
L_{1} \cdot L_{2}+\frac{\left(L_{1} \cdot \mathbb{P}^{n}\right)\left(L_{2} \cdot \mathbb{P}^{n}\right)}{(-1)^{n+1}(n+1)}=L_{1}^{\vee} \cdot L_{2}^{\vee}+\frac{\left(L_{1}^{\vee} \cdot \mathbb{P}^{n *}\right)\left(L_{2}^{\vee} \cdot \mathbb{P}^{n *}\right)}{(-1)^{n+1}(n+1)} .
$$

We recall the classical Plucker formula for an algebraic curve $C \subset \mathbb{P}^{2}$ and its dual curve $C^{\vee} \subset \mathbb{P}^{2 *}$,

$$
d^{\vee}=d(d-1)-2 \delta-3 \kappa,
$$

where $d\left(\right.$ resp. $d^{\vee}$ ) is the degree of $C$ (resp. $C^{\vee}$ ) and $\delta$ (resp. $\kappa$ ) is the number of double points (resp. cusp) of $C$.

Thus this gives a duality transformation between the categories of coherent sheaves on complex Lagrangians submanifolds in $M$ and its hyperkähler flop $M^{\prime}$.

When the $\mathbb{H}$-dimension of $M$ is two, $\mathrm{Hu}$ and Yau [68] showed that hyperkähler flops are the only birational transformations among hyperkähler manifolds. Note that birational transformations are isomorphisms in $\mathbb{H}-$ dimension one. For higher dimensional hyperkähler manifolds, Mukai [109] studied a hyperkähler flop along a family of $\mathbb{P}^{k}$ in $M$ when the total space $Z$ of the family is a complex coisotropic submanifold in $M$ of complex codimension $k$. This is also called a Mukai elementary modification. There is also a natural generalization of the Legendre transformation between categories of complex Lagrangians in this setting [92]. 
More general birational transformations can be constructed when the above coisotropic family of $\mathbb{P}^{k}$ develops singularities. Then the model birational transformation between $T^{*} \mathbb{P}^{n}$ and $T^{*} \mathbb{P}^{n *}$ will be replaced by Springer correspondences, for instance between cotangent bundles of complex Grassmannians $T^{*} G r_{\mathbb{C}}\left(r, \mathbb{C}^{n}\right)$ and $T^{*} G r_{\mathbb{C}}\left(r, \mathbb{C}^{n *}\right)$. This stratified version of the Mukai elementary transformation is constructed by Markman.

11.2. Quaternionic-Kähler geometry. Oriented four manifolds are quaternionic-Kähler manifolds of $\mathbb{H}$-dimension one, because $S p(1) S p(1)=$ $S O$ (4). We usually referred to quaternionic-Kähler manifolds $M$ as those with holonomy group equals $S p(n) S p(1)$ instead of a subgroup of it. When $n \geq 2$, quaternionic-Kähler manifolds have two very special properties: (1) the Ricci curvature of $M$ equals $g$ or $-g$ and they are called positive and negative respectively. Namely they are Einstein manifolds. (2) The canonical almost complex structure on the twistor space $Z$ is always integrable, i.e. $Z$ becomes a complex manifold. As a result, we usually require oriented four manifolds to have these properties to be called quaternionic-Kähler, namely they are ASD Einstein four manifolds.

The list of compact Riemannian symmetric spaces which are quaternionic-Kähler is

$$
\begin{gathered}
\frac{S p(n)}{S p(n-1) S p(1)}, \frac{U(n)}{U(n-2) U(2)}, \frac{O(n)}{O(n-4) O(4)}, \frac{G_{2}}{S p(1) S p(1)}, \\
\times \frac{F_{4}}{S p(3) S p(1)}, \frac{E_{6}}{S U(6) S p(1)}, \frac{E_{7}}{S p i n(12) S p(1)}, \frac{E_{8}}{E_{7} S p(1)} .
\end{gathered}
$$

Notice that for each simply connected compact Lie group, there is exactly one compact quaternionic-Kähler symmetric space associated to it.

It has been a folklore conjecture that these are the only quaternionicKähler manifolds in the positive case and this has recently been proven by Kobayashi.

\section{Conformal geometry}

Two Riemannian metrics $g$ and $g^{\prime}$ on $M$ are called conformally equivalent if there is a smooth function $u$ on $M$ such that $g^{\prime}=e^{2 u} g$. A conformal structure on $M$ is an equivalence class of Riemannian metrics $g$ under conformal equivalences. For example, the standard metric on $S^{n} \backslash\{p\}$ is conformally equivalent to $\mathbb{R}^{n}$ and we called such a metric conformally flat.

The following combination of the curvature tensor depends only on the conformal class of $g$,

$$
W=R m-\frac{1}{n-2}\left(R c-\frac{R}{n} g\right) \circ g-\frac{R}{2 n(n-1)} g \circ g,
$$

and $W$ is called the Weyl curvature tensor. When $\operatorname{dim} M \geq 4, g$ is locally conformally flat if and only if $W=0$. When $\operatorname{dim} M=2, M$ is a Riemann 
surface and conformal structures are the same as complex structures on $M$. In particular, the group Conf $\left(S^{2}\right)$ of conformal transformations of $S^{2}$ is infinite dimensional. However, when $n=\operatorname{dim} M \geq 3$, we have

$$
\operatorname{Conf}\left(S^{n}\right)=S O(n+1,1) \text {. }
$$

This group is generated by $S O(n)$ together with inversions.

There are many important topics in conformal geometry, for instances (i) the Yamabe problem, solved by Schoen, says that there is always a constant scalar curvature metric within any conformal class of metrics; (ii) the harmonic energy $E(f)=\int_{\Sigma}|d f|^{2}$ for a map $f:\left(\Sigma, g_{\Sigma}\right) \rightarrow\left(M, g_{M}\right)$ is invariant under conformal change of $g_{\Sigma}$ when $\operatorname{dim} \Sigma=2$. This fact is essential to the two dimensional conformal field theory (abbrev. 2d CFT) in physics (section 3.7); (iii) the Yang-Mills energy functional $\mathcal{Y} \mathcal{M}\left(D_{A}\right)=\int_{M}\left|F_{A}\right|^{2}$ for a connection $D_{A}$ on a unitary bundle $\mathbb{C}^{r} \rightarrow E \rightarrow\left(M, g_{M}\right)$ is invariant under conformal changes of $g_{M}$ when $\operatorname{dim} M=4$. This fact is important for the Donaldson theory (section 4.1). Other conformally invariant quantities include the Willmore functional in dimension four and the conformal volume of Li-Yau.

In this section, we will only explain that conformal geometry can be interpreted as an extension of real, complex, quaternion and octonion geometry with the help of Jordan algebras [79].

Jordan algebras. Due to the nonassociativity of the octonions, we do not have a well-defined notion of its modules $\mathbb{O}^{n}$. Nonetheless, when $n \leq 3$ there is a way to define the symmetry group of the non-existing object $\mathbb{O}^{n}$, by considering the space of self-adjoint operators.

Recall on $\mathbb{R}^{n}$, the space of self-adjoint operators is simply the space of symmetric $n \times n$ matrices, denoted by $S_{n}(\mathbb{R})$. The symmetrization of ordinary matrix multiplication

$$
A \circ B=(A B+B A) / 2
$$

makes $S_{n}(\mathbb{R})$ into a formally real Jordan algebra. Namely it is an algebra over $\mathbb{R}$ whose multiplication $\circ$ is commutative and power associative (that is, $(a \circ a) \circ a=a \circ(a \circ a))$, together with

$$
a_{1} \circ a_{1}+\cdots+a_{n} \circ a_{n}=0 \Rightarrow a_{1}=\cdots=a_{n}=0 .
$$

The same product also makes the space $S_{n}(\mathbb{A})$ of Hermitian symmetric matrices with entries in $\mathbb{A} \in\{\mathbb{R}, \mathbb{C}, \mathbb{H}\}$ into a Jordan algebra. When $n=3$, an analog of the product can still be defined for $\mathbb{A}=\mathbb{O}$, making $S_{3}(\mathbb{O})$ into an exceptional Jordan algebra (see e.g. [9]) even though $\mathbb{O}$ lacks of associativity.

Inside $S_{n}(\mathbb{A})$ we may collect all rank one projections, which are matrices $p$ with $p \circ p=p$ and $\operatorname{tr} p=1$, to form the projective space $\mathbb{A P}^{n-1}$. For instance, even though one has problem to define $\mathbb{O}^{3}$, each rank one projection operator in $S_{3}(\mathbb{O})$ can be interpreted as an octonion line in $\mathbb{O}^{3}$, and the space of them forms the octonion projective plane $\mathbb{O P}^{2}$, which can be identified as the symmetric space $F_{4} / \operatorname{Spin}(9)$. 
Since $S_{n}(\mathbb{A})$ is the space of self-adjoint operators on $\mathbb{A}^{n}$, it should share the same automorphism group $H_{\mathbb{A}}(n)$ as $\mathbb{A}^{n}$. This is indeed true in the classical cases when $\mathbb{A} \in\{\mathbb{R}, \mathbb{C}, \mathbb{H}\}$ and continues to have such an interpretation in the exceptional case $\mathbb{A}=\mathbb{O}$. The following gives a complete list of simple formally real Jordan algebras and their automorphism groups:

\begin{tabular}{|c||c|c|c|c|c|}
\hline $\mathbb{A}$ & $\mathbb{R}$ & $\mathbb{C}$ & $\mathbb{H}$ & $\mathbb{O}$ & $\mathbb{R}^{m}$ \\
\hline$S_{n}(\mathbb{A})$ & $S_{n}(\mathbb{R})$ & $S_{n}(\mathbb{C})$ & $S_{n}(\mathbb{H})$ & $S_{3}(\mathbb{O})$ & $S_{2}\left(\mathbb{R}^{m}\right) \simeq \mathbb{R}^{m} \oplus \mathbb{R}^{1,1}$ \\
\hline $\mathbb{A} \mathbb{P}^{n-1}$ & $\mathbb{R P}^{n-1}$ & $\mathbb{C P}^{n-1}$ & $\mathbb{H}^{n-1}$ & $\mathbb{O P}^{2}$ & $\mathbb{A} \mathbb{P}^{1}=\mathbb{S}^{m}$ \\
\hline$H_{\mathbb{A}}(n)$ & $S O(n)$ & $S U(n)$ & $S p(n)$ & $F_{4}$ & $S O(m+1)$ \\
\hline
\end{tabular}

Amazingly there is one more item in the list of Jordan algebras besides those coming from normed division algebras, namely the spin factor $S_{2}$ $\left(\mathbb{R}^{m}\right) \simeq \mathbb{R}^{m} \oplus \mathbb{R}^{1,1}$. It consists of $2 \times 2$ matrices of the form

$$
\left(\begin{array}{cc}
a-b & v \\
v & a+b
\end{array}\right) \leftrightarrow\left(\begin{array}{l}
v \\
b \\
a
\end{array}\right)
$$

where $v \in \mathbb{R}^{m}$ and $a, b \in \mathbb{R}$, and we set $v \cdot w=v^{t} w$ for $v, w \in \mathbb{R}^{m}$ to carry out matrix multiplication. The embedded projective space is

$$
\left\{\left(\begin{array}{c}
v \\
b \\
\frac{1}{2}
\end{array}\right):\|v\|^{2}+b^{2}=\frac{1}{4}\right\} \cong \mathbb{S}^{m} .
$$

Notice that the automorphism group $S O(m+1)$ of $\mathbb{S}^{m}$ is contained as a maximal compact subgroup in the non-compact group $\operatorname{Conf}\left(\mathbb{S}^{m}\right)=$ $S O(m+1,1)$. A natural question arises: For $\mathbb{A} \in\{\mathbb{R}, \mathbb{C}, \mathbb{H}, \mathbb{O}\}$, is there a symmetry group of $\mathbb{A} \mathbb{P}^{n-1}$ which gives an analog to the conformal symmetry $S O(m+1,1)$ of $\mathbb{S}^{m}$ ?

To answer this question, one identifies $\mathbb{S}^{m}$ as the conformal boundary of the hyperbolic ball

$$
B^{m+1}:=\left\{M \in S_{2}\left(\mathbb{R}^{m}\right): \operatorname{det} M=1\right\} \cong S O(m+1,1) / S O(m+1)
$$

on which $S O(m+1,1)$ acts as isometries. Under this identification, one has $\operatorname{Conf}\left(\mathbb{S}^{m}\right) \cong \operatorname{Isom}\left(B^{m+1}\right)=S O(m+1,1)$ which preserves collinearity in the sense that $\operatorname{Conf}\left(\mathbb{S}^{m}\right)$ maps circles to circles in $\mathbb{S}^{m}$.

Now for $\mathbb{A} \in\{\mathbb{R}, \mathbb{C}, \mathbb{H}\}$, if we collect the symmetries of $\mathbb{A} \mathbb{P}^{n-1}$ which are linear but not necessarily isometries, we obtain the group $S L(n, \mathbb{A})$. Analogously $\mathbb{A} \mathbb{P}^{n-1}$ can be identified as a part of the conformal boundary of $\{M \in$ $\left.S_{n}(\mathbb{A}): \operatorname{det} M=1\right\} \cong S L(n, \mathbb{A}) / S U(n, \mathbb{A})$ on which $S L(n, \mathbb{A})$ acts as isometries. We get the answer for $\mathbb{A} \in\{\mathbb{R}, \mathbb{C}, \mathbb{H}\}: S L(n, \mathbb{A})$ can be regarded as the 
conformal symmetry of $\mathbb{A P}^{n-1}$, which plays the same role as $S O(m+1,1)$ acting on $\mathbb{S}^{m}$. In general, let's denote these symmetry groups as $N_{\mathbb{A}}(n)$ which are listed below. Notice that $H_{\mathbb{A}}(n)$ sits inside $N_{\mathbb{A}}(n)$ as a maximal compact subgroup.

\begin{tabular}{|c||c|c|c|c|c|}
\hline $\mathbb{A}$ & $\mathbb{R}$ & $\mathbb{C}$ & $\mathbb{H}$ & $\mathbb{O}$ & $\mathbb{R}^{m}$ \\
\hline$N_{\mathbb{A}}(n)$ & $S L(n, \mathbb{R})$ & $S L(n, \mathbb{C})$ & $S L(n, \mathbb{H})$ & $E_{6}^{-26}$ & $S O(m+1,1)$ \\
\hline$H_{\mathbb{A}}(n)$ & $S O(n)$ & $S U(n)$ & $S p(n)$ & $F_{4}$ & $S O(m+1)$ \\
\hline
\end{tabular}

Here $E_{6}^{-26}$ is a split Lie group of type $E_{6}$ and its maximum compact subgroup is $F_{4}$.

We observe that when $m=1,2,4$ and $8, N_{\mathbb{R}^{m}}(2)=S L(2, \mathbb{A})$ with $\mathbb{A}$ being real, complex, quaternion and octonion respectively. Hence, $s l(2, \mathbb{R})=$ so $(2,1)$, sl $(2, \mathbb{C})=$ so $(3,1)$, sl $(2, \mathbb{H})=s o(5,1)$, sl $(2, \mathbb{O})=$ so $(9,1)$. In general we have $\operatorname{sl}(2, \mathbb{A})=$ so $\left(\mathbb{A} \oplus \mathbb{R}^{1,1}\right)[\mathbf{9}]$.

The above point of view integrates conformal geometry with real, complex, quaternionic and octonionic geometries.

\section{Geometry of Riemannian symmetric spaces}

13.1. Riemannian symmetric spaces. A Riemannian manifold ( $M$, $g$ ) is called a Riemannian symmetric space, or simply a symmetric space, if every point $x \in M$ has an involutive isometry $\sigma_{x} \in \operatorname{Diff}(M, g)$ satisfying $\sigma_{x}(x)=x$ and $d \sigma_{x}=-i d$ on $T_{x} M$. Recall that involutive means $\sigma_{x}^{2}=i d$. A typical example is the Grassmannian $G r_{\mathbb{R}}(r, n)$ of $r$-dimensional linear subspaces in $\mathbb{R}^{n}$. Suppose $x$ represents a subspace $P_{0}$, then $\sigma_{x}(P)$ is the reflection of $P$ along $P_{0}$ with respect to the orthogonal decomposition $\mathbb{R}^{n}=$ $P_{0} \oplus P_{0}^{\perp}$. Similarly, if $\mathbb{R}^{n}$ is endowed with a complex (resp. symplectic) structure, then the Grassmannian of complex (resp. Lagrangian) subspaces in $\mathbb{R}^{n}$ is also a symmetric space for the same reason. We will explain in this section that all compact Riemannian symmetric spaces are basically of these types.

A noncompact example of a symmetric space is the Grassmannian $G r_{\mathbb{R}}^{+}(r, n)$ of spacelike subspaces, namely it is the set of $r$-dimensional subspaces $P$ in $\mathbb{R}^{r, n-r}$ such that restriction of the signature $(r, n-r)$ inner product $\langle\cdot, \cdot\rangle^{\prime}=\sum_{j=1}^{r}\left(d x^{j}\right)^{2}-\sum_{k=r+1}^{n}\left(d x^{k}\right)^{2}$ to $P$ is positive definite. Note that $G r_{\mathbb{R}}^{+}(r, n)$ is naturally an open subset in $G r_{\mathbb{R}}(r, n)$ and they are called the compact/noncompact dual to each other. Similarly, the Grassmannian of spacelike complex (resp. Lagrangian) subspaces in $\mathbb{C}^{r, n-r}$ is a noncompact symmetric space, denoted as $G r_{\mathbb{C}}^{+}(r, n)\left(\right.$ resp. $L G r_{\mathbb{C}}^{+}(n)$ when $\left.n=2 r\right)$.

The curvature tensor of a symmetric space is always covariant constant, i.e. $\nabla R m=0$. This is because $d \sigma_{x}$ acts as $(-1)$ on $T_{x} M$ and $\nabla R m$ is an odd degree tensor. Conversely, the universal cover of any Riemannian manifold 
$M$ with $\nabla R m=0$ is a symmetric space and we call such a $M$ a locally symmetric space.

A symmetric space $M$ has a large group of isometries $\operatorname{Diff}(M, g)$ which acts transitively on $M$. That is, for any $p, q \in M$, there is an isometry $\sigma$ satisfying $\sigma(p)=q$. Indeed $\sigma$ is the involutive symmetry $\sigma_{x}$ for the midpoint $x$ of the geodesic segment joining $p$ and $q$. If we denote $G=\operatorname{Diff}(M, g)$ and $K$ as the isotropy subgroup fixing a point $x \in M$, then we have

$$
M \cong G / K \text {. }
$$

In particular, $d \sigma_{x}(x)=-i d$ on $T_{x} M=\mathbf{g} / \mathbf{k}$, where $\mathbf{g}$ and $\mathbf{k}$ are the Lie algebras of $G$ and $K$ respectively. Indeed there is a Lie algebra homomorphism $\sigma: \mathbf{g} \rightarrow \mathbf{g}$ satisfying $\sigma^{2}=i d$ and the eigenspace decomposition of $\sigma$ is given by $\mathbf{g}=\mathbf{k} \oplus \mathbf{p}$ with eigenvalues 1 and -1 on $\mathbf{k}$ and $\mathbf{p}$ respectively. Equivalently, $\mathbf{g}$ has a decomposition $\mathbf{g}=\mathbf{k} \oplus \mathbf{p}$ as vector spaces satisfying

$$
[\mathbf{k}, \mathbf{k}] \subset \mathbf{k},[\mathbf{k}, \mathbf{p}] \subset \mathbf{p} \text { and }[\mathbf{p}, \mathbf{p}] \subset \mathbf{k} .
$$

Note that $\mathbf{p}=T_{x} M$. Furthermore, up to covering, $M$ is determined by such a structure $(\mathbf{g}, \sigma)$, called an involutive Lie algebra.

The Riemannian structure on $M$ is reflected by the existence of a positive inner product on $\mathbf{g}$ which is invariant under $\sigma$ and $a d_{\mathbf{g}} \mathbf{k}$ and this is called an orthogonal structure on $(\mathbf{g}, \sigma)$.

The curvature of $M$ can be expressed in terms of the Lie algebra structure of $\mathbf{g}$, namely for any $X, Y \in \mathbf{p}=T_{x} M$, we have

$$
\begin{aligned}
\langle R m(X, Y) X, Y\rangle_{M} & =-\langle[X,[X, Y]], Y\rangle_{\mathbf{g}} \\
R c(X, Y) & =-\operatorname{Tr}_{\mathbf{p}}\left(a d_{X} \circ a d_{Y}\right)=-\frac{1}{2}\langle X, Y\rangle_{\mathbf{g}} .
\end{aligned}
$$

Orthogonal involutive Lie algebras and therefore Riemannian symmetric spaces has been completely classified by Cartan and we will describe them as Grassmannians. We recall from section 3 that holonomy groups of Riemannian manifolds are classified by Berger into two types: the first type are manifolds defined over a normed algebra $\mathbb{A}$ and with or without $\mathbb{A}$-orientation; the second type are locally symmetric spaces.

Suppose $M=G / K$ is an irreducible symmetric space, then it is compact (resp. noncompact) if and only if its curvature is positive (resp. negative), with the only exception that the universal cover of $M$ is $\mathbb{R}^{n}$ in which case its curvature is zero. As the curvature of $M$ can be expressed in terms of the Killing form of $\mathbf{g}, M$ is compact if and only if $G$ is a compact Lie group and $M$ is noncompact if and only if $K$ is a maximal compact subgroup in a noncompact Lie group $G$.

Symmetric spaces always come in pairs $\left(M_{c}, M_{n}\right)$ with $M_{c}=G_{c} / K_{c}$ (resp. $M_{n}=G_{n} / K_{n}$ ) is a compact (resp. noncompact) symmetric space and the Lie algebras have the same complexification

$$
\mathbf{g}_{c} \otimes \mathbf{C} \cong \mathbf{g}_{n} \otimes \mathbf{C},
$$


and under this isomorphism,

$$
\mathbf{k}_{c} \cong \mathbf{k}_{n} \text { and } \quad \mathbf{p}_{c} \cong i \mathbf{p}_{n} .
$$

$M_{c}$ and $M_{n}$ are called compact/noncompact dual to each other. In many cases, $M_{n}$ is naturally an open subset in $M_{c}$. For instance

$$
\frac{O(r, n-r)}{O(r) O(n-r)} \subset \frac{O(n)}{O(r) O(n-r)}
$$

or equivalently,

$$
G r_{\mathbb{R}}^{+}(r, n) \subset G r_{\mathbb{R}}(r, n)
$$

as the open subset consisting of those $r$-dimensional subspaces which are spacelike.

Every compact Lie group $G$ is an example of symmetric spaces when we view it as a quotient of $G \times G$ by $G$ under the diagonal action. The Lie group $O(n)$ (resp. $U(n)$ and $S p(n)$ ) can be viewed as the Grassmannian of maximal isotropic subspaces in $\mathbb{R}^{n, n}$ (resp. $\mathbb{C}^{n, n}$ and $\mathbb{H}^{n, n}$ ). Recall that a maximal isotropic subspace is a half dimensional linear subspace with the restriction of the signature $(n, n)$ inner product to it being identically zero.

When a Lie group $G$ is noncompact, it also determines a symmetric space $M=G / K$ where $K$ is a maximal compact subgroup in $G$. Indeed every noncompact symmetric space is of this form. For example, when $G$ is the complexification $K^{\mathbb{C}}$ of a compact Lie group $K$, then $K^{\mathbb{C}} / K$ is the noncompact dual to $K$.

13.2. Jordan algebras and magic square. Every compact Lie group is a compact symmetric space. Up to finite covers, they are determined by their Lie algebras and they are classified into $A_{n}=s u(n+1), B_{n}=$ so $(2 n+1), C_{n}=s p(n), D_{n}=s o(2 n), E_{6}, E_{7}, E_{8}, F_{4}$ and $G_{2}$. Note that classical Lie algebras, i.e. $A_{n}, B_{n}, C_{n}$ and $D_{n}$, consist of infinitesimal symmetries of inner product spaces $\mathbb{R}^{n}, \mathbb{C}^{n}$ and $\mathbb{H}^{n}$. Recall that $G_{2}$ can also be interpreted as the symmetry group of $\mathbb{O}$. The remaining ones can be interpreted as the symmetries of $(\mathbb{A} \otimes \mathbb{B})^{3}$ with both $\mathbb{A}$ and $\mathbb{B}$ normed division algebras. However, this magic square approach of Tits [123] and Freudenthal $[\mathbf{4 4}]$ is more subtle (see [9] for a beautiful account). They show that

$$
\mathfrak{g}_{n}(\mathbb{A}, \mathbb{B})=\operatorname{Der} S_{n}(\mathbb{A}) \oplus\left[S_{n}(\mathbb{A})^{T r=0} \otimes \operatorname{Im} \mathbb{B}\right] \oplus \operatorname{Der}(\mathbb{B})
$$

admits a natural Lie algebra structure. Here $S_{n}(\mathbb{A})$ is the Jordan algebra of $\mathbb{A}$-Hermitian $n \times n$ matrices ( $n=3$ if $\mathbb{A}$ or $\mathbb{B}$ is the octonion). On the one hand, going from $\mathbb{A}$ to $2 \mathbb{A}$, for instance from $\mathbb{R}$ to $\mathbb{C}$, corresponds to enlarging the symmetry of a linear space $V$ to its complexification $V \oplus V=V \otimes \mathbb{C}$ with one added complex structure. We can also identify this as the tangent bundle $T V$ of $V$. On the other hand, going from $\mathbb{B}$ to $2 \mathbb{B}$ corresponds to enlarging the symmetry of $V$ to its symplectification $V \oplus V^{*}=T^{*} V$. The 
corresponding Lie groups $G_{n}(\mathbb{A}, \mathbb{B})$ (up to finite cover and center) are given in the following table.

\begin{tabular}{|l||l|l|l|l|}
\hline $\mathbb{B} \backslash \mathbb{A}$ & $\mathbb{R}$ & $\mathbb{C}$ & $\mathbb{H}$ & $\mathbb{O}$ \\
\hline \hline $\mathbb{R}$ & $S O(n)$ & $S U(n)$ & $S p(n)$ & $F_{4}$ \\
\hline $\mathbb{C}$ & $S U(n)$ & $S U(n)^{2}$ & $S U(2 n)$ & $E_{6}$ \\
\hline $\mathbb{H}$ & $S p(n)$ & $S U(2 n)$ & $S O(4 n)$ & $E_{7}$ \\
\hline $\mathbb{O}$ & $F_{4}$ & $E_{6}$ & $E_{7}$ & $E_{8}$ \\
\hline
\end{tabular}

This is called the magic square because of the symmetry of interchanging $\mathbb{A}$ and $\mathbb{B}$, just like mirror symmetry! Loosely speaking, $G_{n}(\mathbb{A}, \mathbb{B})$ is the group of symmetries of $(\mathbb{A} \otimes \mathbb{B})^{n}$.

The set of all real linear subspaces in $(\mathbb{A} \otimes \mathbb{B})^{n}$ which are complex linear with respect to all complex structures coming from $\mathbb{A}$ and $\mathbb{B}$ will be called $(\mathbb{A}, \mathbb{B})$-Grassmannian, or simply Grassmannian, and it is denoted as $G r_{\mathbb{A} \mathbb{B}}(r, n)$, or symbolically as $\left\{(\mathbb{A} \otimes \mathbb{B})^{r} \subset(\mathbb{A} \otimes \mathbb{B})^{n}\right\}$ and they are given in the following table.

\begin{tabular}{|c||c|c|c|c|}
\hline $\mathbb{A} \backslash \mathbb{B}$ & $\mathbb{R}$ & $\mathbb{C}$ & $\mathbb{H}$ & $\mathbb{O}$ \\
\hline \hline $\mathbb{R}$ & $\frac{\mathrm{O}(n)}{\mathrm{O}(k) \mathrm{O}(n-k)}$ & $\frac{\mathrm{U}(n)}{\mathrm{U}(k) \mathrm{U}(n-k)}$ & $\frac{\mathrm{Sp}(n)}{\mathrm{Sp}(k) \mathrm{Sp}(n-k)}$ & $\frac{F_{4}}{\mathrm{Spin}(9)}$ \\
\hline $\mathbb{C}$ & $\frac{\mathrm{U}(n)}{\mathrm{U}(k) \mathrm{U}(n-k)}$ & $\frac{\mathrm{U}(n)^{2}}{\mathrm{U}(k)^{2} \mathrm{U}(n-k)^{2}}$ & $\frac{\mathrm{U}(2 n)}{\mathrm{U}(2 k) \mathrm{U}(2 n-2 k)}$ & $\frac{E_{6}}{\mathrm{Spin}(10) \mathrm{U}(1)}$ \\
\hline $\mathbb{H}$ & $\frac{\mathrm{Sp}(n)}{\mathrm{Sp}(k) \mathrm{Sp}(n-k)}$ & $\frac{\mathrm{U}(2 n)}{\mathrm{U}(2 k) \mathrm{U}(2 n-2 k)}$ & $\frac{\mathrm{O}(4 n)}{\mathrm{O}(4 k) \mathrm{O}(4 n-4 k)}$ & $\frac{E_{7}}{\mathrm{Spin}(12) \mathrm{Sp}(1)}$ \\
\hline $\mathbb{O}$ & $\frac{F_{4}}{\mathrm{Spin}(9)}$ & $\frac{E_{6}}{\mathrm{Spin}(10) \mathrm{U}(1)}$ & $\frac{E_{7}}{\mathrm{Spin}(12) \operatorname{Sp}(1)}$ & $\frac{E_{8}}{\mathrm{SO}(16)}$ \\
\hline
\end{tabular}

Every $G r_{\mathbb{A B}}(r, n)$ is a compact Riemannian symmetric space $G / K$ with $G=$ $G_{n}(\mathbb{A}, \mathbb{B})$ and $K$ equals to $G_{r}(\mathbb{A}, \mathbb{B}) G_{n-r}(\mathbb{A}, \mathbb{B})$, possibly up to $U(1)$ or $S p(1)$ factors.

Next we consider real linear subspaces in $(\mathbb{A} \otimes \mathbb{B})^{n}$ which are complex linear with respect to all complex structures coming from $\mathbb{A}$ and $\mathbb{B}$ except the last $J$ from $\mathbb{A}$ and instead we require it to be Lagrangian with respect to $\omega_{J}$. The set of all such subspaces is called Lagrangian $(\mathbb{A}, \mathbb{B})$-Grassmannian, or simply Lagrangian Grassmannian, and it is denoted as $\operatorname{LGr}_{\mathbb{A B}}(n)$, or symbolically as $\left\{\left(\frac{\mathbb{A}}{2} \otimes \mathbb{B}\right)^{n} \subset(\mathbb{A} \otimes \mathbb{B})^{n}\right\}$ and they are given in the following table. 


\begin{tabular}{|l||l|l|l|l|}
\hline $\mathbb{B} \backslash \mathbb{A}$ & $\mathbb{R}$ & $\mathbb{C}$ & $\mathbb{H}$ & $\mathbb{O}$ \\
\hline \hline $\mathbb{R}$ & $\mathrm{n} / \mathrm{a}$ & $\mathrm{n} / \mathrm{a}$ & $\mathrm{n} / \mathrm{a}$ & $\mathrm{n} / \mathrm{a}$ \\
\hline $\mathbb{C}$ & $\frac{U(n)}{O(n)}$ & $\frac{S U(n)^{2}}{S U(n)}$ & $\frac{S U(2 n)}{S p(n)}$ & $\frac{E_{6}}{F_{4}}$ \\
\hline $\mathbb{H}$ & $\frac{S p(n)}{U(n)}$ & $\frac{U(2 n)}{U(n)^{2}}$ & $\frac{S O(4 n)}{U(2 n)}$ & $\frac{E_{7}}{E_{6} U(1)}$ \\
\hline $\mathbb{O}$ & $\frac{F_{4}}{S p(3) S p(1)}$ & $\frac{E_{6}}{S U(6) S p(1)}$ & $\frac{E_{7}}{S p i n(12) S p(1)}$ & $\frac{E_{8}}{E_{7} S p(1)}$ \\
\hline
\end{tabular}

Each $L G r_{\mathbb{A B}}(n)$ is a compact Riemannian symmetric space $G / K$ with $G=$ $G_{n}(\mathbb{A}, \mathbb{B})$ and $K$ equals to $G_{n}\left(\frac{\mathbb{A}}{2}, \mathbb{B}\right)$, possibly up to $U(1)$ or $S p(1)$ factors. This is because the orthogonal complement of a Lagrangian subspace is naturally its dual space. Note that the symmetric space $G_{2} / S O(4)=$ $G_{2} / S p(1)^{2}$ is the Lagrangian Grassmannian $L G r_{\mathbb{O} \mathbb{R}}(1)$. With a little more work, $S O(2 n) / U(n)$ can be described in a similar fashion [69].

In fact we have given the list of all symmetric spaces of compact type up to covering with the exceptions of $E_{6} / S p(4)$ and $E_{7} / S U(8)$. They can be realized as follows: We consider real linear subspaces in $(\mathbb{A} \otimes \mathbb{B})^{3}$ which are complex linear with respect to all complex structures coming from $\mathbb{A}$ and $\mathbb{B}$ except the last complex structures $J_{\mathbb{A}}$ from $\mathbb{A}$ and $J_{\mathbb{B}}$ from $\mathbb{B}$ and instead we require it to be Lagrangian with respect to both $\omega_{J_{\mathbb{A}}}$ and $\omega_{J_{\mathbb{B}}}$. The set of all such subspaces is called double Lagrangian $(\mathbb{A}, \mathbb{B})$-Grassmannian, or simply double Lagrangian Grassmannian, and it is denoted as $L L G r_{\mathbb{A B}}$, or symbolically as $\left\{\Lambda^{2}\left(\frac{\mathbb{A}}{2} \otimes \frac{\mathbb{B}}{2}\right)^{4} \subset(\mathbb{A} \otimes \mathbb{B})^{3}\right\}$ and they are given in the following table.

\begin{tabular}{|c||c|l|l|l|}
\hline $\mathbb{B} \backslash \mathbb{A}$ & $\mathbb{R}$ & $\mathbb{C}$ & $\mathbb{H}$ & $\mathbb{O}$ \\
\hline \hline $\mathbb{R}$ & $\mathrm{n} / \mathrm{a}$ & $\mathrm{n} / \mathrm{a}$ & $\mathrm{n} / \mathrm{a}$ & $\mathrm{n} / \mathrm{a}$ \\
\hline $\mathbb{C}$ & $\mathrm{n} / \mathrm{a}$ & $\frac{S U(3)^{2}}{S O(4)}$ & $\frac{S U(6)}{S U(4)}$ & $\frac{E_{6}}{S p(4)}$ \\
\hline $\mathbb{H}$ & $\mathrm{n} / \mathrm{a}$ & $\frac{S U(6)}{S U(4)}$ & $\frac{S O(12)}{U(6)}$ & $\frac{E_{7}}{S U(8)}$ \\
\hline $\mathbb{O}$ & $\mathrm{n} / \mathrm{a}$ & $\frac{E_{6}}{S p(4)}$ & $\frac{E_{7}}{S U(8)}$ & $\frac{E_{8}}{S O(16)}$ \\
\hline
\end{tabular}

Each $L L G r_{\mathbb{A B}}$ is a compact Riemannian symmetric space $G / K$ with $G=$ $G_{3}(\mathbb{A}, \mathbb{B})$ and $K=G_{4}\left(\frac{\mathbb{A}}{2}, \frac{\mathbb{B}}{2}\right)$ ! A reason for having dimension four appearing in $K$ is roughly as follows: If we consider the decomposition of two forms on $\mathbb{R}^{4}$ into self-dual and anti-self-dual forms, $\Lambda^{2} \mathbb{R}^{4}=\Lambda_{+}^{2} \mathbb{R}^{4} \oplus \Lambda_{-}^{2} \mathbb{R}^{4}$, then 
each $\Lambda_{ \pm}^{2} \mathbb{R}^{4}$ is a Lagrangian subspace in a copy of $\mathbb{C}^{3}$ in the decomposition $\mathbb{C}^{3} \otimes \mathbb{C}=\mathbb{C}^{3} \oplus \mathbb{C}^{3}$. Thus $S U(3)^{2} / S O(4)=[S U(3) / S O(3)]^{2}$ and similarly we have $S U(6) / S U(4)=S U(6) / S O(6)$. We should warn our readers that much care is always needed whenever $\mathbb{A}$ or $\mathbb{B}$ equals $\mathbb{O}[69]$.

These give a simple description of all locally symmetric spaces of compact type up to covering as Grassmannians of complex/Lagrangian subspaces in $(\mathbb{A} \otimes \mathbb{B})^{n}$ except compact Lie groups $G$. In fact compact Lie groups $G_{n}(\mathbb{A}, \mathbb{B})$ can also be described as the Grassmannians of maximal isotropic subspaces in $T^{*}(\mathbb{A} \otimes \mathbb{B})^{n}$ with respect to the canonical symmetric bilinear form of type $(m, m)$. These structures are related to the generalized complex structures as introduced by Hitchin.

How about symmetric spaces $\hat{M}$ of noncompact type? In many cases $\hat{M}$ admits an open embedding into its compact dual $\hat{M} \subset M$. The simplest example is

$$
G r_{\mathbb{R}}^{+}(r, n)=\frac{O(r, n-r)}{O(r) O(n-r)} \subset \frac{O(n)}{O(r) O(n-r)}=G r_{\mathbb{R}}(r, n)
$$

where $G r_{\mathbb{R}}^{+}(r, n)$ is the Grassmannian of spacelike linear subspaces of dimension $r$ in $\mathbb{R}^{r, n-r}$. Indeed this holds true in general for Hermitian symmetric spaces as well.

\subsection{Hermitian and quaternionic symmetric spaces.}

Hermitian symmetric spaces. A Riemannian symmetric space $G / K$ is called a Hermitian symmetric space if it admits a $G$-invariant complex structure. This happens precisely when $K$ has a $U(1)$-factor, possibly up to a finite cover. Classical irreducible compact Hermitian symmetric spaces are classified into

(i) Type I $(\mathrm{p}, \mathrm{q}) S U(p+q) / S(U(p) U(q))$,

(ii) Type II(n) $\operatorname{Spin}(2 n) / U(n)$,

(iii) Type III(n) $S p(n) / U(n)$ and

(iv) Type IV(n) Spin $(n+1) / \operatorname{Spin}(n) U(1)$.

There are natural embeddings

$$
G_{n} / K \subset \mathbf{p} \subset G_{c} / K
$$

where the first embedding realizes $G_{n} / K$ as a bounded domain in $\mathbb{C}^{N}$, called the Harish-Chandra embedding and $G_{n} / K \subset G_{c} / K$ is called the Borel embedding. An example of these embeddings is

$$
G r_{\mathbb{C}}^{+}(r, n) \subset \operatorname{Hom}\left(\mathbb{C}^{r}, \mathbb{C}^{n-r}\right) \subset G r_{\mathbb{C}}(r, n) .
$$

Conversely, every bounded domain $D$ in $\mathbb{C}^{N}$ has a natural Kähler metric, called the Bergman metric. If it is also symmetric in the sense that every point $x \in D$ is fixed by a biholomorphic involution as an isolated fixed point, then this involution must preserve the Bergman metric. Thus noncompact Hermitian symmetric spaces are the same as bounded symmetric domains. 
The bounded symmetric domain $S p(2 n, \mathbb{R}) / U(n)$ can be transformed to the Siegel upper half space $S_{n}^{+}(\mathbb{R})+i S_{n}(\mathbb{R})$ by the Cayley transformation, where $S_{n}^{+}(\mathbb{R})$ is the set of positive definite symmetric matrices of rank $n$. It is well-known that $S p(2 n, \mathbb{R}) / U(n)$ is the universal cover of the moduli space of $n$-dimensional Abelian varieties, or equivalently the moduli space of weight one polarized Hodge structures. Indeed every noncompact Hermitian symmetric space can be described as the moduli space of polarized Hodge structures of certain types by the work of Deligne.

Recall that $S_{n}(\mathbb{R})$ is a Jordan algebra and every Jordan algebra is of the form $S_{n}(\mathbb{A})$, with $\mathbb{A} \in\left\{\mathbb{R}, \mathbb{C}, \mathbb{H}, \mathbb{O}, \mathbb{R}^{m}\right\}$. When $\mathbb{A}=\mathbb{O}$, we have $n=3$ and $S_{3}(\mathbb{O})$ is the exceptional Jordan algebra; when $\mathbb{A}=\mathbb{R}^{m}$, we have $n=2$ and $S_{2}\left(\mathbb{R}^{m}\right) \cong \mathbb{R}^{m, 1}$ is the spin factor. Every $S_{n}^{+}(\mathbb{A})+i S_{n}(\mathbb{A})$ is a noncompact Hermitian symmetric space and they form the complete list of tube domains. We have

\begin{tabular}{|c||c|c|c|c|c|}
\hline $\mathbb{A}$ & $\mathbb{R}$ & $\mathbb{C}$ & $\mathbb{H}$ & $\mathbb{O}$ & $\mathbb{R}^{m}$ \\
\hline \hline$S_{n}^{+}(\mathbb{A})+i S_{n}(\mathbb{A})$ & $\frac{\mathrm{Sp}(2 n, \mathbb{R})}{\mathrm{U}(n)}$ & $\frac{\mathrm{U}(n, n)}{\mathrm{U}(n)^{2}}$ & $\frac{\mathrm{O}^{*}(4 n)}{\mathrm{U}(2 n)}$ & $\frac{\mathrm{E}_{7,3}}{\mathrm{E}_{6} \mathrm{U}(1)}$ & $\frac{\mathrm{O}(m+2,2)}{\mathrm{O}(m+2) \mathrm{O}(2)}$ \\
\hline
\end{tabular}

In fact, they are simply the Grassmannians of spacelike $(\mathbb{C} \otimes \mathbb{A})$-Lagrangians in $(\mathbb{H} \otimes \mathbb{A})^{n}$,

$$
S_{n}^{+}(\mathbb{A})+i S_{n}(\mathbb{A}) \cong L G r_{\mathbb{H} \mathbb{A}}^{+}(n) .
$$

We also remark that $S_{n}^{+}(\mathbb{A}) \cong L G r_{\mathbb{C A}}^{+}(n)$.

Quaternionic symmetric spaces. A symmetric space $M=G / K$ is called a quaternionic symmetric spaces if it is also a quaternionic-Kähler manifold, namely its holonomy group lies inside $S p(n) S p(1)$ with $4 n=\operatorname{dim}_{\mathbb{R}} M$. Similar to the characterization of Hermitian symmetric spaces, $G / K$ is a quaternionic symmetric space if and only if $K$ contains a $S p(1)$-factor, possibly up to a finite cover. This $S p$ (1)-factor always corresponds to the longest root of $G$. As a matter of fact, there is exactly one compact quaternionic symmetric space for each simple Lie group, up to finite covers.

Classical compact quaternionic symmetric spaces are

$$
\frac{S p(n+1)}{S p(n) S p(1)}, \frac{U(n+2)}{U(2) U(2)} \text { and } \quad \frac{O(n+4)}{O(n) O(4)} .
$$

Note that $S p(n+1) / S p(n) S p(1) \cong \mathbb{H} \mathbb{P}^{n}$ is the set of $\mathbb{H}$-lines in $\mathbb{H}^{n+1}$. Recall from the magic square that when $n=2 m, \mathbf{s u}(2 m)$ should be regarded as the set of infinitesimal symmetries of $(\mathbb{C} \otimes \mathbb{H})^{m}$. Thus $U(n+2) / U(n)$ $U(2) \cong(\mathbb{C} \otimes \mathbb{H}) \mathbb{P}^{m}$. Similarly, $O(n+4) / O(n) O(4) \cong(\mathbb{H} \otimes \mathbb{H}) \mathbb{P}^{m}$ with $n=4 m$. We can also include the exceptional symmetric space $(\mathbb{O} \otimes \mathbb{H}) \mathbb{P}^{2} \cong$ $E_{7} / S O(12) O(4)$ in this list of quaternionic symmetric spaces of the form $(\mathbb{A} \otimes \mathbb{H}) \mathbb{P}^{m}$ for some normed division algebra $\mathbb{A}$. 
Exceptional compact quaternionic symmetric spaces are

$$
\frac{F_{4}}{S p(3) S p(1)}, \frac{E_{6}}{S U(6) U(2)}, \frac{E_{7}}{S O(12) O(4)}, \frac{E_{8}}{E_{7} S p(1)} \text { and } \frac{G_{2}}{S p(1)^{2}} \text {. }
$$

The first four in the above list correspond to the sets of $\mathbb{H}$-Lagrangians in $(\mathbb{O} \otimes \mathbb{A})^{3}$ with $\mathbb{A}=\mathbb{R}, \mathbb{C}, \mathbb{H}$ and $\mathbb{O}$ respectively, i.e. $L G r_{\mathbb{O A}}(3)$. And the last one is simply the set of $\mathbb{H}$-Lagrangians in $\mathbb{O}$, i.e. $L G_{\mathbb{H}}(1)$.

\section{Conclusions}

In this article, we summarized certain aspects of geometric structures on Riemannian manifolds $M$. From a topological perspective, we discussed bundles and submanifolds, leading to K-theory and cohomology theory. When $M$ is equipped with a Riemannian metric, we look for canonical structures by minimizing the energy of a connection and the volume of a submanifold. The Euler-Lagrange equations are the Yang-Mills equations and the minimal surface equations. They are both system of second order partial differential equations.

Most spaces we encounter in nature have more geometric structures. That is why symmetry is such an important subject in physics. Spaces with the largest amount of symmetries are Riemannian symmetric spaces. They are model spaces in geometry as well as many important moduli spaces.

A compatible complex structure $J$ on $M$ gives a symplectic structure $\omega$ via $\omega(u, v)=g(J u, v)$. This makes $M$ into a Kähler manifold. Kähler geometry is a very rich subject as it includes the complex algebraic geometry for projective manifolds. Kählerian is also the necessary and sufficient condition for the existence of $N=2$ supersymmetry for $\sigma$-models in physics. $J$ defines the complex geometry on $M$ while $\omega$ defines the symplectic geometry on $M$. These two geometries are conjecturally dual to each other by the mirror symmetry conjecture, provided that $M$ has a $\mathbb{C}$-orientation, namely a Calabi-Yau manifold.

Berger classified all possible reduced holonomy groups and the author described them in terms of normed algebras $\mathbb{A} \in\{\mathbb{R}, \mathbb{C}, \mathbb{H}, \mathbb{O}\}$ and $\mathbb{A}$ orientability. This means that geometric structures on $(M, g)$ do always come from, possibly more than one, $J$ or equivalently $\omega$. This approach also gives a unified description of various canonical Yang-Mills connections and calibrated submanifolds in $M$. As a result, we found new results and relationships among these geometries. These canonical connections and submanifolds are not just critical points of the energy/volume functionals, they are absolute minimizers. Furthermore they are governed by first order PDEs and they define, at least formally, topological invariants of $M$ via various topological field theories.

The $\mathbb{O}$-geometry has the richest structure and its corresponding geometries have holonomy groups $\operatorname{Spin}(7) \supset G_{2} \supset S U(3)$ in dimension 8, 7 and 6 respectively. The lack of associativity for $\mathbb{O}$ is rescued by the triality as we 
have seen it reflected in $G_{2}$-geometry. The octonion structure is also equivalent to the vector cross product, which is a natural generalization of the complex structure, thus we have seen that $G_{2}$-geometry resembles the Kähler geometry in many ways. The $\mathbb{H}$-Lagrangian geometry in the $\mathbb{O}$-geometry brings the beautiful low dimensional geometry and physics in dimension 4, 3 and 2 into an integral part of the $\mathbb{O}$-geometry. For instance the author expects that the skein relation for knots in $\mathbb{R}^{3}$ and the Morgan-MrowkaSzabó formula for the SW-invariants are consequences of the $G_{2}$-triality.

The duality and triality transformations among different geometries have proven to be a powerful tools to uncover many structures and producing amazing formulas. Many of these formulas have been verified by computational methods. The SYZ approach to explain the mirror symmetry has sparked many exciting developments in mathematics, as well as in physics. We expect to continue to see many more of such developments in the coming years until we fully understand the SYZ transformations.

\section{References}

[1] B. S. Acharya and B. Spence, Supersymmetry and M theory on 7-manifolds, [hepth/0007213].

[2] P. Aspinwall, K3 Surfaces and String Duality, in C. Esthimiou and B. Greene, editors, "Fields, Strings and Duality, TASI 1996", pages 421-540, World Scientific, 1997.

[3] M. Atiyah, New invariants of 3- and 4-dimensional manifolds. The mathematical heritage of Hermann Weyl (Durham, NC, 1987), 285-299, Proc. Sympos. Pure Math., 48, Amer. Math. Soc., Providence, RI, 1988.

[4] M. Atiyah, N. Hitchin and I.M. Singer, Self-duality in four-dimensional Riemannian geometry. Proc. Roy. Soc. London Ser. A 362 (1978), no. 1711, 425-461.

[5] M. Atiyah, V. Patodi, and I.M. Singer, Spectral asymmetry and Riemannian geometry, I, Math. Proc. Camb. Phil. Soc 77 (1975), 43-69.

[6] M. Atiyah and I.M. Singer, The index of elliptic operators. I. Ann. of Math. (2) 87 (1968) 484-530.

[7] M. Atiyah and E. Witten, $M$-theory dynamics on a manifold of $G_{2}$-holonomy. Adv. Theor. Math. Phys. 6 (2002), no. 1, 1-106.

[8] D. Auroux, Mirror symmetry and T-duality in the complement of an anticanonical divisor. arXiv:0706.3207.

[9] J.C. Baez, The octonions, Bull. Amer. Math. Soc. (N.S.) 39 (2002), no. 2, 145-205.

[10] A. Beauville, Counting rational curves on K3 surfaces. Duke Math. J. 97 (1999) no. 1, 99-108.

[11] M. Berger, Sur les groupes d'holonomie des variétés à connexion affine et des variétés riemanniennes, Bull. Soc. Math. France 83, (1955), 279-330.

[12] M. Bershadsky, S. Cecotti, H. Ooguri and C. Vafa, Kodaira-Spencer theory of gravity and exact results for quantum string amplitudes. Comm. Math. Phys. 165 (1994), no. 2, 311-427.

[13] A. Besse, Einstein manifolds. Ergebnisse der Mathematik und ihrer Grenzgebiete (3) 10. Springer-Verlag, Berlin, 1987.

[14] O. Biquard and P. Gauduchon, Hyperkahler metrics on cotangent bundles of Hermitian Symmetric Spaces, in Geometry and physics, edited by Andersen et. (1997) p. $287-298$. 
[15] A. Bondal and D. Orlov, Derived categories of coherent sheaves. Proceedings of the International Congress of Mathematicians, Vol. II (Beijing, 2002), 47-56, 2002.

[16] R.B. Brown and A.Gray, Vector Cross Products, Comment. Math. Helv. 42 (1967), $222-236$.

[17] J. Bryan, S. Katz and N.C. Leung, Multiple covers and the integrality conjecture for rational curves in Calabi-Yau threefolds, Journal of Algebraic Geometry, 10, no. 3, (2001), 549-568.

[18] J. Bryan and N.C. Leung, Generating functions for the number of curves on abelian surfaces. Duke Math. J., 99(2):311-328, 1999.

[19] J. Bryan and N.C. Leung, The enumerative geometry of K3 surfaces and modular forms. J. Amer. Math. Soc. 13(2000) no. 2, 371-410.

[20] J. Bryan and N.C. Leung, Counting curves on irrational surfaces. Surveys in differential geometry: differential geometry inspired by string theory, 313-339, Surv. Differ. Geom., 5, Int. Press, Boston, MA, 1999.

[21] R. Bryant, Submanifolds and special structures on the octonians. J. Differential Geom. 17 (1982), no. 2, 185-232.

[22] R. Bryant, Metrics with exceptional holonomy, Ann. of Math. 126 (1987), 525-576.

[23] R. Bryant, A survey of Riemannian metrics with special holonomy groups. Proceedings of the International Congress of Mathematicians, Vol. 1, 2 (Berkeley, Calif., 1986), 505-514, Amer. Math. Soc., Providence, RI, 1987.

[24] R. Bryant, Recent advances in the theory of holonomy. Seminaire Bourbaki, Vol. 1998/99. Asterisque No. 266 (2000), Exp. No. 861, 5, 351-374.

[25] R. Bryant and P. Griffiths, Some observations on the infinitesimal period relations for regular threefolds with trivial canonical bundle. Arithmetic and geometry, Vol. II, 77-102, Progr. Math., 36, Birkhauser Boston, Boston, MA, 1983.

[26] R. Bryant and R. Harvey, Submanifolds in hyper-Kahler geometry. J. Amer. Math. Soc. 2 (1989), no. 1, 1-31.

[27] R. Bryant and S. Salamon, On the construction of some complete metrics with exceptional holonomy, Duke Math. J. 58, no.3 (1989) 829.

[28] E. Calabi, Metriques Kahleriennes et fibres holomorphes, Ann. Ec. Norm. Sup. 12 (1979) 269-294.

[29] P. Candelas, X.C. de la Ossa, P.S. Green, L. Parkes, A pair of Calabi-Yau manifolds as an exactly soluble superconformal theory. Nucl. Phys., B 359 (1991), 21-74.

[30] D. Cox and S. Katz, Mirror symmetry and algebraic geometry. Mathematical Surveys and Monographs, 68. American Mathematical Society, Providence, RI, 1999.

[31] K.W. Chan and N.C. Leung, On SYZ mirror transformations. math.SG0808.1551.

[32] K.W. Chan and N.C. Leung, Mirror symmetry for toric Fano manifolds via $S Y Z$ transformations. arXiv:0801.2830

[33] R. Dijkgraaf, S. Gukov, A. Neitzke and C. Vafa, Topological M-theory as Unification of Form Theories of Gravity. HUTP-04/A042, ITFA-2004-54. Adv.Theor.Math.Phys. 9:603-665,2005.

[34] R. Donagi, E. Markman, Spectral covers, algebraically completely integrable, Hamiltonian systems, and moduli of bundles, in Lecture Notes in Math. vol. 1620 (Springer, 1996) pp. 1-119 [alg-geom/9507017].

[35] R. Donagi, E. Markman, Cubics, integrable systems, and Calabi-Yau threefolds. Proc. Hirzebruch Conf., Israel Math. Conf. Proc. 9(1996), 199-221. [alg-geom/9408004].

[36] S. Donaldson, Anti self-dual Yang-Mills connections over complex algebraic surfaces and stable vector bundles. Proc. London Math. Soc. (3) 50 (1985), no. 1, 1-26.

[37] S. Donaldson, Moment Maps and Diffeomorphisms Asian J. Math. Vol 2. No. 1, $1-16$.

[38] S. Donaldson and P. Kronheimer, The geometry of four-manifolds. Oxford Mathematical Monographs. Oxford Science Publications. The Clarendon Press, Oxford University Press, New York, 1990. x +440 pp. 
[39] S. Donaldson and E. Segal, Gauge Theory in higher dimensions, II. arXiv:0902.3239.

[40] S. Donaldson and R. P. Thomas, Gauge Theory in Higher Dimensions, The Geometric Universe (Oxford, 1996), Oxford Univ. Press, Oxford (1998) pp. 31-47.

[41] J. Eells and J.H. Sampson, Harmonic mappings of Riemannian manifolds. Amer. J. Math. 86 (1964) 109-160.

[42] A. Floer, Morse theory for Lagrangian intersections. J. Differential Geom. 28 (1988), no. 3, 513-547.

[43] D. Freed, Special Kahler manifolds, C.M.P. 203 (1999) 31-52.

[44] H. Freudenthal, Beziehungen der $E_{7}$ und $E_{8}$ zur Oktavenebene, I, Proc. Kon. Ned. Ak. v. Wet., 57 (1954), 218-230; VIII, ibid., 62 (1959), 447-465.

[45] K. Fukaya, Y.G. Oh, H. Ohta and K. Ono, Lagrangian intersection Floer theory anomoly and obstruction, International Press.

[46] G. Gibbons, D. Page and C. Pope, Einstein metrics on $S^{3}, \mathbb{R}^{3}$ and $\mathbb{R}^{4}$ bundles, Comm. Math. Phys. 127 (1990) 529.

[47] A.B. Givental, Homological geometry and mirror symmetry, Proc. ICM. Vol. 2 Birkhauser (1995) 472-480.

[48] R. Gopakumar and C. Vafa, M-theory and Topological Strings-II, [hep-th/9812127].

[49] L. Göttsche, A conjectural generating function for numbers of curves on surfaces. Comm. Math. Phys. 196 (1998), no. 3, 523-533.

[50] A. Gray, Vector cross products on manifolds, Trans. Amer. Math. Soc. 141 (1969) $465-504$.

[51] B. Greene, A. Shapere, C. Vafa and S.-T.Yau, Stringy cosmic strings and noncompact Calabi-Yau manifolds. Nuclear Phys. B 337 (1990), no. 1, 1-36.

[52] M. Gross, Special Lagrangian fibrations. I. Topology. Integrable systems and algebraic geometry (Kobe/Kyoto, 1997), 156-193, World Sci. Publishing, River Edge, NJ, 1998.

[53] M. Gross, Special Lagrangian fibrations II: Geometry. Survey in Differential Geometry, edited by S.T. Yau (1999) 341-404.

[54] M. Gross, Topological Mirror Symmetry, Invent. Math. 144 (2001), no. 1, 75-137.

[55] M. Gross and B. Siebert, Affine manifolds, log structures, and mirror symmetry. Turkish J. Math. 27 (2003), no. 1, 33-60.

[56] M. Gross and B. Siebert, Mirror symmetry via logarithmic degeneration data. I. J. Differential Geom. 72 (2006), no. 2, 169-338.

[57] M. Gross and P.M.H. Wilson, Large complex structure limits of K3 surfaces. J. Differential Geom. 55 (2000), no. 3, 475-546.

[58] S. Gukov, S.-T. Yau and E. Zaslow, Duality and fibrations on G2 manifolds. Turkish J. Math. 27 (2003), no. 1, 61-97.

[59] R. Harvey and B. Lawson, Calibrated geometries, Acta Math. 148 (1982), $47-157$.

[60] N. Hitchin, Hyper-Kahler manifolds. Seminaire Bourbaki, Vol. 1991/92. Asterisque No. 206 (1992), Exp. No. 748, 3, 137-166.

[61] N. Hitchin, The moduli space of special Lagrangian submanifolds. Dedicated to Ennio De Giorgi. Ann. Scuola Norm. Sup. Pisa Cl. Sci. (4) 25 (1997), no. 3-4, 503-515 (1998).

[62] N. Hitchin, The moduli space of complex Lagrangian submanifolds. Sir Michael Atiyah: a great mathematician of the twentieth century. Asian J. Math. 3 (1999), no. 1, 77-91.

[63] N. Hitchin, Lectures on Special Lagrangian Submanifold. Winter School on Mirror Symmetry, Vector Bundles and Lagrangian Submanifolds (Cambridge, MA, 1999), 151-182, AMS/IP Stud. Adv. Math., 23 Amer. Math. Soc., Providence, RI, 2001.

[64] N. Hitchin, The geometry of three forms in 6 and 7 dimensions, J. Differential Geom. 55 (2000), no. 3, 547-576. 
[65] N. Hitchin, Stable forms and special metrics. Global differential geometry: the mathematical legacy of Alfred Gray (Bilbao, 2000), 70-89, Contemp. Math., 288, Amer. Math. Soc., Providence, RI, 2001.

[66] K. Hori and C. Vafa, Mirror symmetry, [hep-th/0002222].

[67] K. Hori, A. Iqbal and C. Vafa, D-Branes And Mirror Symmetry [hep-th/0005247].

[68] Y. Hu and S.T. Yau, Hyperkahler manifolds and birational transformations, Adv. Theor. Math. Phys. 6 (2002), no. 3, 557-574.

[69] Y.D. Huang and N.C. Leung, A uniform description of Riemannian symmetric spaces as Grassmannians using magic square, preprint.

[70] D. Joyce, Compact Riemannian 7-manifolds with $G_{2}$-holonomy I,II, J. Diff. Geom. 43 (1996) 291 and 43 (1996) 329.

[71] D. Joyce, Compact manifolds with special holonomy, Oxford University Press, 2000.

[72] D. Joyce, On counting special Lagrangian homology 3-spheres, Topology and geometry: commemorating SISTAG, 125-151, Contemp. Math., 314, A.M.S., 2002.

[73] S. Karigiannis and N.C. Leung, Hodge theory for G_2 manifolds: Intermediate Jacobians and Abel-Jacobi maps, preprint.

[74] A. Klemm, D. Maulik, R. Pandharipande and E. Scheidegger, Noether-Lefschetz theory and the Yau-Zaslow conjecture, preprint 2008. [arXiv:0807.2477].

[75] R. Kobayashi, Ricci flow unstable cell centered at an Einstein metric on the twistor space of positive quaternion Kahler manifolds of dimension $\geq 8$. [arXiv:0801.2605].

[76] M. Kontsevich, Homological Algebra of Mirror Symmetry, Proceedings of the 1994 International Congress of Mathematicians I, Birkäuser, Zürich (1995) p. 120; [alggeom/9411018].

[77] M. Kontsevich and Y. Soibelman, Affine structures and non-Archimedean analytic spaces. The unity of mathematics, 321-385, Progr. Math., 244, Birkhauser Boston, Boston, MA, 2006.

[78] P. Kronheimer and T. Mrowka, The genus of embedded surfaces in the projective plane. Math. Res. Lett. 1 (1994), no. 6, 797-808.

[79] S.C. Lau and N.C. Leung, Conformal geometry and special holonomy, Proc. of Geometric Analysis workshop in Taiwan 2008.

[80] C. LeBrun, Einstein metrics, four-manifolds, and differential topology. Surveys in differential geometry, Vol. VIII (Boston, MA, 2002), 235-255, Surv. Differ. Geom., VIII, Int. Press, Somerville, MA, 2003.

[81] J. Lee and N.C. Leung, Yau-Zaslow formula on K3 surfaces for non-primitive classes. Geom. Topol. 9 (2005), 1977. C2012.

[82] J. Lee and N.C. Leung, Counting elliptic curves in K3 surfaces. J. Algebraic Geom. 15 (2006), 591-601.

[83] J.H. Lee and N.C. Leung, Higher dimensional knot spaces for manifolds with vector cross product, Advances in Mathematics, 213 (2007) 140-164.

[84] J.H. Lee and N.C. Leung, Geometric structures on $G_{2}$ and Spin(7)-manifolds, Adv. Theor. Math. Phys. (2009)13, no. 1, 1-31.

[85] J.H. Lee and N.C. Leung, Instantons and Branes in Manifolds with Vector Cross Product, Asian Journal of Math. 2009.

[86] Y.-P. Lee, H.-W. Lin and C.-L. Wang, Invariance of quantum rings under ordinary flops, preprint 2008.

[87] N.C. Leung, Seiberg Witten invariants and uniformizations, Mathematische Annalen, 306, (1996), 31-46.

[88] N.C. Leung, N.C. Einstein type metrics and stability on vector bundles. J. Differential Geom. 45 (1997), no. 3, 514-546.

[89] N.C. Leung, Symplectic structures on gauge theory, Comm. Math. Phys., 193, (1998), $47-67$.

[90] N.C. Leung, Non-Abelian Seiberg-Witten equations on Einstein manifolds, Journal fur die reine und angewandte Mathematik., Vol. 519, (2000), 17-29. 
[91] N.C. Leung, Topological Quantum Field Theory for Calabi-Yau threefolds and G $_{2}$ manifolds, Adv. Theor. Math. Phys. 6 (2002), no. 3, 575-591.

[92] N.C. Leung, Lagrangian submanifolds in hyperkahler manifolds and Legendre transformation. Journal of Differential Geometry (2002) Vol. 61, no.1, 107-145.

[93] N.C. Leung, Riemannian Geometry over different normed division algebras, J. Differential Geom. 61 (2002), no. 2, 289-333.

[94] N.C. Leung, Mirror symmetry without correction, Comm. Anal. Geom. 13 (2005), no. $2,287-331$.

[95] N.C. Leung, Geometry of special holonomy, ICCM 2007.

[96] N.C. Leung and C.Z. Li, Hard Lefschetz actions in Riemannian geometry with special holonomy, Math. Res. Lett. 15 (2008), no. 4, 683-698

[97] N.C. Leung and C. Vafa, Branes and toric geometry, Adv. Theor. Math. Phys. 2 (1998), 91-118.

[98] N.C. Leung and T. Wan, Hyper-Lagrangian submanifolds of Hyperkahler manifolds and Mean Curvature Flow, Journal of Geometric Analysis, vol. 17, no. 2 (2007) 343-364.

[99] N.C. Leung and X.W. Wang, Intersection theory of coassociative submanifolds in $G_{2}$-manifolds and Seiberg-Witten invariants, [Math.DG/0401419].

[100] N.C. Leung and M. Xu, Compactness of massive Seiberg-Witten equations, Asian Journal of Math., vol. 13 (2009) no.3, 359-368.

[101] N.C. Leung, S.-T. Yau and E. Zaslow, From special Lagrangian to Hermitian-YangMills via Fourier-Mukai transform. Adv. Theor. Math. Phys. (2000), Vol. 4, No. 6. [math.DG/ 0005118].

[102] N.C. Leung and J.J. Zhang, Moduli of bundles over rational surfaces and elliptic curves I: simply laced cases. Preprint.

[103] N.C. Leung and J.J. Zhang, Moduli of bundles over rational surfaces and elliptic curves II: nonsimply laced cases. Preprint.

[104] T.J. Li and A. Liu, Uniqueness of symplectic canonical class, surface cone and symplectic cone of 4-manifolds with $B^{+}=1$. J. Differential Geom. 58 (2001), no. 2, 331-370.

[105] B.H. Lian, K. Liu and S.T. Yau, Mirror principle I. Asian J. Math., vol. 1 , no. 4 (1997) 729-763.

[106] D. Matsushita, On fibre space structures of a projective irreducible symplectic manifold. Topology 38 (1999), no. 1, 79-83.

[107] D. Maulik, N. Nekrasov, A. Okounkov and R. Pandharipande. Gromov-Witten theory and Donaldson-Thomas theory, I. Compositio Math. 142(5):1263-1285, 2006.

[108] R.C. McLean, Deformation of Calibrated Submanifolds, Commun. Analy. Geom. 6 (1998) 705-747.

[109] S. Mukai, Fourier functor and its application to the moduli of bundles on an abelian variety. Algebraic geometry, Sendai, 1985, 515-550, Adv. Stud. Pure Math., 10, North-Holland, Amsterdam, 1987.

[110] K. O’Grady, A new six-dimensional irreducible symplectic variety, J. Algebraic Geom. 12 (2003), 435-505.

[111] H. Ooguri and C. Vafa, Summing up Dirichlet instantons. Phys. Rev. Lett. 77 (1996), no. $16,3296-3298$.

[112] H. Ooguri and C. Vafa, Knot invariants and topological strings. Nuclear Phys. B 577 (2000), no.3, 419-438.

[113] R. Pandharipande and R. P. Thomas, Stable pairs and BPS invariants. math.AG [arXiv:0711.3899].

[114] S. Salamon, Riemannian geometry and holonomy groups, Pitman Research Notes in Math. 201, Longman.

[115] P. Seidel, Homological mirror symmetry for the quartic surface. [math.SG/0310414]. 
[116] A. Strominger, S.-T. Yau, and E. Zaslow, Mirror Symmetry is T-Duality, Nuclear Physics B479 (1996) 243-259.

[117] C. Taubes, The existence of anti-self-dual conformal structures. J. Differential Geom. 36 (1992), no. 1, 163-253.

[118] C. Taubes, $G W=S W$ : counting curves and connections. Seiberg Witten and Gromov invariants for symplectic 4-manifolds, 275-401, First Int. Press Lect. Ser., 2, Int. Press, Somerville, MA, 2000.

[119] R. Thomas, Moment maps, monodromy and mirror manifolds, In "Symplectic geometry and mirror symmetry" , Proceedings of the 4th KIAS Annual International Conference, Seoul. Eds. K. Fukaya, Y.-G. Oh, K. Ono and G. Tian. World Scientific, 2001.

[120] R. Thomas and S.T. Yau, Special Lagrangians, stable bundles and mean curvature flow, Comm. Anal. Geom. 10 (2002), no. 5, 1075-1113.

[121] R. Thomas, appendix in Cohomology and obstructions II: curves on K-trivial threefolds. by H. Clemens. J. Algebraic Geom. 14, 705-739, 2005.

[122] G. Tian, Smoothness of the universal deformation space of compact Calabi-Yau manifolds and its Petersson-Weil metric, in Mathematical aspects of string theory, pp. 629-646, Adv. Ser. Math. Phys., 1, World Sci. Publishing, 1987.

[123] J. Tits, Algebres alternatives, algebres de Jordan et algebres de Lie exceptionnelles. Indag. Math. 28(1966), 223-237.

[124] A. Todorov, The Weil-Petersson geometry of the moduli space of $S U(n=3)$ (CalabiYau) manifolds. I. Comm. Math. Phys. 126 (1989), no. 2, 325-346.

[125] K. Uhlenbeck and S.T. Yau, On the existence of Hermitian-Yang-Mills connections in stable vector bundles, Comm. Pure Appl. Math. 39 (1986), 257-293.

[126] C. Vafa and E. Witten, Eigenvalue inequalities for fermions in gauge theories. Comm. Math. Phys. 95 (1984), no. 3, 257-276.

[127] C. Vafa and E. Witten, A strong coupling test of S-duality. Nuclear Phys. B 431 (1994), no. 1-2, 3-77.

[128] M.S. Verbitsky, Action of the Lie algebra SO(5) on the cohomology of a hyperkähler manifold, Func. Analysis and Appl. 24(2)(1990), 70-71.

[129] X.W. Wang, Balance point and stability of vector bundles over a projective manifold. Math. Res. Lett. 9 (2002), no. 2-3, 393-411.

[130] P.M.H. Wilson, The role of $c_{2}$ in Calabi-Yau classification - a preliminary survey, in Mirror Symmetry II.

[131] E. Witten, Super-symmetry on Morse theory. J. Diff. Geom. 17 (1982), 661-692.

[132] E. Witten, Topological quantum field theory. Comm. Math. Phys. 117 (1988), no. 3, $353-386$.

[133] E. Witten, Quantum field theory and the Jones polynomial. Comm. Math. Phys. 121 (1989), no. 3, 351-399.

[134] E. Witten, Mirror manifolds and topological field theory. Essays on mirror manifolds, 120-158, Int. Press, Hong Kong, 1992.

[135] E. Witten, Monopoles and four-manifolds. Math. Res. Lett. 1 (1994), no. 6, 769-796.

[136] E. Witten, Chern-Simons gauge theory as a string theory, The Floer memorial volume, 637-678, Progr. Math., 133, Birkhäuser, Basel, 1995.

[137] S.T. Yau, On the Ricci curvature of a compact Kähler manifold and the complex Monge-Ampère equation I, Comm. Pure and Appl. Math. 31, (1978) 339-411.

[138] S.T. Yau and E. Zaslow, BPS states, string duality, and nodal curves on K3. Nucl. Phys. B 471(1996) no. 3, 503-512.

The Institute of Mathematical Sciences and Department of Mathematics, The Chinese University of Hong Kong, Shatin, Hong Kong.

E-mail address: Leung@math.cuhk.edu.hk 
